\title{
Results and Analysis of
} the Research and Development Work Scope Request for Information (DE-SOL-0008246)

Brenden Heidrich

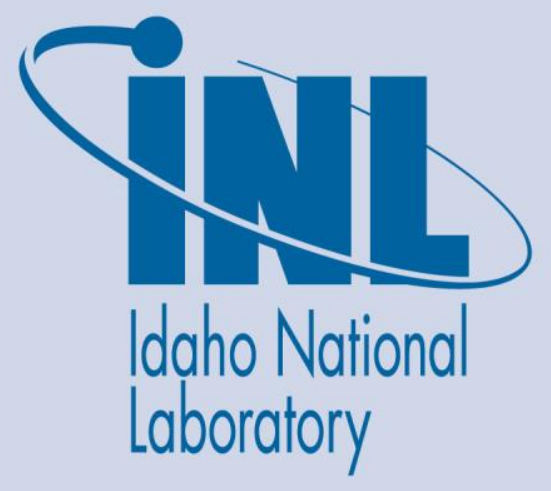

July 2015

The INL is a U.S. Department of Energy National Laboratory operated by Battelle Energy Alliance 


\section{DISCLAIMER}

This information was prepared as an account of work sponsored by an agency of the U.S. Government. Neither the U.S. Government nor any agency thereof, nor any of their employees, makes any warranty, expressed or implied, or assumes any legal liability or responsibility for the accuracy, completeness, or usefulness, of any information, apparatus, product, or process disclosed, or represents that its use would not infringe privately owned rights. References herein to any specific commercial product, process, or service by trade name, trade mark, manufacturer, or otherwise, does not necessarily constitute or imply its endorsement, recommendation, or favoring by the U.S. Government or any agency thereof. The views and opinions of authors expressed herein do not necessarily state or reflect those of the U.S. Government or any agency thereof. 
INL/EXT-15--36151

Revision 0

\title{
Results and Analysis of the Research and Development Work Scope Request for Information (DE-SOL-0008246)
}

\author{
Brenden Heidrich
}

July 2015

\author{
Idaho National Laboratory \\ Nuclear Scientific User Facilities \\ Idaho Falls, Idaho 83415
}

http://www.inl.gov

Prepared for the

U.S. Department of Energy

Office of Nuclear Energy

Under DOE Idaho Operations Office

Contract DE-AC07-05ID14517 

Nuclear Scientific User Facilities

\section{Results and Analysis of the R\&D Work Scope Request for Information (DE-SOL-0008246)}

INL/EXT-15-36151

Revision 0

July 2015

Prepared by:

Brenden Heidrich

Date

Nuclear Energy R\&D Infrastructure Lead

Approved by:

Rory Kennedy

Date

Director, NSUF 



\section{SUMMARY}

The Department of Energy (DOE) Office of Nuclear Energy (NE) released a request for information (RFI) (DE-SOL-0008246) for "University, National Laboratory, Industry and International Input to the Office of Nuclear Energy's Competitive Research and Development Work Scope Development" on April 13, 2015. DOE-NE solicited information for work scopes for the four main program areas as well as any others suggested by the community. The RFI proposal period closed on June 19, 2015.

From the 124 responses, 238 individual work scopes were extracted. Thirty-three were associated with a DOE national laboratory, including Argonne National Laboratory (ANL), Brookhaven National Laboratory (BNL), Idaho National Laboratory (INL), Los Alamos National Laboratory (LANL), Pacific Northwest National Laboratory (PNNL) and Oak Ridge National Laboratory (ORNL).

Thirty US universities submitted proposals as well as ten industrial/commercial institutions.

Four major R\&D areas emerged from the submissions, appearing in more than $15 \%$ of the proposed work scopes. These were: nuclear fuel studies, safety and risk analysis, nuclear systems analysis and design and advanced instrumentation and controls. Structural materials for nuclear power plants, used nuclear fuel disposition and various types of systems analysis were also popular, each appearing in more than $10 \%$ of the proposals.

Nuclear Energy Enabling Technologies (NEET) was the most popular program area with $42 \%$ of the proposals referencing the NEET-CTD program. The order of the remaining programs was Fuel Cycle Technologies (FC) at 34\%, Nuclear Energy Advanced Modeling and Simulation (NEAMS) at 29\% and Reactor Concepts at $17 \%$. 


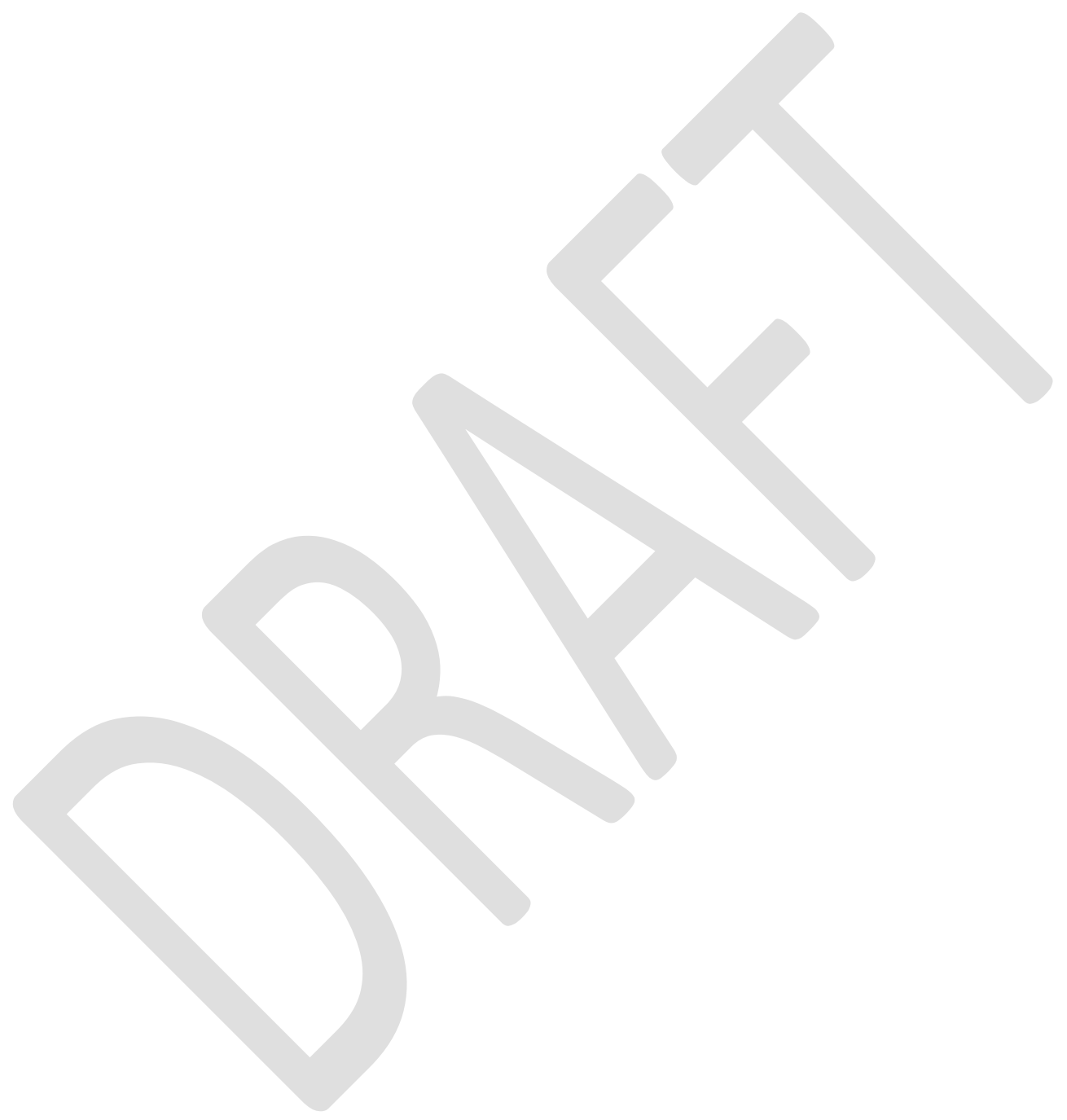




\section{CONTENTS}

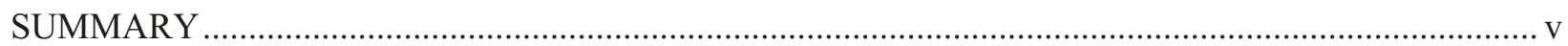

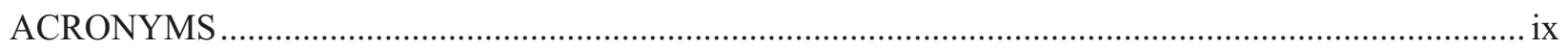

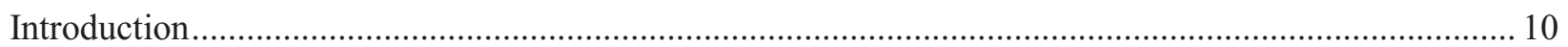

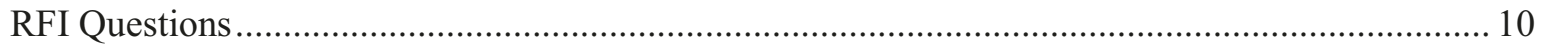

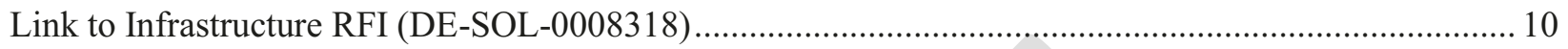

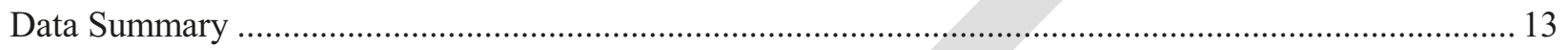

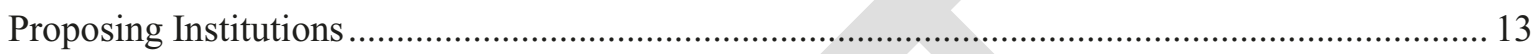

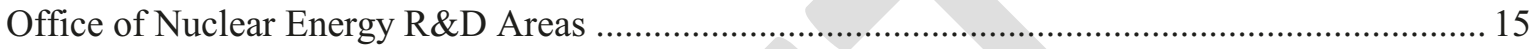

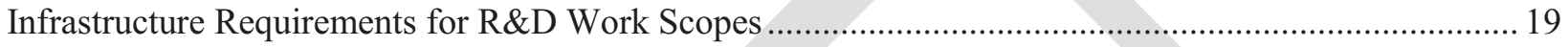

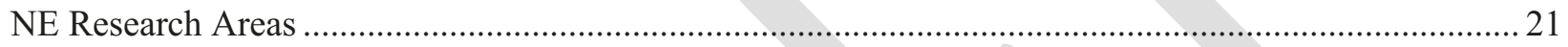

Costs Associated with Funding the Proposed Work Scopes.............................................................. 23

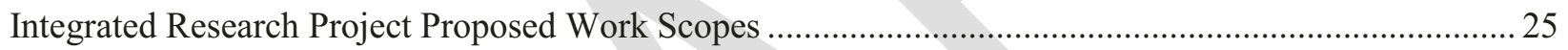

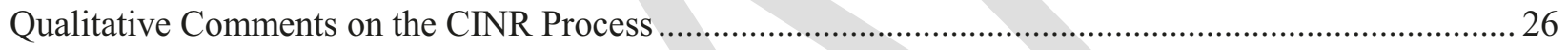

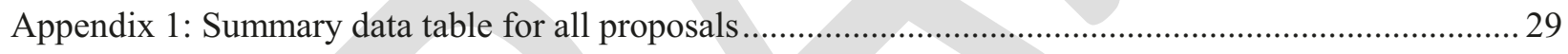

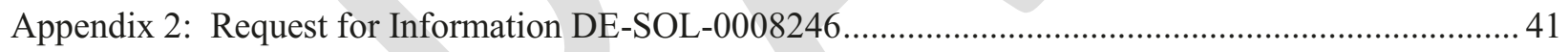




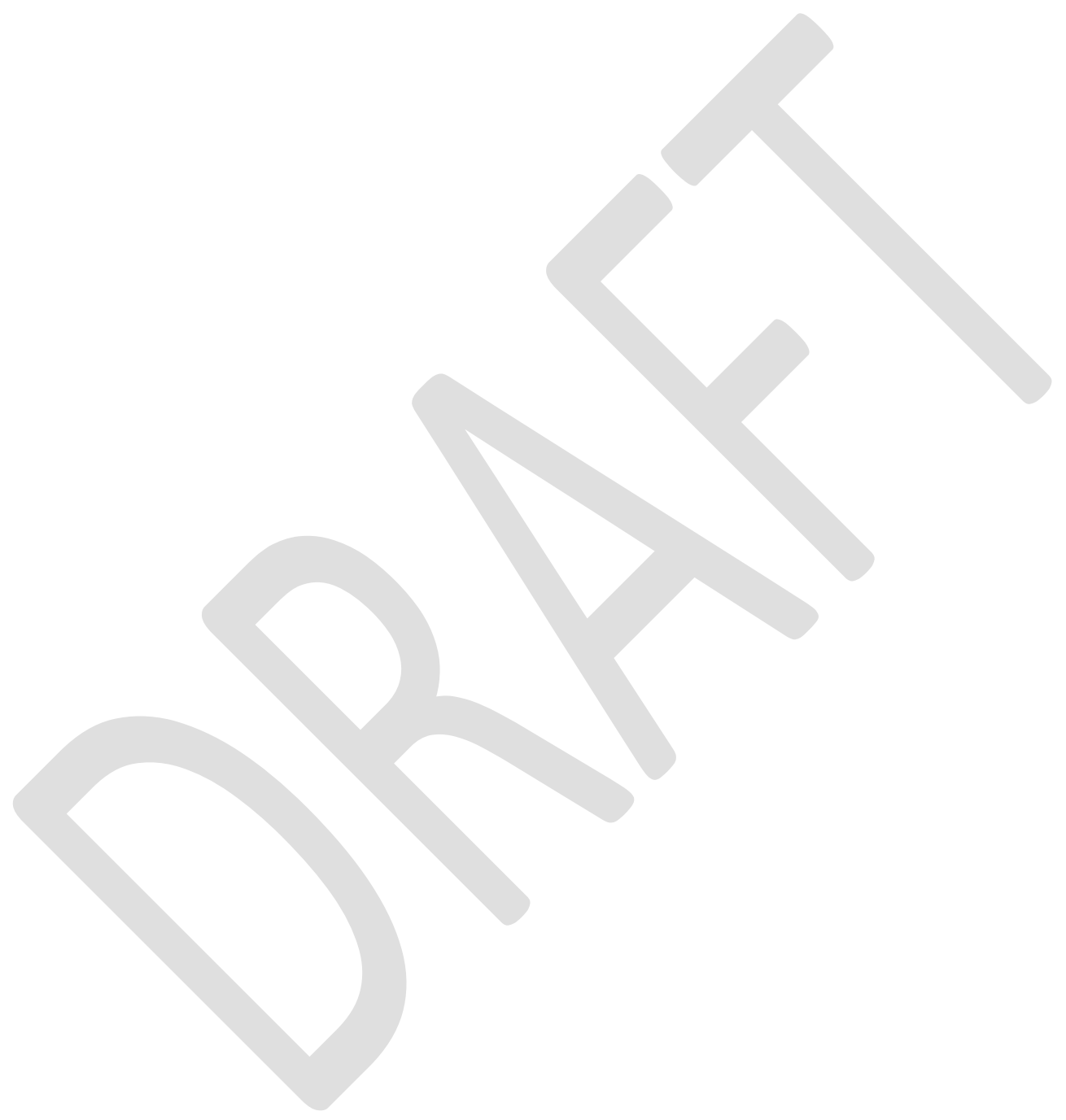




\section{ACRONYMS}

ART Advanced Reactor Technology

ATR Advanced Test Reactor

DOE Department of Energy

DRP Database Review Panel

FIMS Facility Information Management System

FY fiscal year

FC R\&D Fuel Cycle Research and Development Program

GIS Geographical Information System

IAEA International Atomic Energy Agency

IM Information Management

INL Idaho National Laboratory

LWRS Light-Water Reactor Sustainability

NE Nuclear Energy

NEAMS Nuclear Energy Advanced Modeling and Simulation

NEET-CTD Nuclear Energy Enabling Technologies - Crosscutting Technology Development

NEID Nuclear Energy Infrastructure Database

NSUF Nuclear Science User Facilities

POC point of contact

RC RD\&D Reactor Concepts Research, Development and Demonstration Program

$\mathrm{R} \& \mathrm{D}$ research and development

RFI Request for Information 


\section{Introduction}

The Department of Energy (DOE) Office of Nuclear Energy (NE) released a request for information (RFI) (DE-SOL-0008246) for "University, National Laboratory, Industry and International Input to the Office of Nuclear Energy's Competitive Research and Development Work Scope Development" on April 13, 2015. DOE-NE solicited information for work scopes for the four main program areas as well as any others suggested by the community. The RFI proposal period closed on June 19, 2015.

From the 124 responses, 238 individual work scopes were extracted. Thirty-three were associated with a DOE national laboratory, including Argonne National Laboratory (ANL), Brookhaven National Laboratory (BNL), Idaho National Laboratory (INL), Los Alamos National Laboratory (LANL), Pacific Northwest National Laboratory (PNNL) and Oak Ridge National Laboratory (ORNL). Thirty US universities submitted proposals as well as ten industrial/commercial institutions.

The four main R\&D programs are:

1. Fuel Cycle Research and Development (FC R\&D) Program

2. Reactor Concepts Research, Development and Demonstration (RC RD\&D) Program

3. Nuclear Energy Advanced Modeling and Simulation (NEAMS) Program

4. Nuclear Energy Enabling Technologies (NEET) Crosscutting Technology Development (CTD).

The RFI posed five questions to better describe the proposed work scopes. The questions are summarized below.

\section{RFI Questions}

1. Definition: Clearly define your proposed work scope, and how it relates to any part of NE's mission described in this RFI. Describe any defined goals in achieving the desired outcomes, along with appropriate metrics to assess how well those goals have been achieved.

2. Cost Estimates: What would be the estimated cost of the work scope?

3. Timeliness and Priority: Would the work scope be more focused on immediate NE program needs, or more creative, innovative and transformative?

4. Partner Requirements: Would the work scope require multiple partners?

5. Duration and Milestones: What would be a reasonable schedule duration and key milestones?

\section{Link to Infrastructure RFI (DE-SOL-0008318)}

The work scope proposals were also compared using the same infrastructure and R\&D areas utilized in the analysis of the Infrastructure RFI (DE-SOL-00008318). This allowed an extension of that analysis to include infrastructure needs not addressed in those submissions, but required to complete the research proposed in this RFI. 
1. $\quad$ R\&D Infrastructure Capability Type

The capability categories in Table 1 represent the consolidated categories from the infrastructure analysis. This data was Table 11 of INL/EXT-15-35978. Each proposed work scope was assigned to 1-3 capability categories based on expert judgement.

Table 1: Capability Categories

\begin{tabular}{|l|c|}
\hline \multicolumn{1}{|c|}{ Name } & Abbreviation \\
\hline Ion/Photon Beam Facility & IPBF \\
\hline Materials Examination & MatEx \\
\hline Reactor & MS \\
\hline Radio-chemistry Laboratory & RX \\
\hline Thermal-Hydraulic & FDF \\
\hline High-Performance Computing & RCL \\
\hline Microscope & THF \\
\hline Fuel Development & HPC \\
\hline Advanced Instrumentation & AIN \\
\hline Advanced Manufacturing & AM \\
\hline Shipping Cask (UNF) & INC \\
\hline NPP I\&C & CSK \\
\hline Concrete and Seismic Equipment & CON \\
\hline
\end{tabular}

2. Office of Nuclear Energy Mission Areas.

Each proposed work scope was assigned to 1-2 NE mission areas. Some proposals mentioned these missions; others were assigned using expert judgement.

Table 2: Office of Nuclear Energy Missions

\begin{tabular}{|c|c|l|}
\hline Number & Abbreviation & \multicolumn{1}{c|}{ Category } \\
\hline 1 & LWRS & $\begin{array}{l}\text { Improve the reliability and performance, sustain the safety and security, } \\
\text { and extend the life of current reactors by developing advanced } \\
\text { technological solutions. }\end{array}$ \\
\hline 2 & ART & $\begin{array}{l}\text { Meet the Administration's energy security and climate change goals by } \\
\text { developing technologies to support the deployment of affordable } \\
\text { advanced reactors. }\end{array}$ \\
\hline 3 & FC & $\begin{array}{l}\text { Optimize energy and waste generation, safety, and nonproliferation } \\
\text { attributes by developing sustainable fuel cycles. }\end{array}$ \\
\hline 4 & RD\&D & $\begin{array}{l}\text { Enable future nuclear energy options by developing and maintaining an } \\
\text { integrated national RD\&D framework. }\end{array}$ \\
\hline 5 & INTL & $\begin{array}{l}\text { Maintain U.S. leadership at the international level by engaging nations that } \\
\text { pursue peaceful uses of nuclear energy. }\end{array}$ \\
\hline
\end{tabular}


3. Nuclear Energy-related research areas

Table 3: Research Areas Supported by the Proposed Capability

\begin{tabular}{|c|l|}
\hline Abbreviation & \multicolumn{1}{|c|}{ Category } \\
\hline STM & Structural Materials \\
\hline NFL & Nuclear Fuels (including cladding) \\
\hline NSY & Nuclear Systems Design Studies \\
\hline PCS & Power Conversion Systems \\
\hline DRY & Dry Heat Rejection Systems \\
\hline PRO & Process Heat Transport Systems \\
\hline INC & Instrumentation and Controls \\
\hline REC & Material Recovery Processes \\
\hline WST & Waste Forms \\
\hline SST & Safeguards and Security Tech. \\
\hline UNF & Used Fuel Disposition \\
\hline RSK & Safety and Risk Assessment \\
\hline AM & Advanced Manufacturing Technologies \\
\hline SYS & Systems Analysis \\
\hline SDP & Space and Defense Power Systems \\
\hline CON & Concrete and Seismic Studies \\
\hline
\end{tabular}

4. Respondent Type

Each respondent to the RFI was placed in one of the following categories, based on their type of organization.

Table 4: Capability Location Categories

\begin{tabular}{|l|l|}
\hline \multicolumn{1}{|c|}{ Category } & \multicolumn{1}{c|}{ Definition } \\
\hline University & A US academic institution of higher learning. \\
\hline National Laboratory & A government-owned contractor-operated entity. \\
\hline Industry & $\begin{array}{l}\text { An entity that is not a University or National } \\
\text { Laboratory. This can be a for-profit entity, like a utility } \\
\text { or a vendor, or a not-for-profit entity, like EPRI. }\end{array}$ \\
\hline
\end{tabular}




\section{Data Summary}

The RFI proposal period closed on June 19, 2015. At this point, 46 institutions had submitted complete responses. The quality of the responses varied, with the majority of proposers adhering to the suggested format supplied in the RFI. Some of the requested cost data was missing, with only 198 of 238 respondents supplying both cost estimates and planned durations (83\%).

From the 124 responses, 238 individual work scopes were extracted.

\section{Proposing Institutions}

Forty-Six institutions proposed work scopes through this RFI. Thirty-three were associated with a DOE national laboratory, including Argonne National Laboratory (ANL), Brookhaven National Laboratory (BNL), Idaho National Laboratory (INL), Los Alamos National Laboratory (LANL), Pacific Northwest National Laboratory (PNNL) and Oak Ridge National Laboratory (ORNL). Thirty US universities submitted proposals as well as ten industrial/commercial institutions. The raw distributions are shown in Table 5 and Figure 1.

Table 5: Proposing Institution Types

\begin{tabular}{|c|c|c|}
\hline Institution Type & Count & Frequency \\
\hline Industry & 62 & $26 \%$ \\
\hline National Laboratory & 70 & $29 \%$ \\
\hline University & 106 & $45 \%$ \\
\hline Total & 238 & $100 \%$ \\
\hline
\end{tabular}

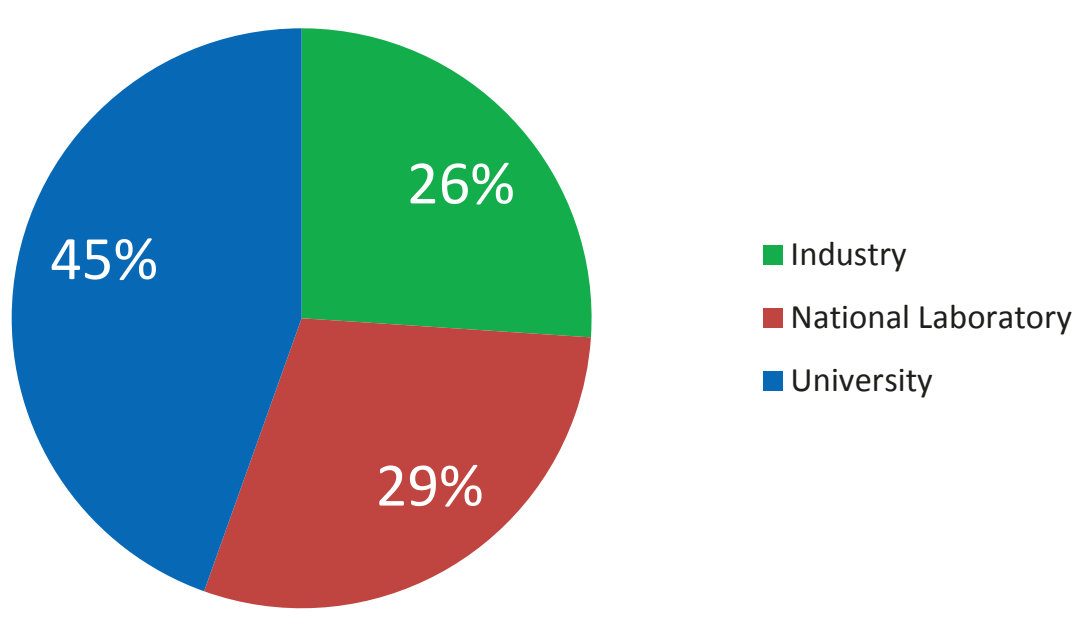

Figure 1: Distribution of Proposing Institutions to Work Scope RFI 
Many of the proposing institutions suggested multiple work scopes. Some of this was a result of multiple proposals and some were multiple work scopes within a single proposal. Table 6 shows the top 20 institutions. Figure 2 shows the distribution of institutions.

Table 6: Top 20 Institutions with most Work Scope Proposals

\begin{tabular}{|l|c|}
\hline \multicolumn{1}{|c|}{ Institution } & total work scopes \\
\hline Argonne National Laboratory & 24 \\
\hline GE Hitachi & 20 \\
\hline AREVA Federal Services & 17 \\
\hline Pacific Northwest National Laboratory & 13 \\
\hline Oak Ridge National Laboratory & 12 \\
\hline University of California, Berkeley & 12 \\
\hline NuScale Power & 11 \\
\hline Idaho National Laboratory & 11 \\
\hline Pennsylvania State University & 9 \\
\hline University of Michigan & 8 \\
\hline University of Illinois, Urbana Champaign & 8 \\
\hline Brookhaven National Laboratory & 7 \\
\hline North Carolina State University & 7 \\
\hline Westinghouse Electric Company LLC & 6 \\
\hline Rensselaer Polytechnic Institute & 6 \\
\hline Texas A\&M University & 6 \\
\hline Louisiana State University & 5 \\
\hline Purdue University & 5 \\
\hline University of Pittsburgh & 5 \\
\hline Kansas State University & 5 \\
\hline
\end{tabular}




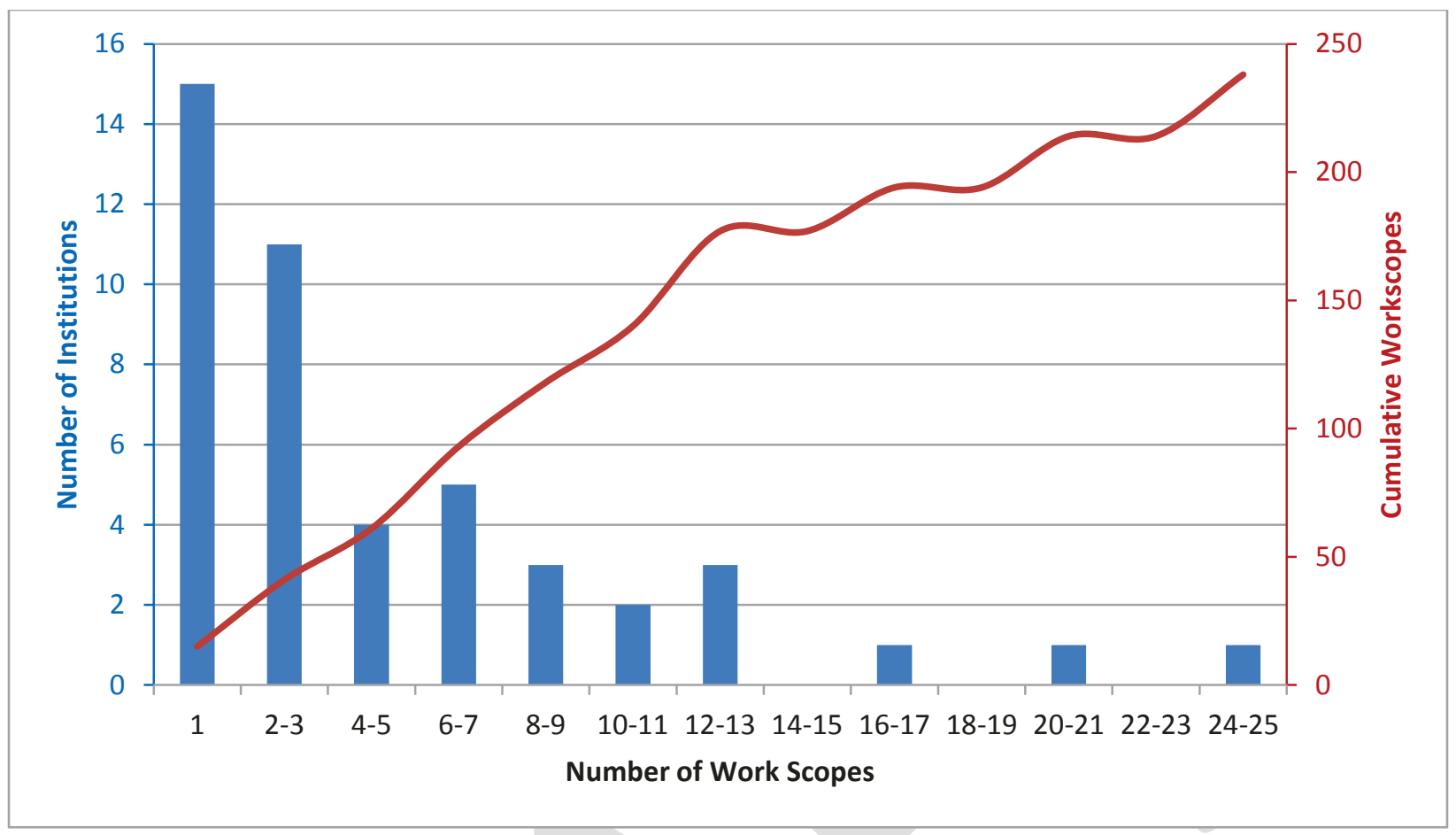

Figure 2: Distribution of Work Scope Proposals and Institutions

\section{Office of Nuclear Energy R\&D Areas}

The proposer was able to state particular NE R\&D areas as well as programs to which the proposed work scope would apply. Both a primary and a secondary choice could be made. Not all proposers made a secondary choice. Table 7 is a summary of the data. Figures 3,4 and 5 show the distribution of the primary, secondary and aggregate choices. Figure 6 shows the probability that a given R\&D area would be in any given proposal. NEET-CTD is the most common primary and secondary choice by proposers.

Table 7: NE R\&D Area Data Summary

\begin{tabular}{|l|c|c|c|c|c|c|c|}
\hline Program & $\begin{array}{c}\text { NE } \\
\text { Primary }\end{array}$ & $\begin{array}{c}\text { NE } \\
\text { Secondary }\end{array}$ & Total & $\begin{array}{c}\text { Primary } \\
\text { Frequency }\end{array}$ & $\begin{array}{c}\text { Secondary } \\
\text { Frequency }\end{array}$ & $\begin{array}{c}\text { Total } \\
\text { Frequency }\end{array}$ & $\begin{array}{c}\% \text { in any } \\
\text { proposal }\end{array}$ \\
\hline NEET-CTD & 81 & 18 & 99 & $34 \%$ & $35 \%$ & $34 \%$ & $42 \%$ \\
\hline FC R\&D & 70 & 10 & 80 & $29 \%$ & $20 \%$ & $28 \%$ & $34 \%$ \\
\hline NEAMS & 64 & 6 & 70 & $27 \%$ & $12 \%$ & $24 \%$ & $29 \%$ \\
\hline RC RD\&D & 23 & 17 & 40 & $10 \%$ & $33 \%$ & $14 \%$ & $17 \%$ \\
\hline
\end{tabular}




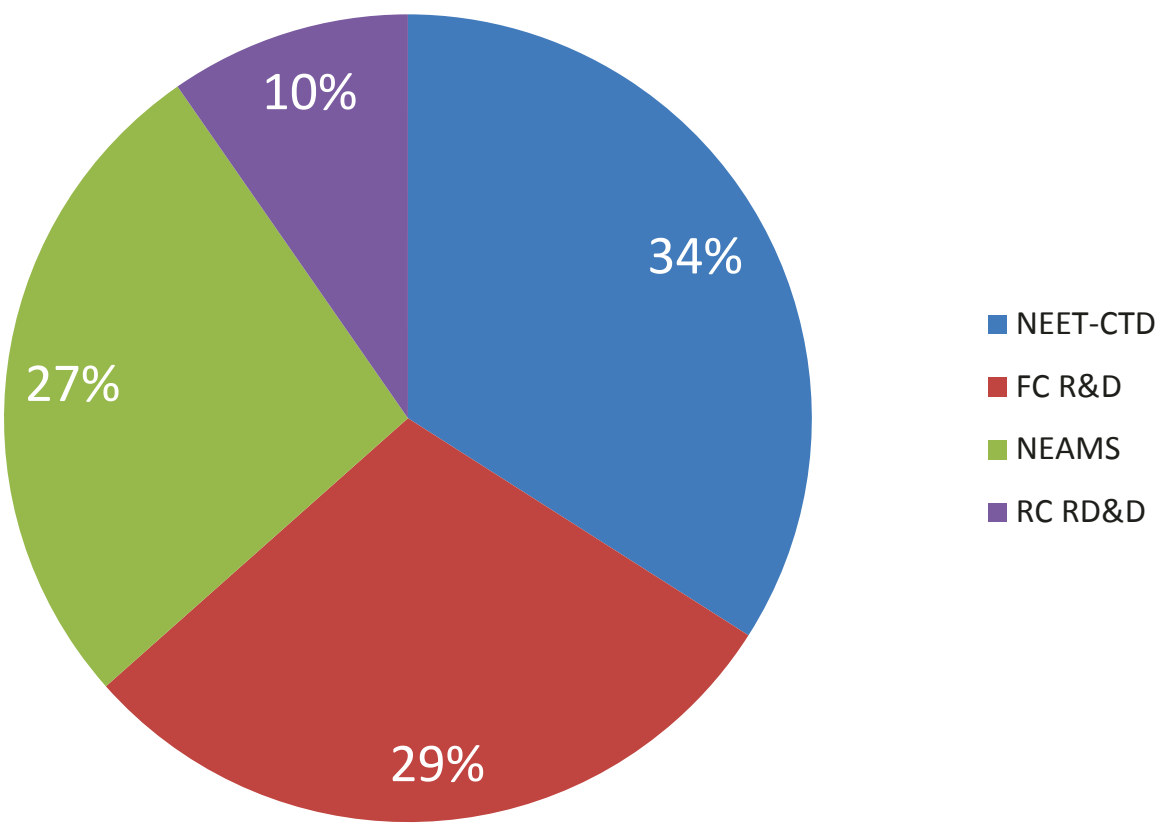

Figure 3: Primary Mission Frequency

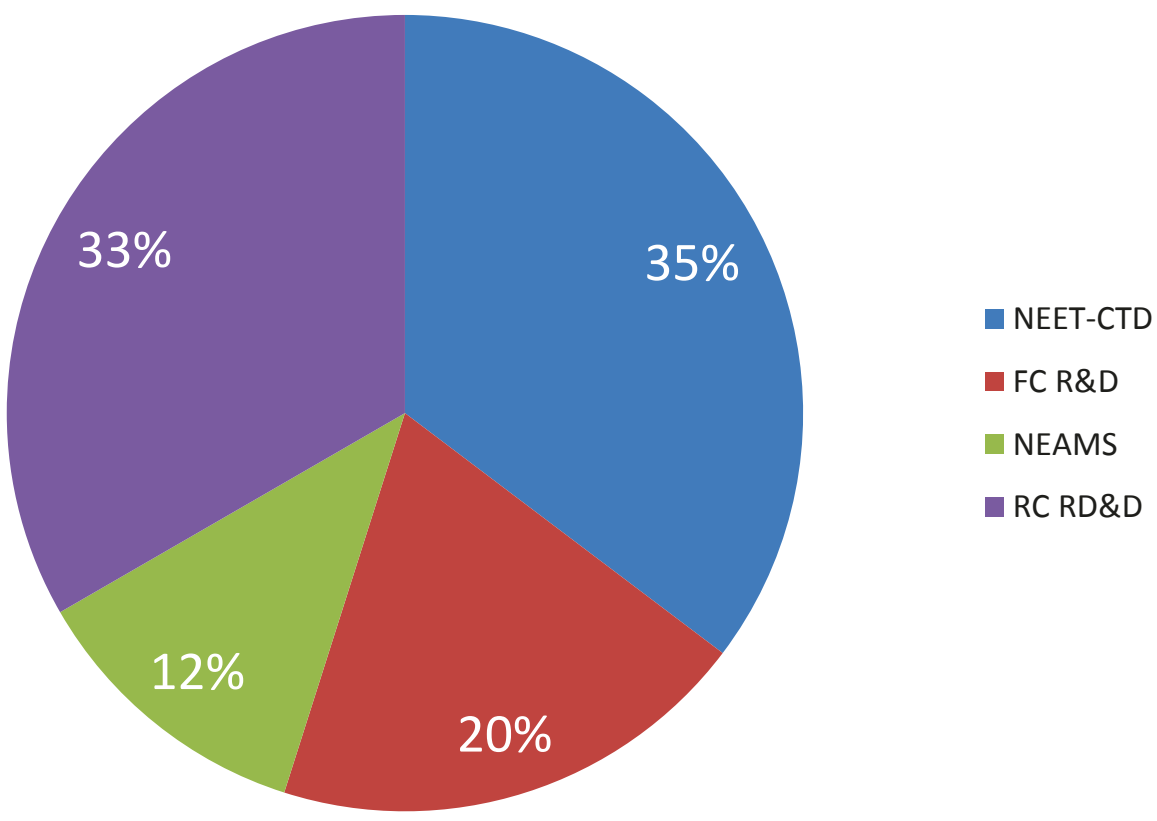

Figure 4: Secondary Mission Frequency 


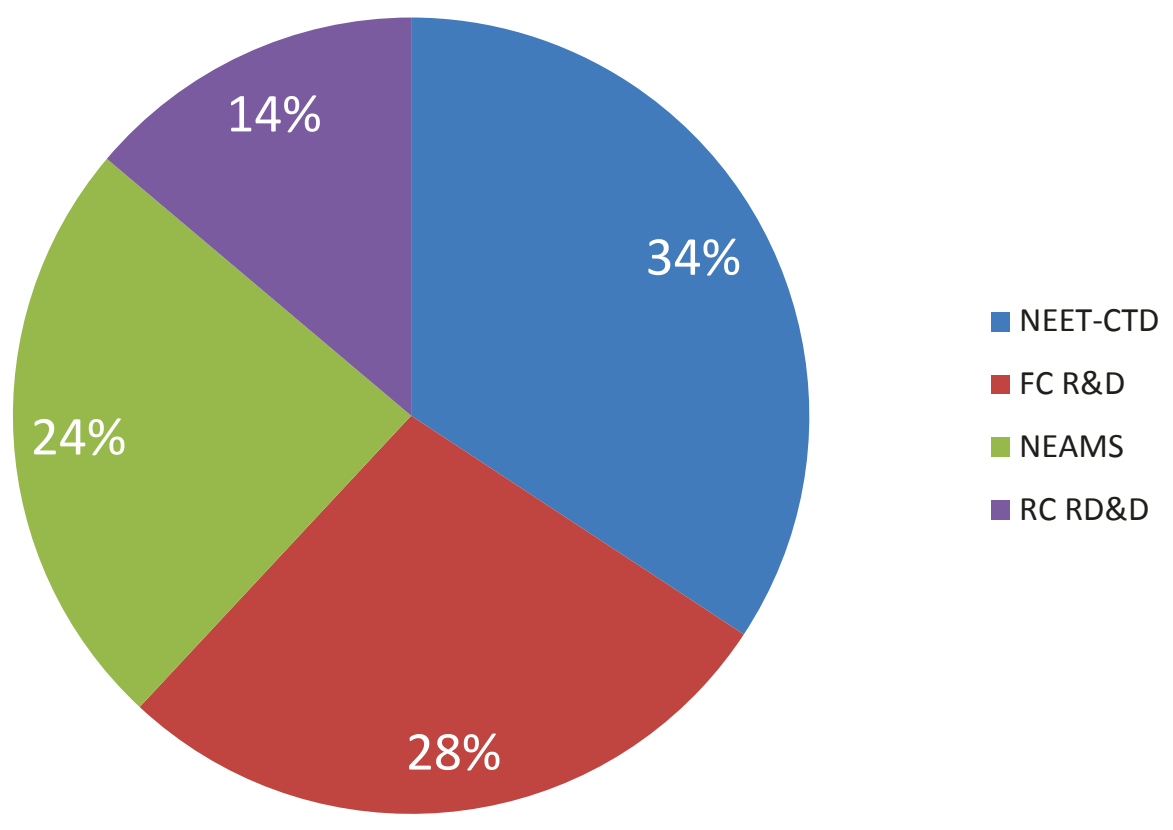

Figure 5: Total R\&D Area Frequency

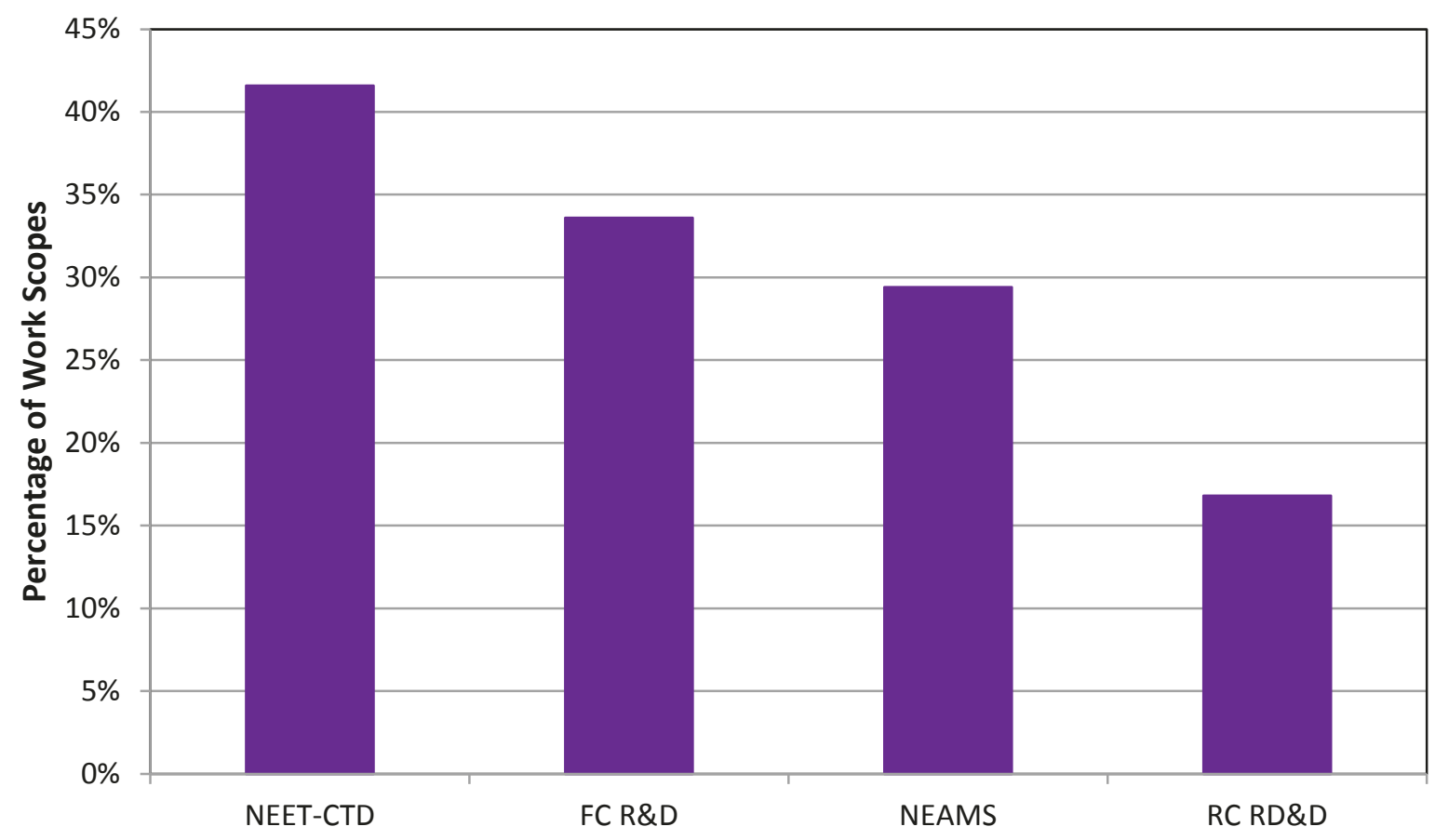

Figure 6: Percentage of Work scopes within each NE Mission Area 
The program areas where work scopes were proposed were correlated to the type of institution proposing the work. Table 8 and Figure 7 show the distribution of proposing institutions by program area. Universities were most likely to propose into the NEAMS area, likely because the infrastructure requirements for computer simulation are very small. Industry proposed most often into the Reactor Concepts area, likely in support of the LWRS program. National Laboratories were less skewed in their behavior, but were predisposed to the NEET-CTD program area, likely to support the multi-mission capabilities of the laboratories.

Table 8: NE Program Area by Institution Type

\begin{tabular}{|c|c|c|c|c|}
\hline Institution Type & NEAMS & FC R\&D & NEET-CTD & RC RD\&D \\
\hline Industry & $18 \%$ & $32 \%$ & $24 \%$ & $50 \%$ \\
\hline National Laboratory & $32 \%$ & $25 \%$ & $45 \%$ & $28 \%$ \\
\hline University & $50 \%$ & $42 \%$ & $31 \%$ & $22 \%$ \\
\hline Total & $100 \%$ & $100 \%$ & $100 \%$ & $100 \%$ \\
\hline
\end{tabular}

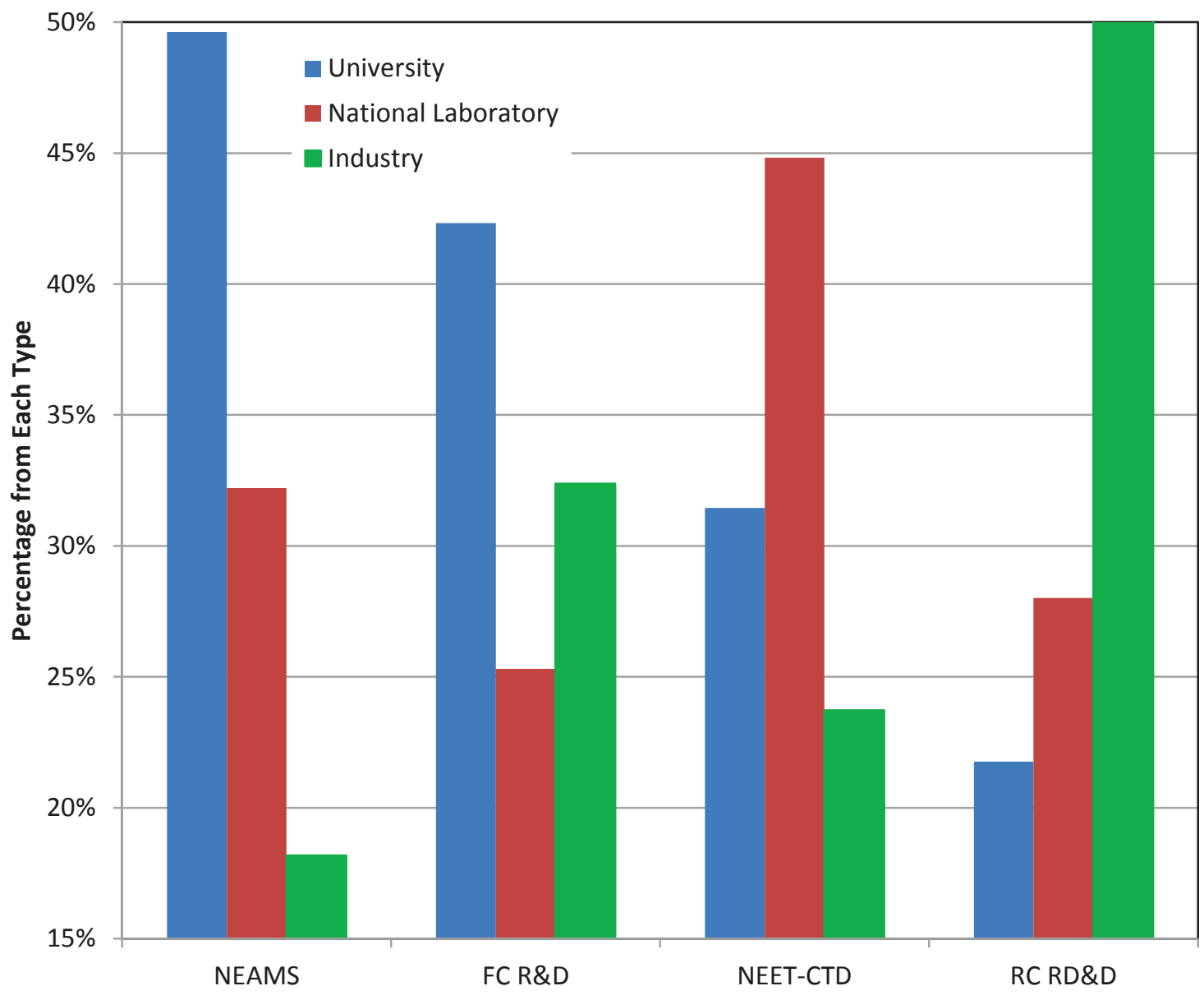

Figure 7: Program Application Normalized to Participation Level 


\section{Infrastructure Requirements for R\&D Work Scopes}

This RFI was focused on work scopes for research and development work, but that work often requires significant infrastructure capabilities. Each proposed work scope was linked to one or more of the combined infrastructure categories from the Infrastructure RFI analysis. Thirty-two of the 238 proposals did not require associated infrastructure. These were mostly in the systems analysis, safety and risk and nuclear systems design studies R\&D areas (71\%).

Tables 9 and 10 and Figure 8 show the distribution of infrastructure required for the proposed work scopes. Up to three infrastructure areas could be attached to a proposal and two R\&D areas. All of this data was aggregated so that any of the infrastructure areas were linked to all of the R\&D areas. Because of this technique, some of the correlations may not be very strong.

Table 9 shows the important infrastructure requirements for each R\&D area. The data has been converted to a percent of all infrastructure associated with each of the R\&D work scope proposals. The data is summed across the rows (work scopes). Most of the R\&D areas have strong preferences for one or two infrastructure types.

Table 9: Important Infrastructure Requirements for Each Proposed R\&D Area

\begin{tabular}{|c|c|c|c|c|c|c|c|c|c|c|c|c|c|}
\hline \multicolumn{14}{|c|}{ Infrastructure Areas } \\
\hline R\&D Areas & AIN & AM & CON & CSK & FDF & HPC & IGBF & INC & MatEx & $\mathrm{RCL}$ & RX & THF & Total \\
\hline AM & $17 \%$ & $48 \%$ & 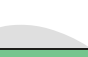 & & $7 \%$ & & $3 \%$ & $3 \%$ & $14 \%$ & $3 \%$ & & $3 \%$ & $100 \%$ \\
\hline CON & & $7 \%$ & $43 \%$ & & & & & 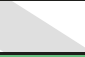 & $50 \%$ & & & & $100 \%$ \\
\hline INC & $46 \%$ & $5 \%$ & & & $2 \%$ & & 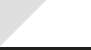 & $43 \%$ & & & $2 \%$ & $3 \%$ & $100 \%$ \\
\hline NFL & $4 \%$ & $4 \%$ & & $1 \%$ & $22 \%$ & $7 \%$ & $7 \%$ & $1 \%$ & $26 \%$ & $7 \%$ & $18 \%$ & $4 \%$ & $100 \%$ \\
\hline NSY & $6 \%$ & & & & $3 \%$ & $24 \%$ & $3 \%$ & $5 \%$ & & $2 \%$ & $11 \%$ & $46 \%$ & $100 \%$ \\
\hline PCS & & 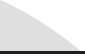 & 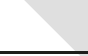 & $50 \%$ & + & + & ) & & & & & $50 \%$ & $100 \%$ \\
\hline PRO & $8 \%$ & $8 \%$ & & & & 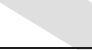 & & $8 \%$ & & $8 \%$ & & $69 \%$ & $100 \%$ \\
\hline REC & & & & 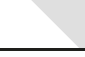 & 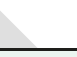 & & & & & $100 \%$ & & & $100 \%$ \\
\hline RSK & $15 \%$ & & $6 \%$ & $1 \%$ & $3 \%$ & $16 \%$ & $2 \%$ & $20 \%$ & $11 \%$ & $6 \%$ & $4 \%$ & $16 \%$ & $100 \%$ \\
\hline SDP & 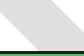 & $50 \%$ & & & $50 \%$ & & & & & & & & $100 \%$ \\
\hline SST & $50 \%$ & 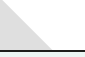 & & & $33 \%$ & & & $17 \%$ & & & & & $100 \%$ \\
\hline STM & $5 \%$ & $7 \%$ & & ( & & $2 \%$ & $14 \%$ & & $47 \%$ & $14 \%$ & $12 \%$ & & $100 \%$ \\
\hline SYS & $21 \%$ & 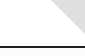 & & $14 \%$ & & $21 \%$ & & $14 \%$ & $7 \%$ & $7 \%$ & $7 \%$ & $7 \%$ & $100 \%$ \\
\hline UNF & $3 \%$ & $3 \%$ & V & $22 \%$ & $27 \%$ & & & & $8 \%$ & $30 \%$ & & $8 \%$ & $100 \%$ \\
\hline WST & & $10 \%$ & & & $30 \%$ & & $3 \%$ & & $10 \%$ & $37 \%$ & $3 \%$ & $7 \%$ & $100 \%$ \\
\hline
\end{tabular}

Table 10 is based on the same data, analyzed to show the R\&D areas that are supported by a particular type of infrastructure. The data is summed over the columns. Once again, there are specific R\&D areas that a type of infrastructure will support. 
Table 10: Infrastructure Types that Support Various R\&D Areas

\begin{tabular}{|c|c|c|c|c|c|c|c|c|c|c|c|c|}
\hline \multirow[b]{2}{*}{ R\&D Areas } & \multicolumn{12}{|c|}{ Infrastructure Areas } \\
\hline & AIN & AM & CON & CSK & FDF & HPC & IGBF & INC & MatEx & RCL & $\mathbf{R X}$ & THF \\
\hline AM & $8 \%$ & $45 \%$ & & & $4 \%$ & & $5 \%$ & $2 \%$ & $5 \%$ & $2 \%$ & & $2 \%$ \\
\hline CON & & $3 \%$ & $55 \%$ & & & & & & $9 \%$ & & & \\
\hline INC & $44 \%$ & $10 \%$ & & & $2 \%$ & & & $49 \%$ & & & $3 \%$ & $3 \%$ \\
\hline NFL & $6 \%$ & $13 \%$ & & $8 \%$ & $42 \%$ & $18 \%$ & $37 \%$ & $2 \%$ & $36 \%$ & $16 \%$ & $49 \%$ & $6 \%$ \\
\hline NSY & $6 \%$ & & & & $4 \%$ & $38 \%$ & $11 \%$ & $6 \%$ & & $2 \%$ & $19 \%$ & $44 \%$ \\
\hline PCS & & & & $8 \%$ & & & 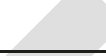 & & & & & $2 \%$ \\
\hline PRO & $2 \%$ & $3 \%$ & & & & & 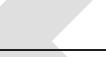 & $2 \%$ & & $2 \%$ & & $14 \%$ \\
\hline REC & & & & & & 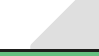 & 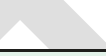 & & & $2 \%$ & & \\
\hline RSK & $20 \%$ & & $45 \%$ & $8 \%$ & $6 \%$ & $35 \%$ & $11 \%$ & $34 \%$ & $13 \%$ & $11 \%$ & $11 \%$ & $21 \%$ \\
\hline SDP & & $3 \%$ & & & $2 \%$ & 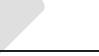 & & 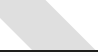 & & & & \\
\hline SST & $5 \%$ & & & & $4 \%$ & & & $2 \%$ & & & & \\
\hline STM & $3 \%$ & $10 \%$ & & & 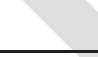 & $3 \%$ & $32 \%$ & & $27 \%$ & $13 \%$ & $14 \%$ & \\
\hline SYS & $5 \%$ & & & $15 \%$ & & $8 \%$ & & $4 \%$ & $1 \%$ & $2 \%$ & $3 \%$ & $2 \%$ \\
\hline UNF & $2 \%$ & $3 \%$ & & $62 \%$ & $19 \%$ & 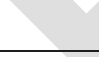 & 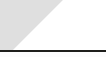 & & $4 \%$ & $24 \%$ & & $5 \%$ \\
\hline WST & & $10 \%$ & & 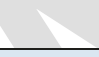 & $17 \%$ & & $5 \%$ & & $4 \%$ & $24 \%$ & $3 \%$ & $3 \%$ \\
\hline Grand Total & $100 \%$ & $100 \%$ & $100 \%$ & $100 \%$ & $100 \%$ & $100 \%$ & $100 \%$ & $100 \%$ & $100 \%$ & $100 \%$ & $100 \%$ & $100 \%$ \\
\hline
\end{tabular}

Finally, Figure 8 displays the probability that a given type of infrastructure was required for any given proposed work scope. This is the relative popularity of each type of infrastructure in this RFI.

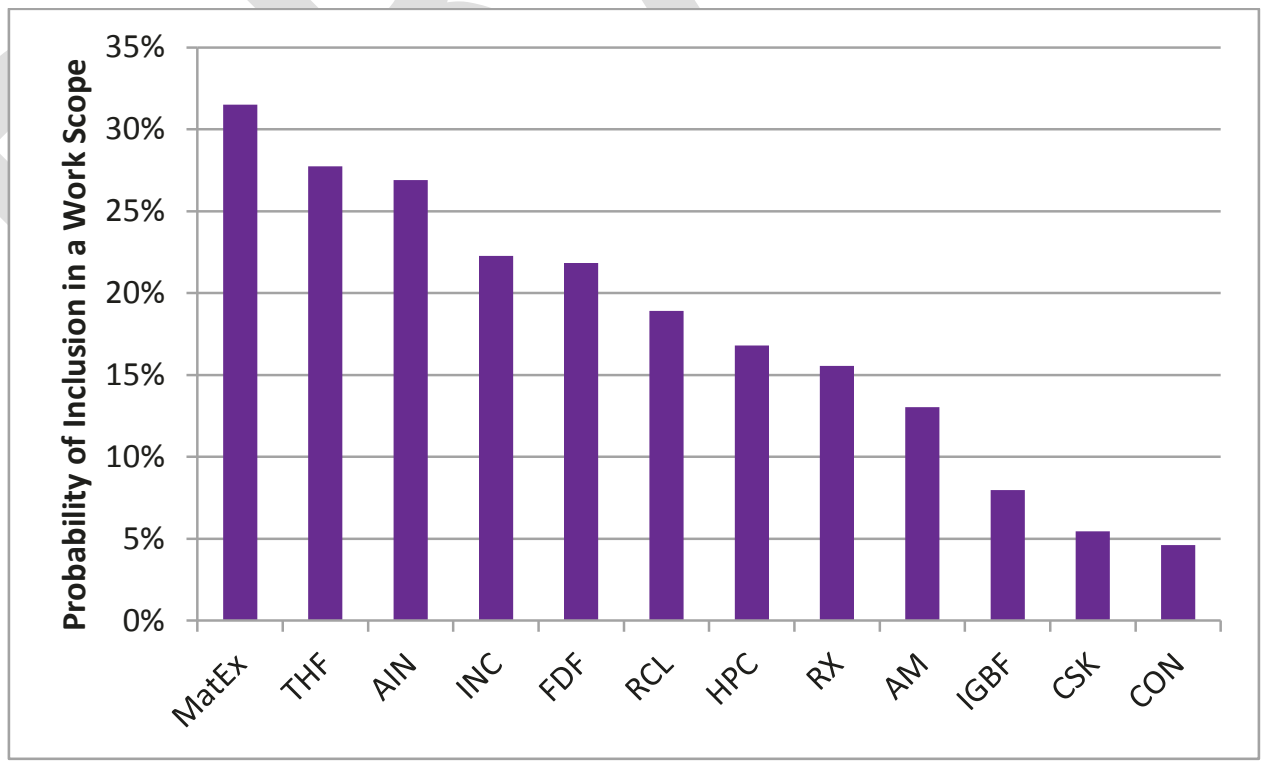

Figure 8: Probability of requiring a given Type of Infrastructure 


\section{NE Research Areas}

Each proposed work scope was matched to a set of research areas originally used for the Infrastructure RFI. Up to two areas could be assigned to a given proposed work scope. Table 11 and Figure 9 show the distribution of these research areas. Figure 10 shows the probability of a given research area appearing in any given work scope proposal.

Table 11: Frequency of Research Areas in the set of Proposed Work Scopes

\begin{tabular}{|l|c|c|c|}
\hline \multicolumn{1}{|c|}{ Research Area } & Abbreviation & TOTAL & Frequency \\
\hline Nuclear Fuels (including cladding) & NFL & 72 & $19 \%$ \\
\hline Safety and Risk Assessment & RSK & 72 & $19 \%$ \\
\hline Nuclear Systems Design Studies & NSY & 57 & $15 \%$ \\
\hline Instrumentation and Controls & INC & 36 & $10 \%$ \\
\hline Structural Materials & STM & 28 & $7 \%$ \\
\hline Used Fuel Disposition & UNF & 28 & $7 \%$ \\
\hline Systems Analysis & SYS & 24 & $6 \%$ \\
\hline Waste Forms & WST & 19 & $5 \%$ \\
\hline Advanced Manufacturing Technologies & AM & 15 & $4 \%$ \\
\hline Process Heat Transport Systems & PRO & 11 & $3 \%$ \\
\hline Concrete and Seismic & CON & 7 & $2 \%$ \\
\hline Safeguards and Security Tech. & SST & 4 & $1 \%$ \\
\hline Power Conversion Systems & PCS & 2 & $1 \%$ \\
\hline Space and Defense Power Systems & SDP & 1 & $0 \%$ \\
\hline Dry Heat Rejection Systems & DRY & 0 & $0 \%$ \\
\hline Material Recovery Processes & REC & 0 & $0 \%$ \\
\hline
\end{tabular}



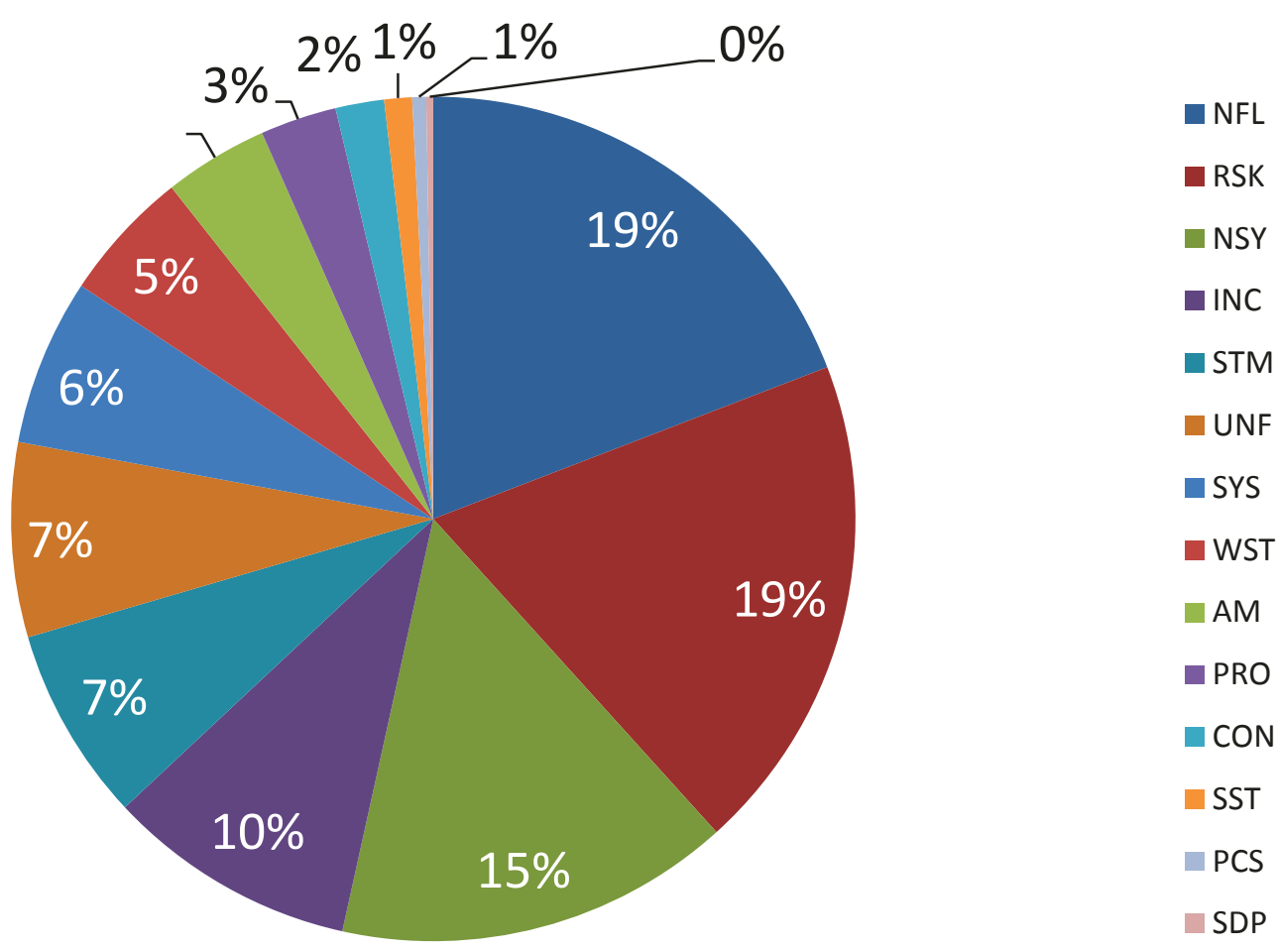

Figure 9: Distribution of NE R\&D Areas listed in RFI Reponses

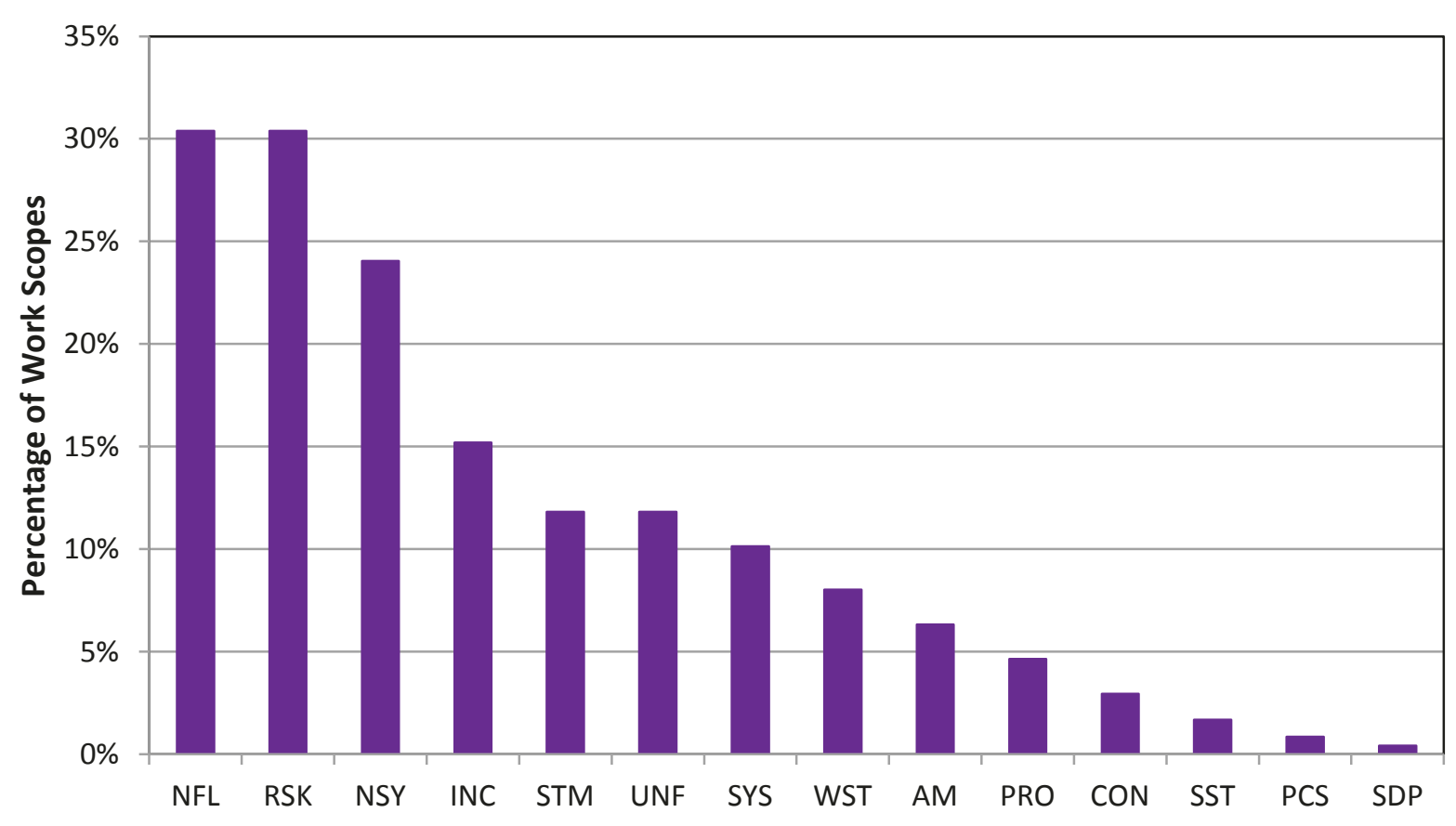

Figure 10: Percentage of Proposals that Included each NE R\&D Area 
While the distribution of research areas is clear, the relationship among them is also important. Two choices were allowed for each proposal. The selection of the first area had a strong influence on the second choice. Figure 11 shows the relationship among the research areas with respect to first and second choices. Safety and Risk Analysis is the most probable secondary choice of research areas. This may be real, or it may be a function of the proposer adding a statement about safety in order to provide gravitas to the proposal.

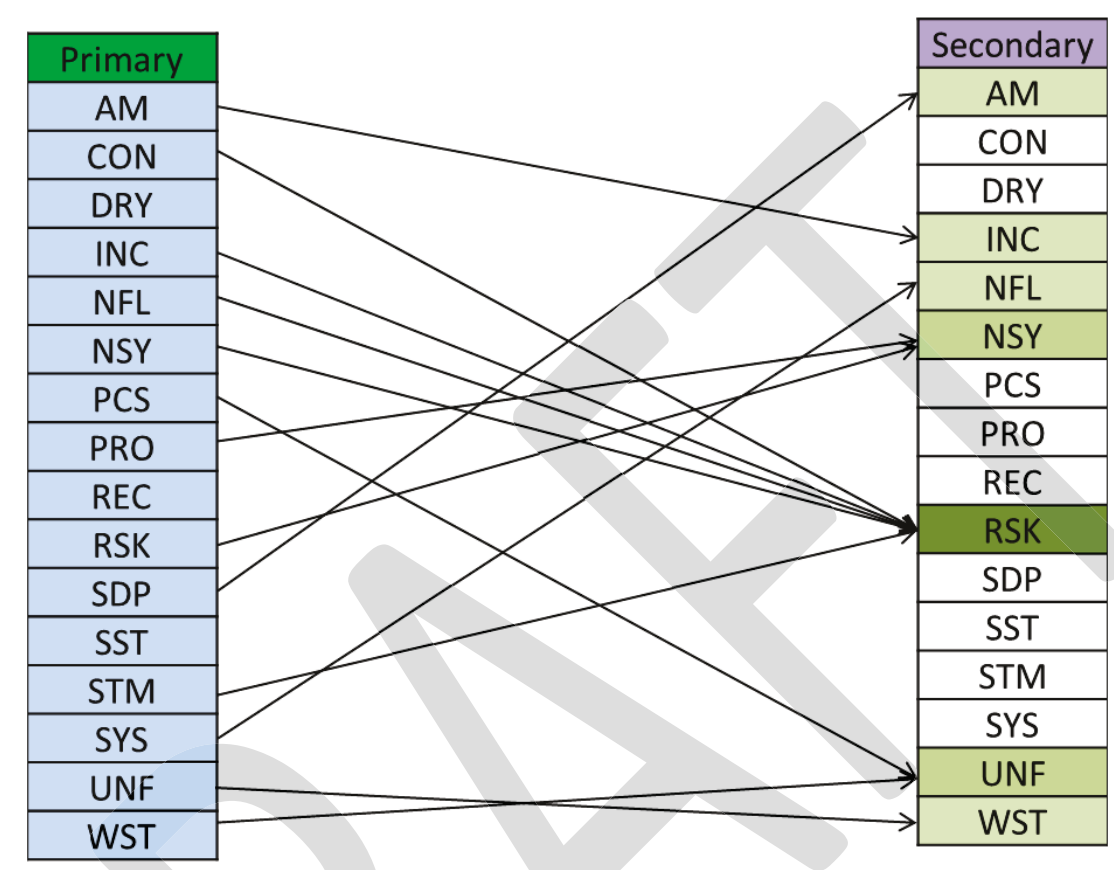

Figure 11: Preferred Secondary Research Area Choices in set of Proposed Work Scopes

\section{Costs Associated with Funding the Proposed Work Scopes}

The respondents were asked to provide a cost and schedule estimate for the proposed work scopes. Figures 12, 13 and 14 show the distribution of total costs, expected schedule duration and the associated cost per year for each work scope. Most of the proposals provided an estimate of $\$ 800,000$ and a three-year duration, which has been the typical cost and length. The distributions were as expected, with a few high-cost and long-term proposals. 


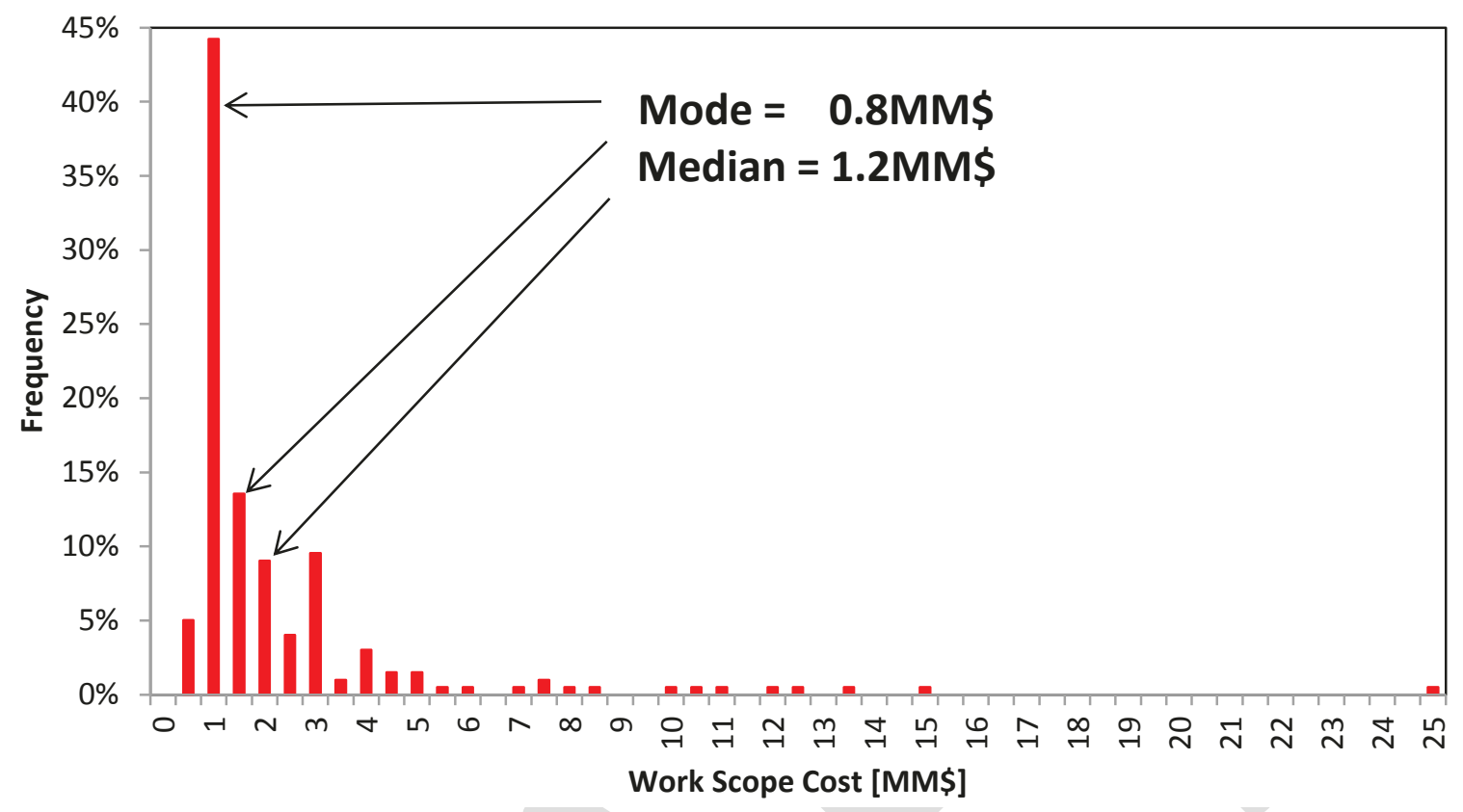

Figure 12: Total Work Scope Cost

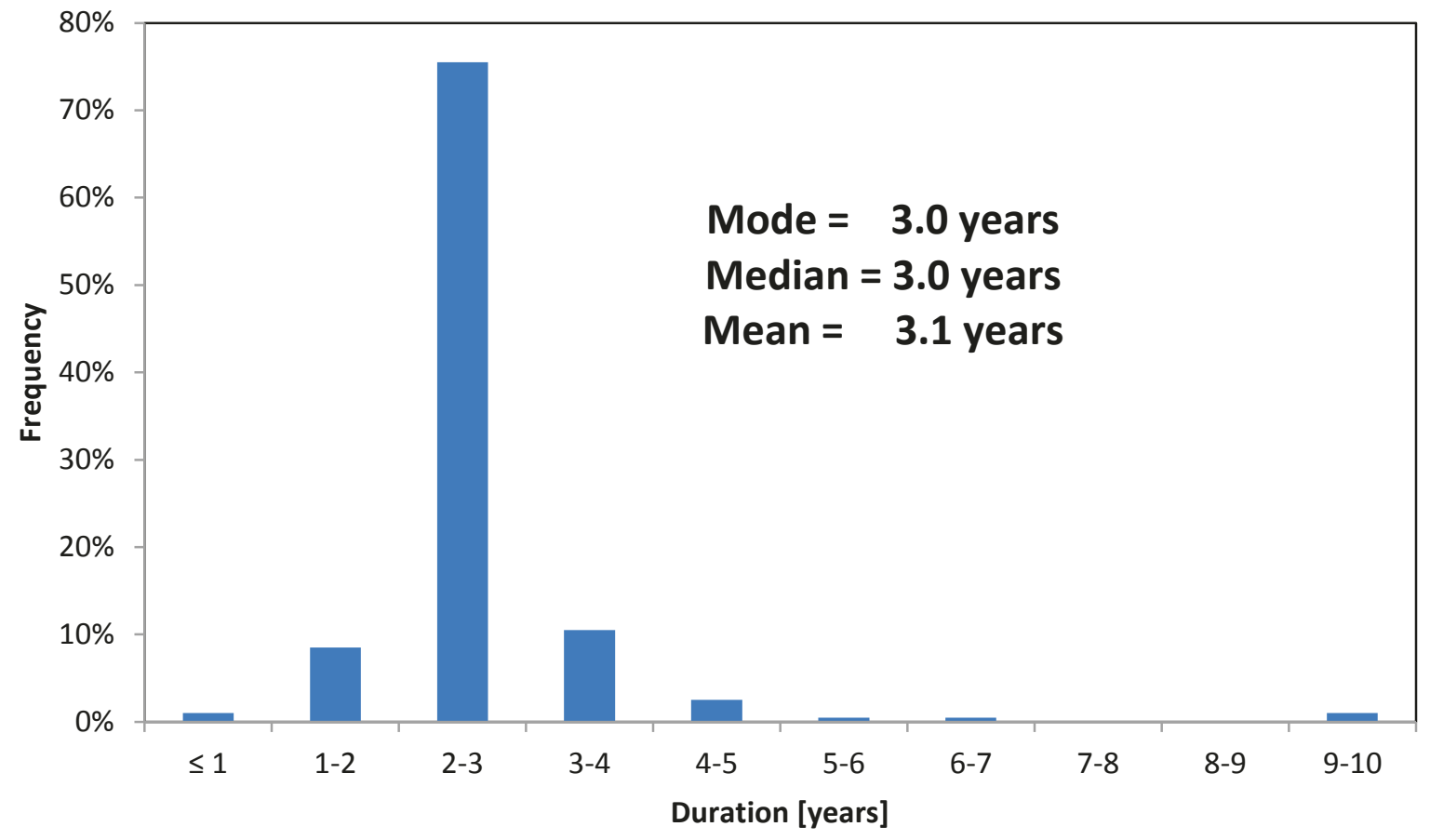

Figure 13: Expected Work Scope Duration 


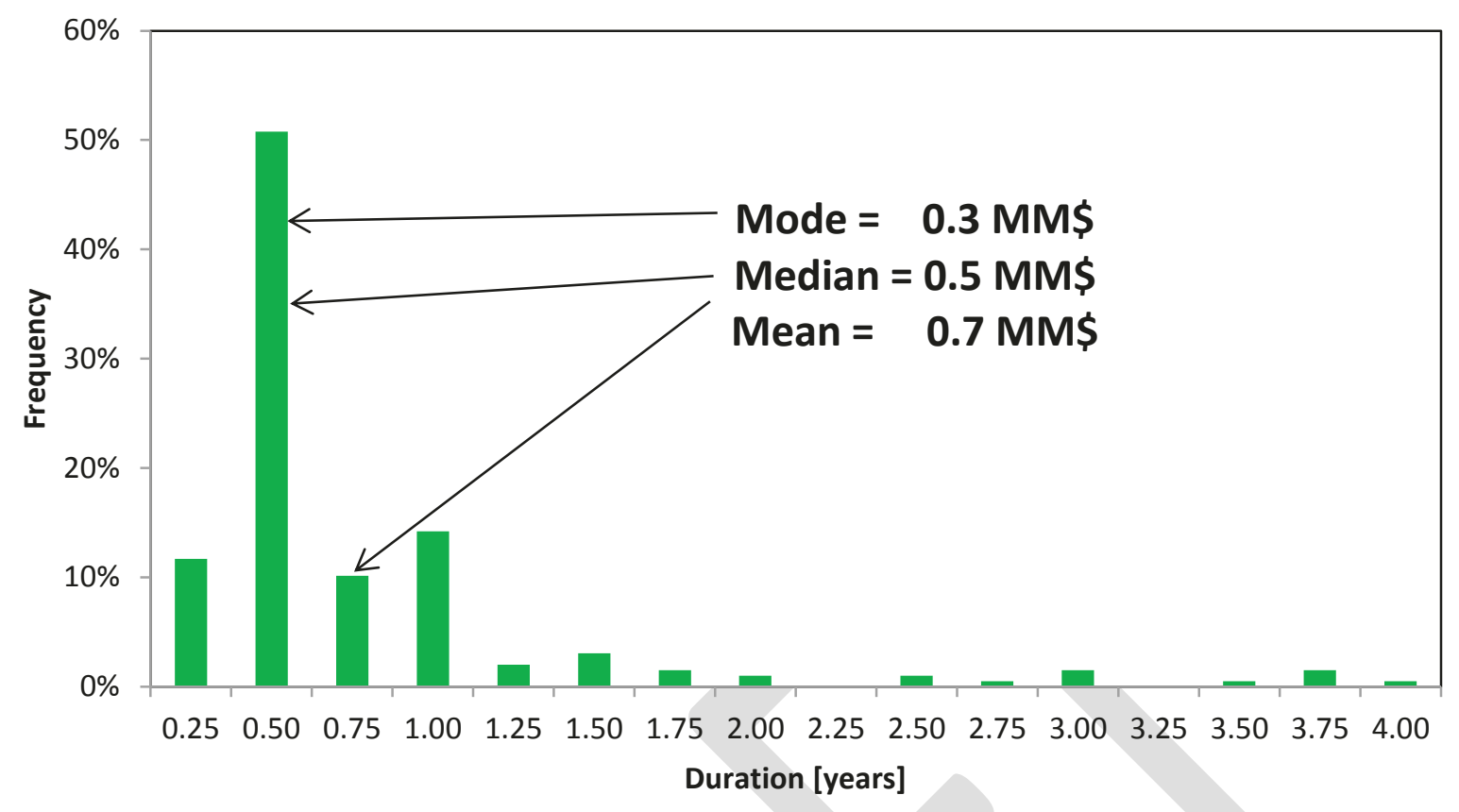

Figure 14: Cost/Year Distribution

\section{Integrated Research Project Proposed Work Scopes}

Five integrated research project work scopes were proposed:

1. Validation of Multi-physics/Multiscale Simulations - Experimental and analytical techniques for the characterization of tightly coupled phenomena to quantify and justify confidence in high fidelity simulations, the Idaho National Laboratory (3 years, 6.0MM\$)

2. Assessing Unit Resilience Impacts in the Optimal Control of Hybrid Systems, The Ohio State University (3 years, 4.5MM\$)

3. Modeling the Transport of Radionuclides in Arid Environments, AREVA-NP (3 years, 3.0MM\$)

4. Development of a Process For Tritium Separation and Capture from Aqueous Streams Containing Dilute Quantities of Tritium, AREVA-NP (3 years, 3.0MM\$)

5. Development of an Open-Source Virtual Reactor Road Map for Use in Research and Education, AREVA-NP (3 years, 3.0MM\$) 


\section{Qualitative Comments on the CINR Process}

Two proposers submitted qualitative statements concerning the administration of the Office of Nuclear Energy competitive funding programs. These statements are included here in their entirety with only editorial changes made for clarity.

\section{Fuel Cycle Research and Development}

There is significant work scope in many different areas, so, fewer topics that rotate so that awards can be funded at a higher level, e.g. 3 topics per year would be more beneficial. If the work scope is very specific to one or two issues, it results in more centered and relevant proposals.

\section{NEET}

Offer fewer topics with more dollars to maintain collaborations. The number of NEET subject areas has increased significantly from three to six. This reduces the number of proposals that can be funded in each area. If six or more areas are going to stay constant, the funding for NEET needs to be increase by a factor of two. If the funding is to stay the same for the same six subject areas, I would suggest that proposals be solicited for three subject areas the first year and the other three the second year.

Also, the original idea was to have each proposal include collaborators in industry, national laboratories, and universities. If that is expected, the funding needs to be increased to be similar to the amount of funding for IRPs at $1 \mathrm{MM} \$$ per year for three years. With funding so low, it is very difficult to have more than three collaborators without significantly reducing the scope that can be accomplished.

\section{Needed capabilities supporting research, training and technology \\ demonstration}

The capabilities needed to support research, training, and technology demonstration are state of the art characterization equipment that is maintained in working order.

- In addition, to be used for training, this equipment needs to be accessible for students.

- The capabilities must be able to handle low-level radioactive samples.

Capabilities that are needed include:

- Focused lon Beam Microscopes (FIB)

- Transmission Electron Microscopes, both high-resolution and analytical (TEM)

- Scanning Electron Microscopes (SEM)

- Electron Backscatter Diffraction (EBSD)

- Optical microscopy

- Mechanical Testing equipment

- Other specialized equipment such as Atom Probe Tomography (APT) 
Because these capabilities are in high demand for performing research, these are needed at national laboratories as well as universities.

\section{Additional Comments on DOE NEUP}

The funding of nuclear research by DOE NEUP is greatly appreciated and it has caused a sea change in the outlook of nuclear engineering departments across the country. The comments below should be taken in the context of this deep appreciation, while wanting to make the program even better, of higher quality and more useful to DOE.

\section{Relevance Assessment}

The system for assessing the relevance of research proposals in the DOE system is deeply flawed. The relevance reviewers appear to have little knowledge of the research currently being done in the DOE NEUP, so they reject proposals that they believe are being addressed when in fact they are not and recommend funding proposals that are repeats of others. Further, the review relevancy process is very opaque, as the PIs receive only a cryptic message of this work is being done elsewhere. This makes the DOE lose credibility with researchers.

- It is recommended that technical people be involved in both the real review and the relevancy evaluation as it is not possible to evaluate relevancy when the reviewers have little technical knowledge of the field.

\section{Accountability of research projects}

It is necessary to require greater accountability of the results in the research projects funded, especially on the IRP projects. This is because these projects often do not deliver on their promises. Currently there is little incentive in the DOE proposal process for being accurate on what is promised in the research proposal. If one promises more than can be reasonably delivered, but adjusts the milestones to something that can be fulfilled, little other checking appears to occur. In speaking to other researchers, the feeling is that there is little use of the reports we send in, the TPOC being busy with their own work and having little incentive to follow up.

This process is made worse in the initial evaluation process by the fact that the reviews are semi-blind, and it is not possible to take an applicant's track record into account while making the evaluation of whether the objectives of a research project is likely to be completed. NSF asks for a report on prior support; this should at least be evaluated by DOE.

\section{- It is recommended that DOE NEUP consider}

(i) No longer using the semi blind process of evaluations (find other means of awarding research funding to young investigators which is very a laudable 
goal - e.g. reserve a part of the budget for investigators less than X years out). This would allow reviewers to take the PI's track record into account in evaluating the proposal.

(ii) Ask for an account of prior support to be submitted with the proposal;

(iii) Require that IRPs have an Advisory Board approved by the TPOC who monitors the research projects and reports independently to DOE; this would allow in course corrections,

(iv) Change the reward system so that TPOC's have an incentive to engage in and monitor the research. 
Appendix 1: Summary data table for all proposals 


\begin{tabular}{|c|c|c|c|c|c|c|c|c|c|c|c|c|c|c|c|c|c|c|c|c|c|}
\hline \multicolumn{22}{|l|}{ 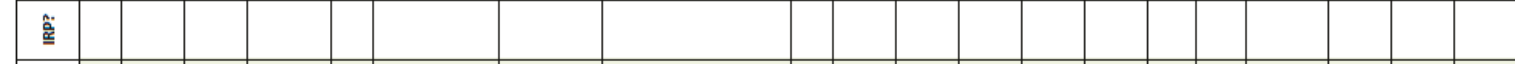 } \\
\hline 密 & & ه్ & $\stackrel{\bigsqcup}{0}$ & בิ & ه్రి & 骂 & $\nexists$ & ํㅗㅇ & & & & & 앙 & ํํㅇ & 영 & 吕 & ํํㅇ & 8 & 8 & 8 & 8 \\
\hline 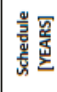 & w & N & $m$ & $m$ & 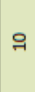 & in & $m$ & $m$ & & $\mid \begin{array}{l}n \\
m\end{array}$ & $\underset{\exists}{*}$ & $m$ & $\sim$ & $\sim$ & $\sim$ & $\sim$ & $\sim$ & $\sim$ & $\stackrel{\sim}{\text { I }}$ & $\stackrel{\sim}{\mu}$ & $\stackrel{i}{n}$ \\
\hline 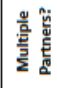 & 쓰 & $>$ & $>$ & $z$ & $>$ & $>$ & $>$ & $>$ & & $>$ & z & $>$ & $>$ & $>$ & $>$ & $>$ & $>$ & $>$ & $>$ & $>$ & $>$ \\
\hline 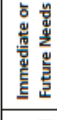 & - & 4 & - & 4 & 4 & 4 & 4 & 4 & & - & - & - & - & - & - & - & - & - & " & 4 & 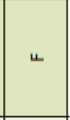 \\
\hline 落焉 & 岂 & N & $N$ & $\stackrel{\infty}{\circ}$ & $\infty$ & సं & $\underset{j}{n}$ & $\leadsto$ & & :ึ & $\begin{array}{l}\vec{m} \\
\vec{j} \\
\vec{H}\end{array}$ & \& & $\stackrel{\infty}{\circ}$ & $\rightarrow$ & $\rightarrow$ & -7 & 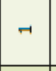 & N & नै & $\stackrel{m}{\mu}$ & $\stackrel{\perp}{ }$ \\
\hline 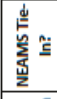 & $z$ & $z$ & $>$ & $>$ & $z$ & $>$ & $>$ & z & & $>$ & $z$ & $z$ & $z$ & z & $z$ & $z$ & $z$ & z & z & z & z \\
\hline 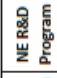 & & & & & & & & & & & & & & & & $\ddot{\check{c}}$ & $\ddot{\Psi}$ & & 立司 & 悥是 & 䇋昆 \\
\hline 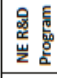 & $\ddot{x}$ & $\ddot{x}$ & $\ddot{x}$ & $\breve{u}$ & $u$ & 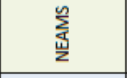 & $\ddot{\widetilde{u}}$ & $\frac{1}{\mathrm{u}}$ & & $\ddot{\ddot{x}}$ & 䇋导 & 离妾 & 竞 $\theta$ & 䇋 & $\frac{1}{\mathbf{L}} E$ & 迨 & 这 & u & $\ddot{\varkappa}$ & $\ddot{\widetilde{\alpha}}$ & $\ddot{\mathscr{x}}$ \\
\hline 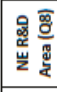 & & & ž & & $\frac{\vec{b}}{2}$ & 兽 & 盖 & 品 & & 尊 & & $\frac{\hbar}{2}$ & $\underline{\Sigma}$ & & & 善 & 盖 & $\frac{\vec{\mu}}{2}$ & 奠 & $\frac{.}{2}$ & 奠 \\
\hline 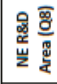 & $\bar{E}$ & 六 & 盖 & $\frac{\vec{m}}{2}$ & $\frac{\bar{m}}{2}$ & $\underline{\xi}$ & $\frac{\bar{t}}{2}$ & 5 & & $\frac{5}{2}$ & 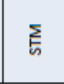 & 尊 & $\xi$ & $\Sigma_{n}^{\Sigma}$ & 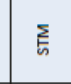 & $\sum_{5}^{5}$ & 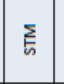 & 岁 & $\frac{\vec{\mu}}{z}$ & $\frac{1}{2}$ & ì \\
\hline 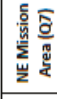 & & & & & $m$ & & $H$ & + & & $\sim$ & H & 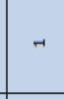 & N & $\rightarrow$ & H & $\sim$ & & & & & \\
\hline 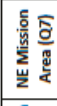 & - & $\sim$ & T & $m$ & N & $\sim$ & N & $m$ & & - & N & $\sim$ & - & $\sim$ & $\sim$ & -7 & $H$ & $m$ & N & $\sim$ & $\sim$ \\
\hline 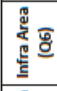 & & & & 蒿 & & $\frac{x}{\mathrm{~d}}$ & & & & & & & $\frac{.0 .}{2}$ & $\overrightarrow{\widetilde{d}}$ & & & & & & & \\
\hline 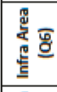 & & & ฮั & $\frac{.0 .}{2}$ & 㟧 & 递 & $\stackrel{u}{\underline{x}}$ & & & 崖 & $\vec{\varpi}$ & & 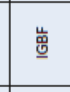 & $\underline{\underline{\underline{0}}}$ & ఫ్ర & & & व्य & & & \\
\hline 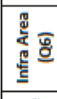 & 啇 & 좃 & 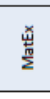 & 崌 & 조 & 좃 & 崖 & $\frac{z}{a}$ & & \begin{tabular}{|l|l}
$\underline{\underline{a}}$ \\
\end{tabular} & $\frac{0.0}{\frac{10}{2}}$ & 㟧 & 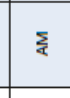 & $\frac{x}{\frac{x}{2}}$ & $\frac{\frac{x}{2}}{2}$ & $\overrightarrow{\widetilde{x}}$ & $\overrightarrow{\widetilde{\alpha}}$ & 弟 & 줏 & 崖 & $\stackrel{\mathscr{u}}{x}$ \\
\hline 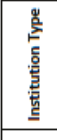 & 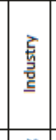 & $\begin{array}{l}\text { 总 } \\
\text { 裳 }\end{array}$ & $\begin{array}{l}\text { 意 } \\
\text { 常 }\end{array}$ & $\begin{array}{l}\text { 意 } \\
\text { 章 }\end{array}$ & 差意 & 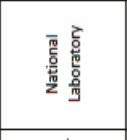 & $\begin{array}{l}\text { 意 } \\
\text { 旁 }\end{array}$ & 袁 & & 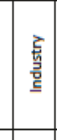 & 嗙 & 总 & 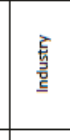 & 总 & 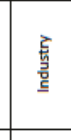 & 总 & 总毫 & 意 & 意 & 总 & 虔 \\
\hline 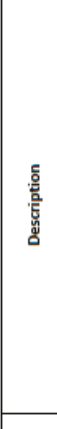 & 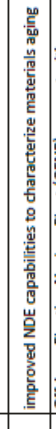 & 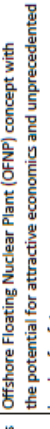 & 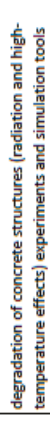 & 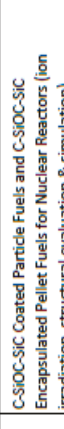 & & 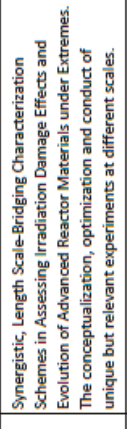 & 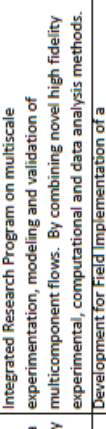 & 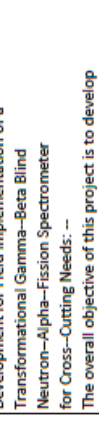 & 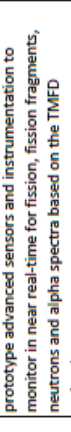 & 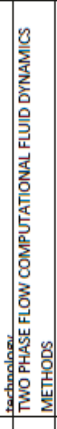 & 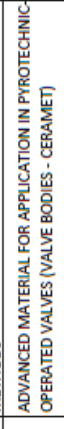 & 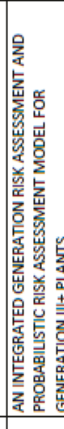 & 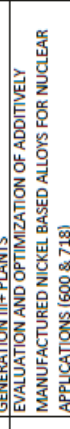 & 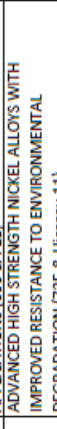 & 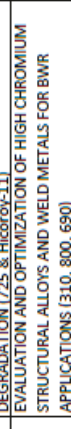 & 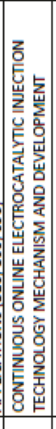 & 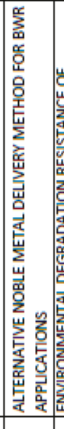 & 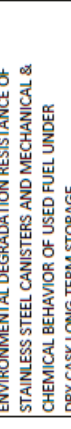 & 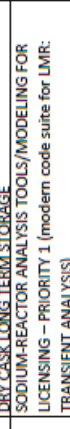 & 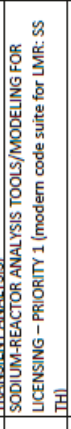 & 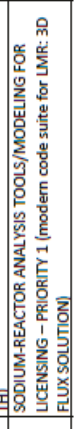 \\
\hline 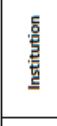 & $\overline{\overline{\tilde{w}}}$ & 递 & & 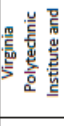 & $E$ & 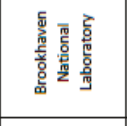 & 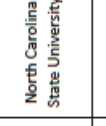 & 嫼 & & 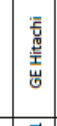 & 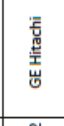 & 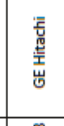 & 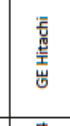 & 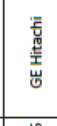 & 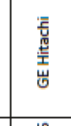 & 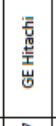 & 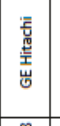 & 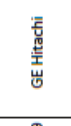 & 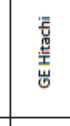 & 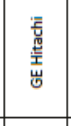 & 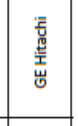 \\
\hline 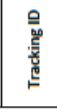 & 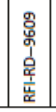 & 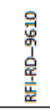 & 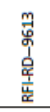 & 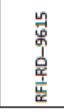 & 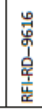 & 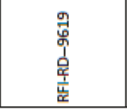 & 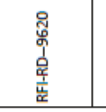 & 苟 & & 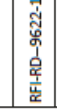 & 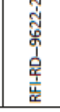 & 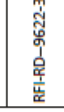 & 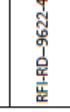 & 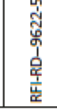 & 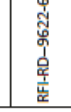 & 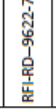 & 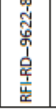 & 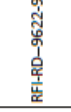 & 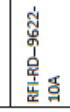 & 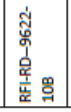 & 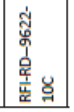 \\
\hline
\end{tabular}




\begin{tabular}{|c|c|c|c|c|c|c|c|c|c|c|c|c|c|c|c|c|c|c|c|c|c|c|c|}
\hline 产 & & & & & & & & & & & & & & & & & & & & & & & \\
\hline 喜 & $\stackrel{8}{8}$ & 8 & \&్తి & 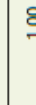 & & 8 & & & & & & & \begin{tabular}{|l|}
$g$ \\
ğ
\end{tabular} & సิ & సิ & సิ & $\hat{\tilde{\alpha}}$ & $\hat{\tilde{o}}$ & సิ & సิ & 콩 & సิ & ๑ே \\
\hline 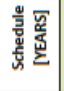 & $\stackrel{i}{i}$ & $\stackrel{\text { I }}{2}$ & $\stackrel{\sim}{\sim}$ & 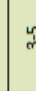 & & ๓ & $m$ & $m$ & $m$ & 㟧 & $m$ & 亗 & $m$ & $m$ & $m$ & $m$ & $m$ & m & $m$ & $m$ & $m$ & $m$ & $m$ \\
\hline 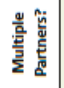 & $>$ & $>$ & $>$ & $>$ & & $>$ & $>$ & $>$ & $>$ & $>$ & $>$ & $\underline{\underline{z}}$ & $>$ & $>$ & $>$ & $z$ & $z$ & z & $z$ & z & $>$ & $>$ & $>$ \\
\hline 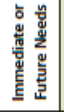 & 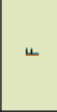 & 4 & 4 & - & & - & 4 & 4 & 4 & - & - & - & - & " & 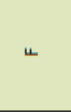 & 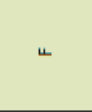 & 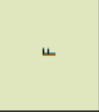 & 4 & 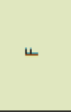 & u & - & - & - \\
\hline 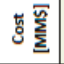 & $\stackrel{n}{I}$ & $\stackrel{n}{-1}$ & $i$ & $\frac{\infty}{j}$ & & $\stackrel{ \pm}{2}$ & जี & $\vec{b}$ & 品 & $\stackrel{\infty}{\circ}$ & $\stackrel{m}{i}$ & $\underline{\underline{z}}$ & $\stackrel{\oplus}{\top}$ & $\stackrel{\infty}{\circ}$ & $\stackrel{\infty}{\circ}$ & $\stackrel{\infty}{\circ}$ & $\stackrel{\infty}{0}$ & $\stackrel{\infty}{\circ}$ & $\stackrel{\infty}{\circ}$ & $\stackrel{\infty}{\circ}$ & $\stackrel{\infty}{\circ}$ & $\stackrel{\infty}{\circ}$ & $\sim$ \\
\hline 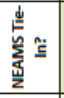 & z & z & $z$ & $z$ & & $z$ & $z$ & $z$ & $z$ & $z$ & $>$ & $z$ & $z$ & $z$ & $z$ & $>$ & $>$ & $>$ & $>$ & $>$ & $>$ & $>$ & z \\
\hline 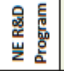 & 愛 & 䇋 & 度 & 妾 & 의 & 柁 & 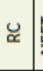 & 妾 $\mathrm{e}$ & 妾包 & & & & & $\breve{\varkappa}$ & $\breve{\varkappa}$ & 㞭它 & 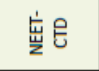 & & & $u$ & & & \\
\hline 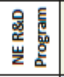 & $\ddot{\widetilde{x}}$ & $\ddot{\widetilde{x}}$ & $\ddot{\widetilde{\alpha}}$ & $\breve{a}$ & & $\ddot{\Psi}$ & $u$ & $\ddot{\varkappa}$ & $\breve{\propto}$ & 妾 & 妾 & $u$ & 庹主 & $u$ & $u$ & $\breve{\approx}$ & $\ddot{\ddot{\Sigma}}$ & $\breve{\varkappa}$ & $\ddot{\Psi}$ & 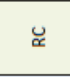 & $u$ & 営e & 㟧E \\
\hline 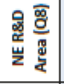 & 盖 & 关 & 盖 & दे & & छे & & & . & & & & $\xi$ & $\frac{\vec{y}}{2}$ & & & & 盖 & & 盖 & 盖 & & 5 \\
\hline 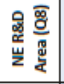 & 市 & 咨 & $\overrightarrow{\frac{\vec{z}}{z}}$ & 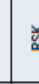 & 受 & $\underline{\underline{\underline{g}}}$ & $\overrightarrow{\frac{\vec{z}}{2}}$ & 立 & $\underline{\Sigma}$ & $\Sigma$ & ह & $\overrightarrow{\frac{1}{2}}$ & z & $\frac{\pi}{2}$ & $\overrightarrow{\frac{u}{z}}$ & 品 & $\frac{1}{2}$ & 产 & $\overrightarrow{\underline{z}}$ & $\frac{\vec{u}}{z}$ & $\overrightarrow{\vec{z}}$ & 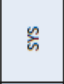 & $\underline{\underline{\underline{g}}}$ \\
\hline 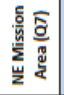 & & & & & & & & & & & N & & & $\sim$ & N & & & & & $m$ & & & $\sim$ \\
\hline 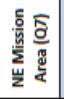 & N & $\sim$ & $\sim$ & . & & $\sim$ & $\sim$ & $\sim$ & N & $\sim$ & 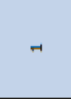 & $m$ & N & $m$ & $m$ & $m$ & $m$ & $\sim$ & $\sim$ & $\sim$ & $m$ & $m$ & - \\
\hline 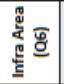 & & & & & & & & & & & & & z & & & $\frac{x}{2}$ & & & $\stackrel{0}{\underline{0}}$ & & & & \\
\hline 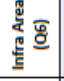 & & & & $\varepsilon^{z}$ & 8 & & & 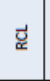 & & & 幽 & 岸 & 尊 & & & $\underline{\underline{\underline{\omega}}}$ & $\stackrel{\breve{u}}{\underline{x}}$ & $\stackrel{\breve{u}}{\underline{x}}$ & $\frac{z}{4}$ & & & & $\cong$ \\
\hline 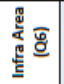 & 㟧 & 堂 & 稁 & $\frac{b}{2}$ & & $\frac{z}{4}$ & 惫 & 訔 & 兽 & $\frac{x}{\frac{x}{2}}$ & 噌 & 崌 & $\sum$ & $\stackrel{\breve{u}}{\underline{x}}$ & $\stackrel{\underline{\underline{x}}}{\underline{x}}$ & 줌 & 釬 & 岂 & 좃 & 좀 & 焙 & 崣 & 룽 \\
\hline 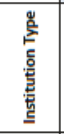 & 廍 & 总 & 旁 & 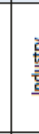 & & 䪰 & 怠 & 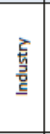 & 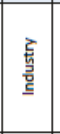 & 旁 & 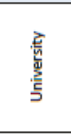 & 意 & 意 & 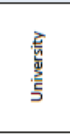 & 殿 & $\begin{array}{l}\text { 意 } \\
\text { 岁 }\end{array}$ & 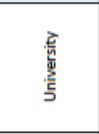 & 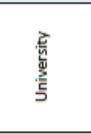 & $\begin{array}{l}\text { 意 } \\
\text { 常 }\end{array}$ & $\begin{array}{l}\text { 意 } \\
\text { 㟯 }\end{array}$ & 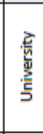 & 意 & 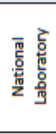 \\
\hline $\begin{array}{l} \\
\mathrm{s} \\
\mathrm{s} \\
\mathrm{g} \\
\mathrm{g} \\
\mathrm{g} \\
\end{array}$ & 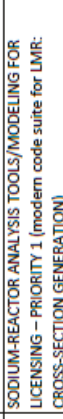 & 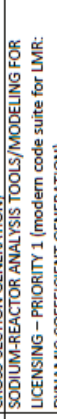 & & 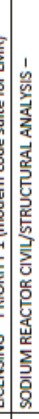 & & & 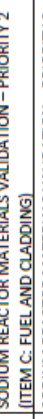 & 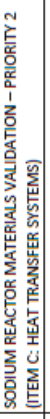 & 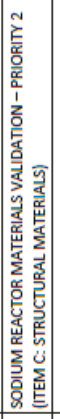 & 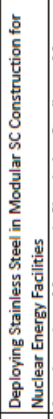 & 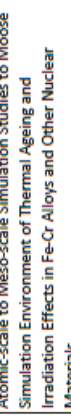 & 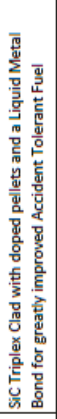 & 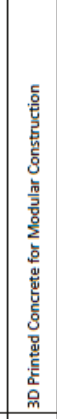 & 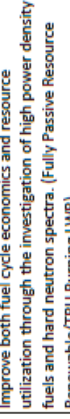 & 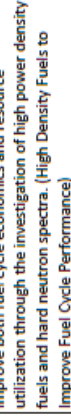 & 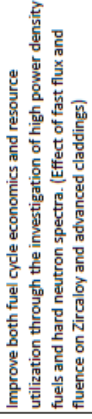 & 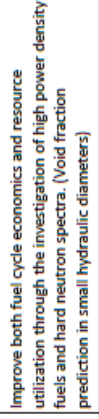 & 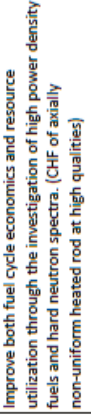 & 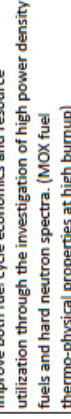 & 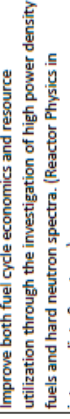 & 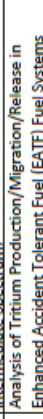 & 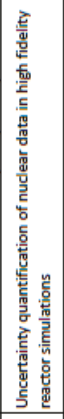 & 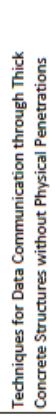 \\
\hline 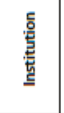 & 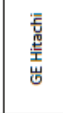 & 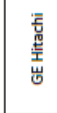 & 离 & 党 & 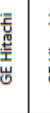 & 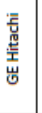 & 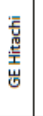 & 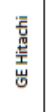 & 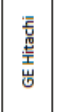 & 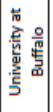 & 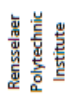 & 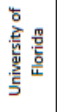 & 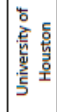 & 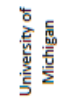 & 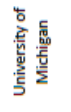 & 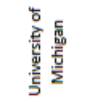 & 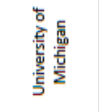 & 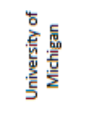 & 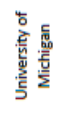 & 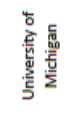 & . & & \\
\hline 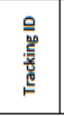 & 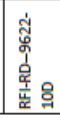 & 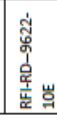 & & & & & & & 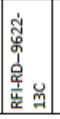 & 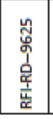 & 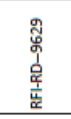 & 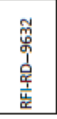 & 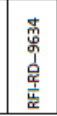 & 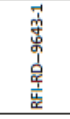 & 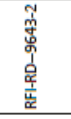 & 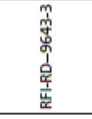 & 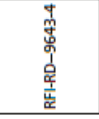 & 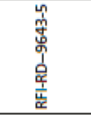 & 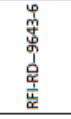 & 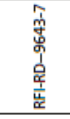 & 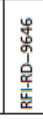 & 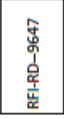 & 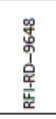 \\
\hline
\end{tabular}




\begin{tabular}{|c|c|c|c|c|c|c|c|c|c|c|c|c|c|c|c|c|c|c|c|c|c|c|}
\hline 产 & & & & & & & & & & & & & & & & 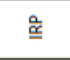 & & & & & & \\
\hline 䒽 & & & & 刕 & & $\tilde{\tilde{y}}$ & & & & & 영 & ํํㅇ & & 공 & 금 & 8 & f & ఫิ & 睘 & M․ & 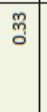 & ભ్ \\
\hline 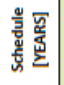 & $\underline{\underline{z}}$ & 崩 & 㟧 & $m$ & $\underline{\underline{z}}$ & in & 荘 & & & r & $\rightarrow$ & $m$ & 㟧 & $m$ & $\sigma$ & J & $m$ & $m$ & $m$ & $m$ & $m$ & $m$ \\
\hline 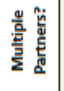 & $>$ & 岂 & 亗 & $>$ & $>$ & $>$ & $z$ & & & & $z$ & $>$ & z & $>$ & $z$ & $>$ & $>$ & $>$ & $z$ & $z$ & $z$ & $z$ \\
\hline 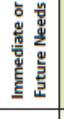 & - & - & - & - & - & - & - & & & & ${ }^{4}$ & - & - & - & - & - & - & - & ${ }^{4}$ & - & 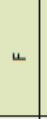 & 4 \\
\hline 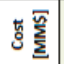 & 㟧 & 亗 & 㟧 & -1 & 崩 & $\stackrel{\infty}{0}$ & $\underline{\mathbf{u}}$ & & & 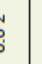 & - & $\eta$ & 岁 & $\stackrel{n}{n}$ & $\stackrel{\circ}{\circ}$ & in & $\stackrel{\sim}{\exists}$ & $\stackrel{\infty}{0}$ & 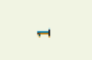 & $-H$ & - & - \\
\hline 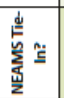 & $z$ & $z$ & $>$ & $z$ & $z$ & $z$ & $z$ & & & & $z$ & $z$ & $z$ & $z$ & $z$ & $>$ & z & $>$ & $z$ & $z$ & $z$ & $z$ \\
\hline 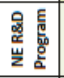 & & & & & & & & & & & & & & & 这e & $\ddot{\varkappa}$ & & $u$ & & & $u$ & \\
\hline 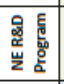 & 䇾 & 営 & $\ddot{u}$ & 接 & 授是 & 忘 & 岸 & & & 2 & $\ddot{\varkappa}$ & 产的 & $\ddot{\widetilde{x}}$ & $u$ & $u$ & $\begin{array}{l}\frac{n}{y_{1}^{2}} \\
\frac{1}{2}\end{array}$ & 岀 & $\begin{array}{l}\sum_{1}^{n} \\
\frac{1}{2}\end{array}$ & $u$ & 妾 & $\ddot{\propto}$ & $\ddot{\Psi}$ \\
\hline 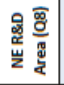 & 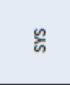 & 尊 & 党 & 㒸 & 总 & 前 & & & & & & & & & 5 & & 尊 & & 点 & 盖 & 益 & \% \\
\hline 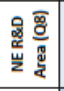 & $\underline{\underline{\underline{ }}}$ & $\frac{\vec{z}}{z}$ & $\frac{\vec{y}}{2}$ & $\stackrel{u}{\underline{~}}$ & $\underline{\underline{u}}$ & $\underline{\underline{\underline{u}}}$ & $\stackrel{\underline{u}}{\underline{m}}$ & & & 5 & 立 & 奠 & 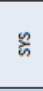 & $\frac{5}{3}$ & 岂 & E & $\underline{\underline{\underline{ }}}$ & $\frac{\overrightarrow{\mathrm{z}}}{\mathrm{z}}$ & 岂 & $\frac{\pi}{2}$ & i & in \\
\hline 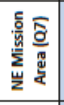 & a & & & $\sim$ & N & N & $\sim$ & & & & & & & & & N & a & & & m & & \\
\hline 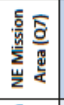 & H & $m$ & m & - & - & H & - & & & n & a & $\sigma$ & - & m & $m$ & - & H & m & $m$ & - & - & N \\
\hline 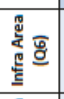 & & & & & & & & & & & & & & $\frac{\underline{x}}{\frac{u_{2}}{2}}$ & & & & & & & & \\
\hline 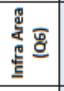 & $\frac{2}{4}$ & & & & $\frac{z}{4}$ & $\frac{z}{4}$ & & & & 3 & 隺 & & & ఫ్జ & & & $\frac{z}{4}$ & & & & & $\underline{\underline{u}}$ \\
\hline 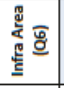 & $\underline{\underline{\underline{u}}}$ & & 焙 & $\stackrel{\underline{u}}{\underline{u}}$ & $\underline{\underline{\underline{g}}}$ & $\dddot{\underline{\underline{ }}}$ & 崖 & & & 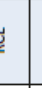 & 寅 & & & 容 & 崌 & 좊 & $\underline{\underline{\underline{u}}}$ & $\frac{x}{\frac{10}{2}}$ & 㟒 & 瑶 & $\cong$ & 㟯 \\
\hline 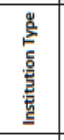 & 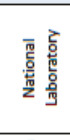 & 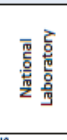 & 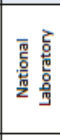 & 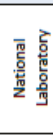 & 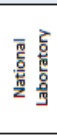 & 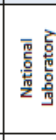 & 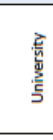 & & & & 喜 & 意 & $\begin{array}{l}\text { 意 } \\
\text { 常 }\end{array}$ & 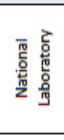 & 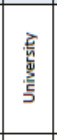 & 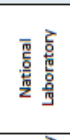 & 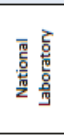 & 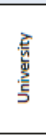 & $\begin{array}{l}\text { 蒡 } \\
\underline{\underline{\underline{z}}}\end{array}$ & 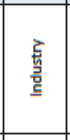 & 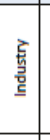 & 醇 \\
\hline 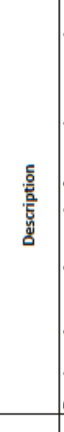 & 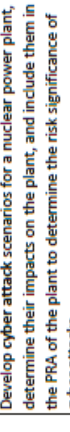 & 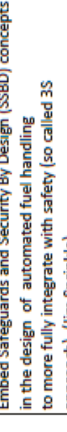 & 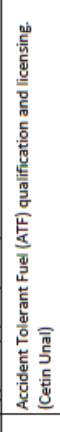 & 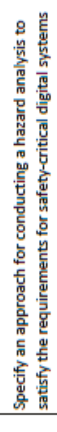 & 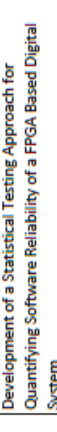 & 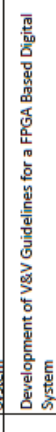 & 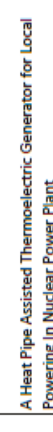 & & & . & 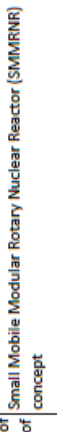 & 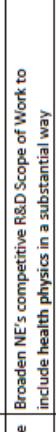 & 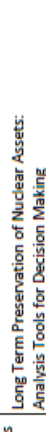 & 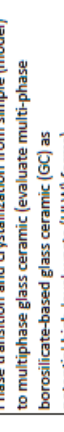 & 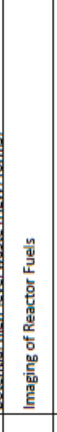 & 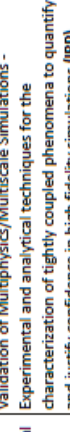 & 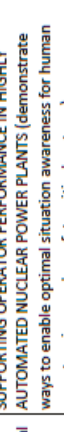 & 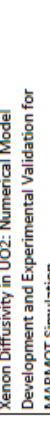 & 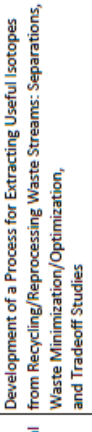 & 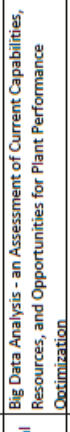 & 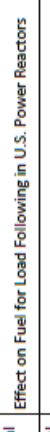 & 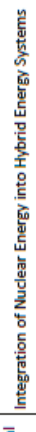 \\
\hline 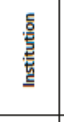 & 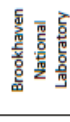 & & & & & & & & & & 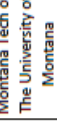 & 䧺总 & & 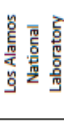 & 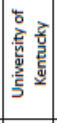 & 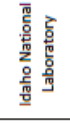 & 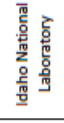 & 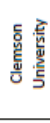 & 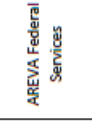 & 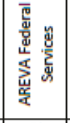 & 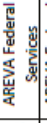 & \\
\hline 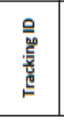 & 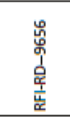 & 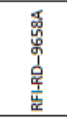 & 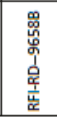 & 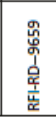 & 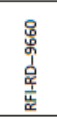 & 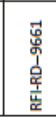 & 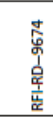 & & & & 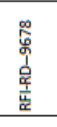 & 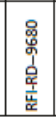 & 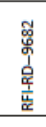 & 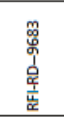 & 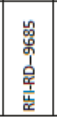 & 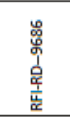 & 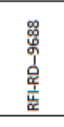 & 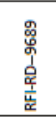 & 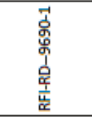 & 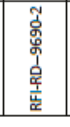 & 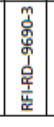 & \\
\hline
\end{tabular}




\begin{tabular}{|c|c|c|c|c|c|c|c|c|c|c|c|c|c|c|c|c|c|c|c|c|c|c|c|c|}
\hline 鋅 & & & & & & & & & & & 을 & $\underline{\underline{\underline{\alpha}}}$ & $\underline{\underline{\underline{\alpha}}}$ & & & & & & & & & & & \\
\hline 屁 & ח̊ & ֻू & ֻू & 墒 & 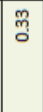 & 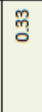 & 営 & \begin{tabular}{|l}
$m$ \\
$\tilde{O}$
\end{tabular} & \begin{tabular}{|l|l|}
\multirow{m}{m}{} & \\
\end{tabular} & 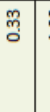 & 8 & ه్తి & ְ. & & & ¿ & :̊ํㅇ & I্ & & ڤ̊ & 8 & $\mathscr{\Xi}_{0}$ & 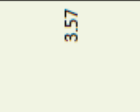 & సิ \\
\hline 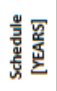 & $m$ & $m$ & $m$ & $m$ & $m$ & $m$ & m & $m$ & $m$ & $m$ & $m$ & $m$ & $m$ & $m$ & घे & $\sigma$ & $m$ & $m$ & ตे & 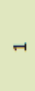 & N & + & r & $m$ \\
\hline 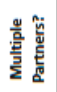 & $z$ & z & $z$ & z & $z$ & $z$ & z & $z$ & z & $z$ & $>$ & $>$ & $>$ & $>$ & $>$ & $>$ & $z$ & $>$ & $>$ & $>$ & $>$ & $>$ & $>$ & $>$ \\
\hline 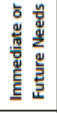 & - & " & - & - & - & - & 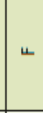 & ${ }^{u}$ & 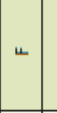 & 4 & 4 & - & 4 & - & - & 4 & 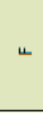 & - & - & 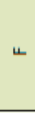 & ${ }^{u}$ & 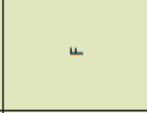 & u & 4 \\
\hline 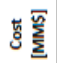 & -7 & 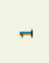 & $\rightarrow$ & -1 & -7 & -1 & -1 & -7 & $\rightarrow$ & $\rightarrow$ & $m$ & m & $m$ & 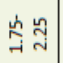 & $\overrightarrow{\dot{\omega}}$ & + & $\vec{i}$ & $\approx$ & 嵩 & ڤ̊ & N & $\stackrel{m}{m}$ & n & $\stackrel{\infty}{\circ}$ \\
\hline 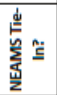 & $z$ & $z$ & $z$ & $z$ & $z$ & $z$ & $>$ & $z$ & z & $z$ & $z$ & $z$ & $>$ & z & $z$ & z & $z$ & z & $z$ & $z$ & $z$ & z & $z$ & $z$ \\
\hline 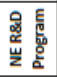 & & & & & & & & $\ddot{\Psi}$ & & & & & & & & & & & & & & & u & \\
\hline 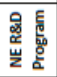 & $\ddot{\simeq}$ & $\breve{u}$ & 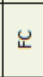 & 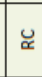 & $u$ & $u$ & $\ddot{\alpha}$ & $u$ & $u$ & $\ddot{\Psi}$ & $u$ & $u$ & $\ddot{\underline{x}}$ & 运园 & 立包 & $\breve{\approx}$ & $\breve{x}$ & $\underline{\mathscr{x}}$ & $\breve{\propto}$ & $u$ & $\breve{u}$ & $u$ & $\breve{\varkappa}$ & $u$ \\
\hline 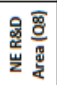 & 奠 & 歺 & & & 歺 & 善 & $\mid \overrightarrow{\frac{1}{2}}$ & 立 & 点 & $\frac{\pi}{2}$ & & $\underline{5}$ & $\frac{\vec{r}}{2}$ & 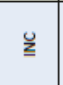 & $\stackrel{\underline{g}}{\underline{m}}$ & & & 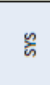 & 奠 & & 岂 & & $\frac{\vec{z}}{2}$ & 5 \\
\hline 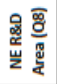 & $\underline{\underline{g}}$ & 岂 & 岂 & 气 & 岁 & $\frac{\vec{u}}{2}$ & 竞 & $\overrightarrow{\mathrm{u}}$ & $\mid \frac{\vec{J}}{2}$ & : & 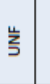 & 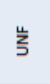 & ह & 5 & 㒸 & $\frac{i}{2}$ & $\frac{\pi}{2}$ & $\underline{\underline{u}}$ & $\stackrel{\underline{g}}{\underline{a}}$ & 岂 & $\frac{\vec{j}}{2}$ & $\frac{\vec{z}}{2}$ & $\frac{\pi}{2}$ & 岂 \\
\hline 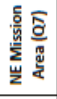 & N & & & & & & $m$ & $\sim$ & N & & & & $H$ & & & & & N & N & & & & & \\
\hline 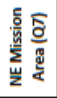 & - & $m$ & $m$ & -1 & $m$ & $m$ & $\sim$ & $m$ & m & $\sim$ & $m$ & $m$ & $\sim$ & $m$ & 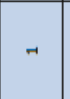 & $\sim$ & $\sim$ & Tr & - & $m$ & $m$ & $m$ & $\sim$ & $m$ \\
\hline 袁 & & & & & & & & & & & & & & & & & & & & & & & 容 & \\
\hline 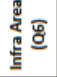 & & & & & & & & & 岂 & & & & & 㟧 & & & & $\frac{z}{q}$ & $\frac{z}{q}$ & & & 줖 & 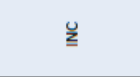 & \\
\hline 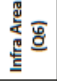 & $\dddot{\underline{\underline{m}}}$ & 岩 & & & $\vec{d}$ & 㟧 & × & $\simeq$ & \begin{tabular}{|l|} 
\\
\end{tabular} & 嵀 & $\vec{d}$ & 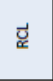 & х & $\frac{z}{\alpha}$ & $\underline{\underline{\underline{u}}}$ & 崖 & & $\stackrel{\underline{u}}{\underline{a}}$ & $\underline{\underline{u}}$ & 诺 & 崌 & 㟒 & 崖 & \\
\hline 旁 & 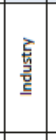 & 旁 & 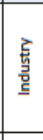 & 总 & \begin{tabular}{|l|} 
\\
$\underline{\underline{z}}$ \\
$\underline{\underline{\underline{\underline{\theta}}}}$
\end{tabular} & $\begin{array}{l}\text { 暜 } \\
\underline{\underline{\underline{\underline{n}}}}\end{array}$ & \begin{tabular}{|l}
$\underline{\underline{z}}$ \\
$\underline{\underline{z}}$ \\
$\underline{\underline{\underline{\theta}}}$
\end{tabular} & 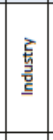 & \begin{tabular}{|c|}
$\underline{\underline{\underline{v}}}$ \\
$\underline{\underline{\underline{z}}}$ \\
\end{tabular} & $\begin{array}{l}\vec{z} \\
\underline{\underline{3}} \\
\underline{\underline{\underline{a}}}\end{array}$ & 总 & 暜 & 毫 & 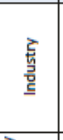 & 暜 & $\begin{array}{l}\text { 蒡 } \\
\underline{\underline{\underline{n}}}\end{array}$ & $\begin{array}{l}\underline{\underline{\underline{z}}} \\
\underline{\underline{\underline{\underline{\underline{\theta}}}}}\end{array}$ & 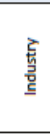 & $\begin{array}{l}\text { 暜 } \\
\underline{\underline{\underline{\theta}}}\end{array}$ & 童 & 䧛 & $\begin{array}{l}\text { 蒡 } \\
\underline{\underline{\underline{n}}}\end{array}$ & $\begin{array}{l}\text { 暜 } \\
\underline{\underline{\underline{\theta}}}\end{array}$ & 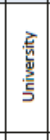 \\
\hline 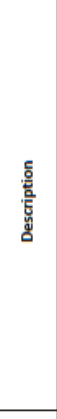 & 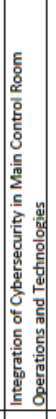 & 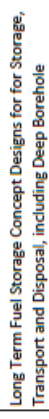 & & & & & 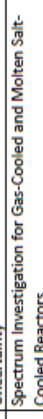 & 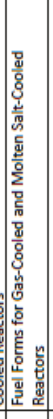 & 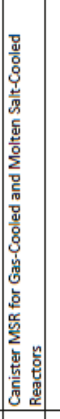 & 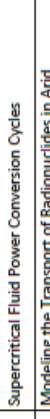 & & & 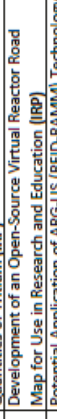 & & 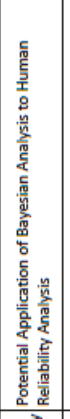 & 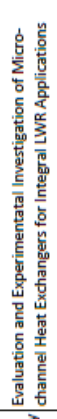 & 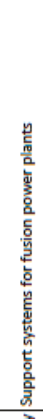 & 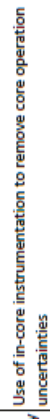 & 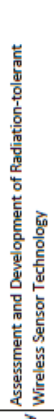 & & 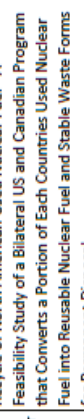 & 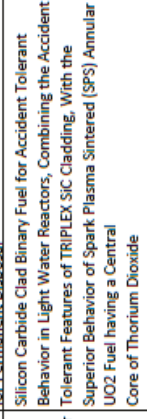 & 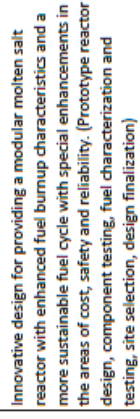 & 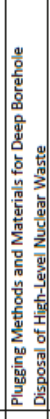 \\
\hline $\begin{array}{l}\frac{9}{2} \\
\underline{\underline{n}}\end{array}$ & | & 焉 & & & & & & & & & & & & & 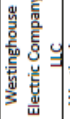 & & & & & & 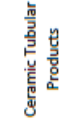 & 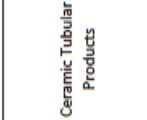 & 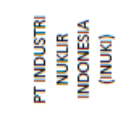 & 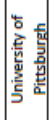 \\
\hline 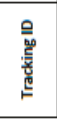 & 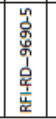 & 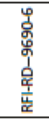 & \begin{tabular}{|l}
$\mathbf{S}$ \\
兽
\end{tabular} & 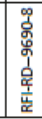 & 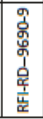 & & & & & & & 思 & & & 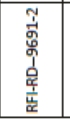 & 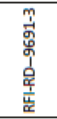 & 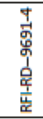 & 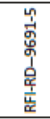 & 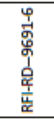 & 象 & 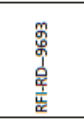 & 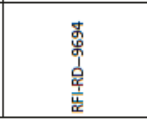 & 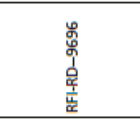 & 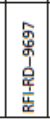 \\
\hline
\end{tabular}




\begin{tabular}{|c|c|c|c|c|c|c|c|c|c|c|c|c|c|c|c|c|c|c|c|c|c|c|}
\hline 弟 & & & & & & & & & & & & & & & & & & & & & & \\
\hline 离 & 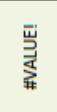 & ֻू & & & & & & & & & & & & ?: & 吕 & 8 & 응 & 号 & 8 & 8 & ळ్ & ํํㅇ \\
\hline 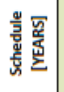 & 崩 & $m$ & 㟧 & 㟧 & 宸 & 㟧 & 宸 & w & 崩 & 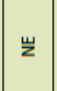 & 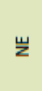 & 崩 & 㟧 & 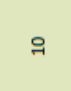 & $m$ & m & m & m & $m$ & $m$ & $m$ & m \\
\hline 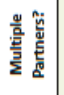 & $>$ & $>$ & $>$ & $>$ & $=$ & $>$ & $>$ & $>$ & $>$ & $>$ & $>$ & $>$ & $>$ & $>$ & $>$ & $>$ & $>$ & $>$ & $>$ & $>$ & $>$ & $>$ \\
\hline 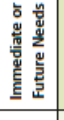 & 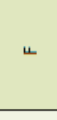 & ${ }^{4}$ & 4 & 4 & 4 & 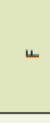 & 4 & 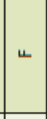 & - & - & 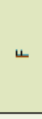 & 4 & แ & - & 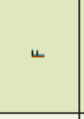 & - & - & 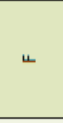 & $\stackrel{ }{4}$ & - & - & 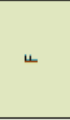 \\
\hline 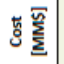 & 荘 & $r$ & $\underline{z}$ & $\underline{w}$ & w & 岂 & 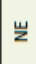 & $\underline{\underline{z}}$ & 㟧 & 荘 & 崩 & 岁 & 荘 & r & $\eta$ & $m$ & ก & ๆ & $m$ & $m$ & స & I \\
\hline 齿 & $z$ & $z$ & $z$ & $z$ & z & $>$ & $>$ & $>$ & $z$ & $z$ & $z$ & $z$ & $z$ & $z$ & $z$ & $z$ & $>$ & $z$ & $z$ & $>$ & $>$ & $z$ \\
\hline 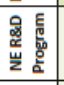 & & & & & & & & & & & & & & & & & & & & & & \\
\hline 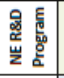 & $\ddot{u}$ & $\ddot{\varkappa}$ & $\breve{u}$ & $\mathscr{\varkappa}$ & $\ddot{\varkappa}$ & 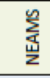 & $\begin{array}{l}\sum_{\underline{3}}^{n} \\
\end{array}$ & 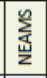 & 愛 & 岸 & 咅 & 这 & 妾 & $\breve{u}$ & $\breve{u}$ & $\ddot{\varkappa}$ & $\breve{\varpi}$ & $\ddot{\varkappa}$ & $\breve{\varpi}$ & $\begin{array}{l}\sum_{\underline{m}}^{n} \\
\underline{\underline{z}}\end{array}$ & $\begin{array}{l}\sum_{\mathbf{m}}^{n} \\
\end{array}$ & 立 \\
\hline 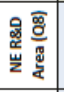 & 点 & 윰 & 首 & $\frac{\hbar}{2}$ & $\%$ & 总 & 㔛 & & & & & z & & & 5 & & 奠 & & & & & \\
\hline 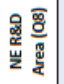 & $\frac{\vec{y}}{2}$ & 立 & $\frac{\bar{y}}{2}$ & \% & $\frac{\pi}{2}$ & ì & 立 & 益 & $\dddot{\underline{\underline{ }}}$ & $n$ & $\frac{\xi}{4}$ & 总 & $\frac{5}{2}$ & $\frac{\vec{u}}{2}$ & 岂 & ह & z & $\dddot{\breve{g}}$ & $\underline{\breve{~}}$ & 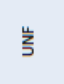 & $\frac{\vec{r}}{2}$ & 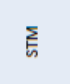 \\
\hline 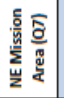 & & & & & & & & & N & $a$ & . & & & & & N & & & $\sim$ & & & \\
\hline 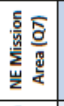 & $m$ & $\sim$ & $m$ & $\sim$ & $\sim$ & $\sim$ & $\sim$ & $\sim$ & 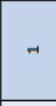 & 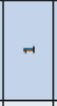 & $\sim$ & $\sim$ & $\sim$ & $m$ & $m$ & T. & T & ฯ & - & $m$ & $m$ & $\sim$ \\
\hline 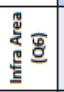 & & & & & & & & & & & & & & & & & & & & & & \\
\hline 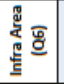 & & & & & & & & & $\stackrel{\varrho}{\underline{g}}$ & & & z & 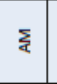 & & 己્జ & & ๖ & $\underline{\underline{\underline{u}}}$ & $\stackrel{\dddot{g}}{ }$ & & & 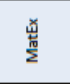 \\
\hline 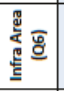 & 岂 & 崟 & 站 & 誓 & & 崖 & & & $\frac{z}{\alpha}$ & & $\sum$ & 畩 & $\frac{z}{4}$ & 釬 & 喜 & $\frac{.0}{\frac{10}{2}}$ & $\frac{.0}{\frac{10}{2}}$ & $\frac{z}{2}$ & $\frac{z}{\alpha}$ & 弟 & $\frac{x}{\frac{x}{2}}$ & $\Sigma$ \\
\hline 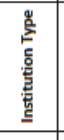 & 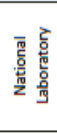 & 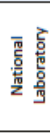 & 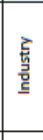 & 童 & 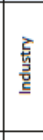 & 毫 & 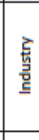 & $\begin{array}{l}\text { 烹 } \\
\underline{\underline{\underline{n}}}\end{array}$ & $\begin{array}{l}\text { 童 } \\
\underline{\underline{\underline{\underline{n}}}}\end{array}$ & $\begin{array}{l}\text { 总 } \\
\underline{\underline{\underline{z}}}\end{array}$ & 总童 & 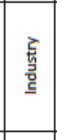 & 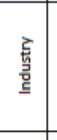 & 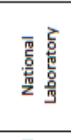 & 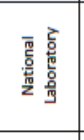 & 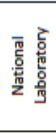 & 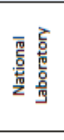 & 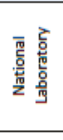 & 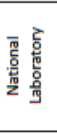 & 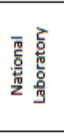 & 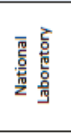 & 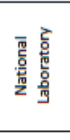 \\
\hline 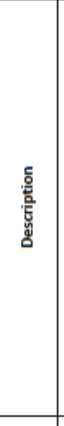 & 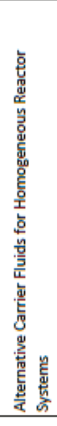 & 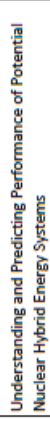 & 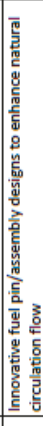 & & & 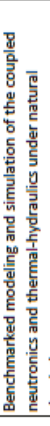 & 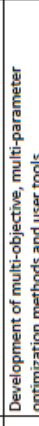 & 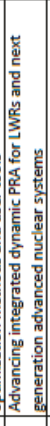 & 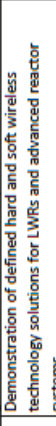 & 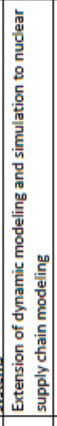 & 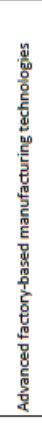 & 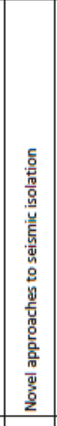 & 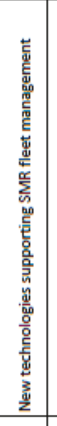 & 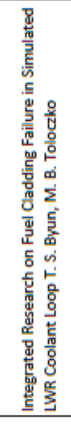 & 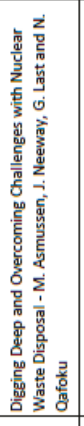 & 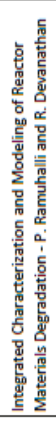 & 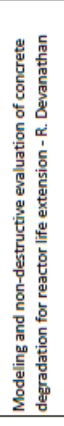 & 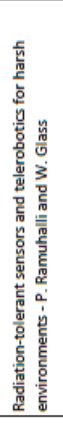 & 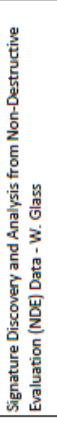 & 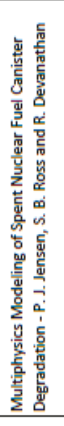 & 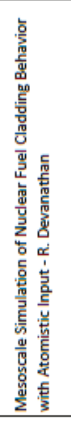 & 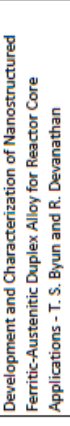 \\
\hline 厗 & & & & $\frac{3}{2}$ & $\frac{3}{2}$ & 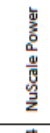 & 2 & 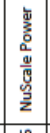 & 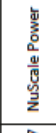 & 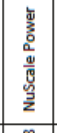 & 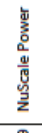 & 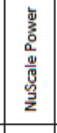 & 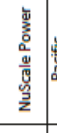 & & & & & & & & & \\
\hline 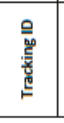 & 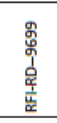 & 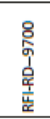 & 育 & 育 & ड़े & 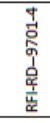 & 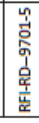 & 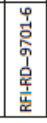 & 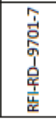 & 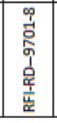 & 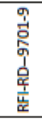 & 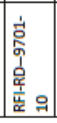 & 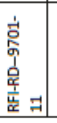 & 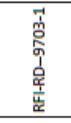 & 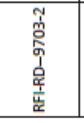 & 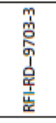 & 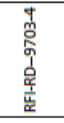 & 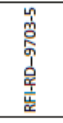 & 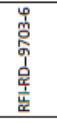 & 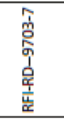 & 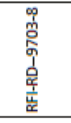 & 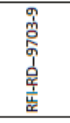 \\
\hline
\end{tabular}




\begin{tabular}{|c|c|c|c|c|c|c|c|c|c|c|c|c|c|c|c|c|c|c|c|c|c|}
\hline$\hat{\underline{\underline{\alpha}}}$ & & & & & & & & & & & & & & & & & & & & & \\
\hline 菐 & 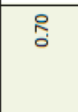 & 吕 & 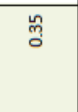 & Бे & 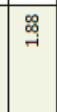 & 嗠 & 㟔 & 㟧 & 岵 & 岾 & 㟔 & $\begin{array}{l}9 \\
9\end{array}$ & 㔛 & ํㅗㅇ & 영 & ด̆ & 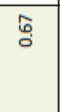 & 명 & 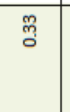 & 品 & ํํㅇ \\
\hline 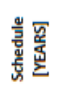 & $m$ & $m$ & $m$ & $m$ & $\sim$ & $m$ & $m$ & $m$ & $m$ & m & $m$ & $m$ & m & $\sim$ & $m$ & m & $m$ & N & $m$ & $m$ & $m$ \\
\hline 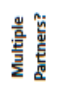 & $>$ & $>$ & z & $>$ & $>$ & z & $>$ & $>$ & $>$ & $>$ & $>$ & $>$ & $>$ & $>$ & > & $>$ & $>$ & $>$ & $>$ & $>$ & $>$ \\
\hline 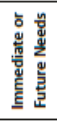 & 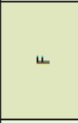 & - & 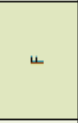 & 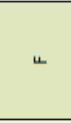 & u & 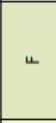 & - & - & - & แ & 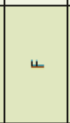 & - & - & - & - & - & 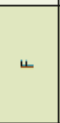 & - & 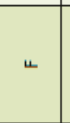 & L & 4 \\
\hline 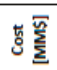 & $\overline{4}$ & 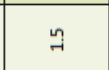 & 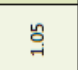 & $\sim$ & $\underset{n}{n}$ & $\begin{array}{l}n \\
\text { ñ } \\
0\end{array}$ & 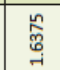 & 柋 & 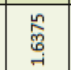 & 芯 & 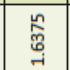 & $\approx$ & $\eta$ & - & 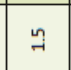 & $\eta$ & N & - & $\vec{r}$ & $\cong$ & 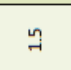 \\
\hline 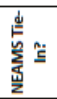 & z & $z$ & $z$ & z & z & z & z & z & z & z & $z$ & z & $z$ & z & z & z & z & z & $>$ & z & $z$ \\
\hline 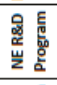 & & & & & 庶 & & u & $\ddot{\Psi}$ & $u$ & $\breve{\Psi}$ & u & & & & & & & $\breve{x}$ & 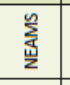 & & \\
\hline 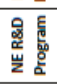 & 授 & 立 & 妾 & 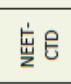 & $\ddot{\alpha}$ & u & 䇋 & 忘 & 悥 & 妾 $\mathrm{E}$ & 妾司 & 妾 & 立司 & 妾包 & 䇋 $E$ & 咅 E & $u$ & 妾 & $\breve{x}$ & $\breve{x}$ & $\ddot{\alpha}$ \\
\hline 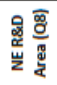 & 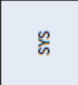 & 盖 & & & $\stackrel{\circ}{\frac{8}{2}}$ & 5 & $\frac{\vec{\mu}}{\underline{z}}$ & $\underline{\xi}$ & $\frac{\vec{u}}{\underline{z}}$ & $\stackrel{8}{\frac{8}{2}}$ & 5 & $\underline{E}$ & & 岁 & 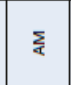 & & 差 & & 㕸 & & \\
\hline 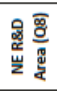 & 盖 & $\underline{\Sigma}$ & 岁 & ह & $\frac{\bar{z}}{2}$ & 岂 & $\frac{5}{4}$ & $\frac{5}{4}$ & $\frac{5}{4}$ & $\frac{5}{4}$ & s & $\frac{\vec{\mu}}{\underline{z}}$ & $\underline{\underline{u}}$ & $\%$ & $\underline{\underline{g}}$ & $\underline{\underline{g}}$ & 운 & 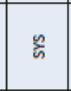 & $\frac{\vec{\mu}}{\underline{z}}$ & $\frac{\pi}{2}$ & $\frac{\vec{r}}{2}$ \\
\hline 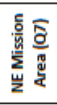 & $\sim$ & $\sim$ & $m$ & ה & & & & & & & & N & & & & & & $\rightarrow$ & & & \\
\hline 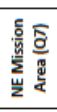 & - & m & - & - & $\sim$ & m & $m$ & N & $m$ & a & $m$ & $m$ & - & $m$ & + & + & $m$ & $\sim$ & N & N & $\sim$ \\
\hline 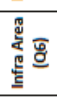 & & × & & & & & & & & & & & & & & & & & & & \\
\hline 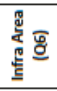 & & 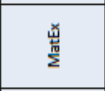 & 弟 & $\frac{z}{4}$ & & 岸 & 蒿 & 楌 & 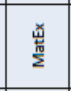 & 岂 & $\underset{\varpi}{\tilde{\alpha}}$ & $\frac{.0 .0}{\frac{10}{2}}$ & $\underline{\underline{ }}$ & 岂 & $\frac{z}{4}$ & $\frac{z}{4}$ & & & & & \\
\hline 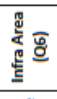 & & $\underline{\underline{\underline{\omega}}}$ & 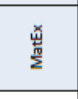 & 啇 & 崇 & $\vec{d}$ & s & 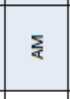 & $\frac{5}{4}$ & s & $\frac{5}{4}$ & $\underline{\underline{\underline{0}}}$ & $\frac{z}{4}$ & 弟 & $\underline{\underline{\underline{u}}}$ & $\underline{\underline{\underline{g}}}$ & $\vec{d}$ & & 岸 & 岸 & $\vec{d}$ \\
\hline 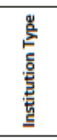 & 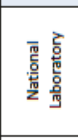 & 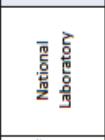 & 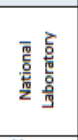 & 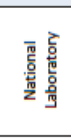 & 总 & 意 & 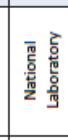 & 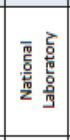 & 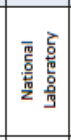 & 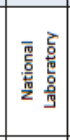 & 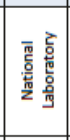 & 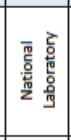 & 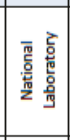 & 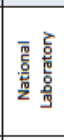 & 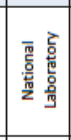 & 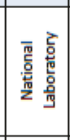 & 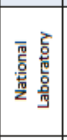 & 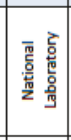 & 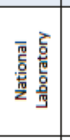 & 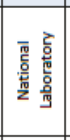 & 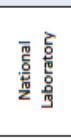 \\
\hline 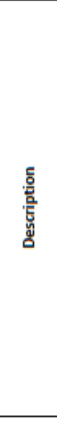 & 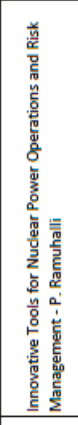 & 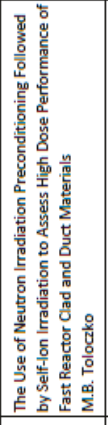 & 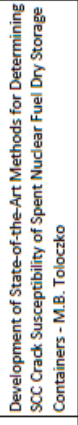 & 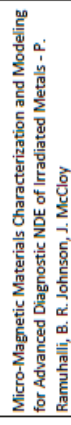 & 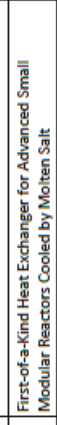 & 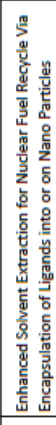 & 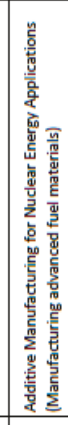 & 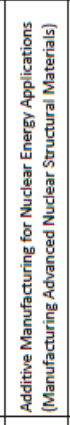 & 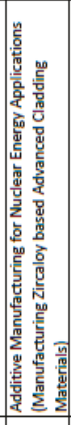 & 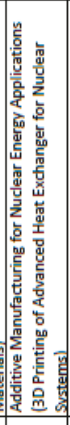 & 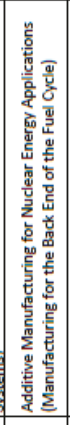 & 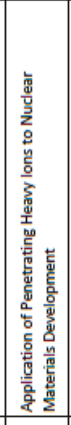 & 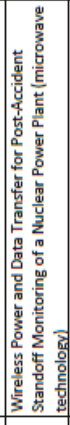 & 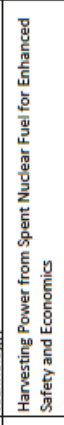 & 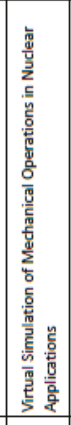 & 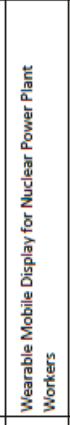 & 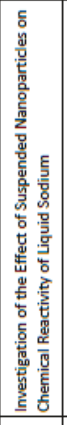 & 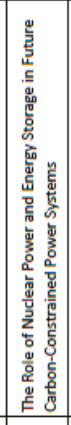 & 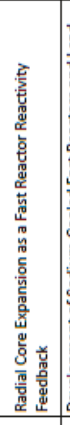 & 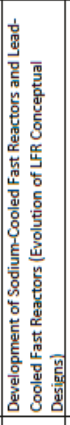 & 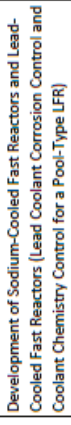 \\
\hline 旁 & & & & & & & & & & & & & & & & & & & & & 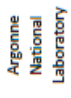 \\
\hline 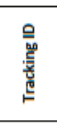 & 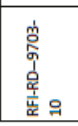 & 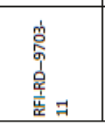 & 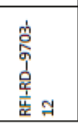 & 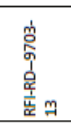 & 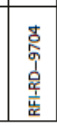 & 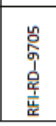 & 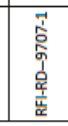 & 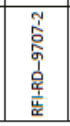 & 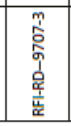 & 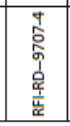 & 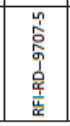 & 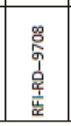 & 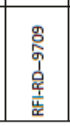 & 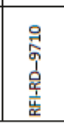 & 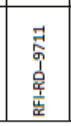 & 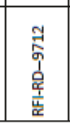 & 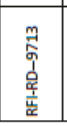 & 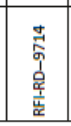 & 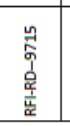 & 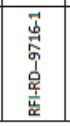 & 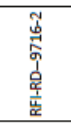 \\
\hline
\end{tabular}




\begin{tabular}{|c|c|c|c|c|c|c|c|c|c|c|c|c|c|c|c|c|c|c|c|c|c|c|c|c|}
\hline \multicolumn{25}{|l|}{$\hat{\underline{\underline{\underline{\underline{\mu}}}}}$} \\
\hline \multicolumn{25}{|l|}{ 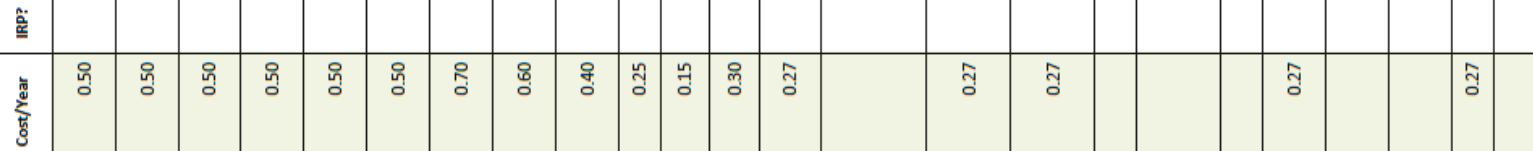 } \\
\hline 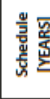 & m & $m$ & $m$ & $m$ & $m$ & $m$ & $\sigma$ & in & in & $m$ & $m$ & $m$ & $m$ & 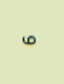 & $m$ & $m$ & $\sigma$ & $m$ & $m$ & $m$ & $\underline{z}$ & $m$ & $m$ & $m$ \\
\hline 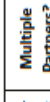 & $>$ & $>$ & $>$ & $>$ & $>$ & $>$ & $>$ & $>$ & $>$ & $>$ & $>$ & $>$ & $>$ & $>$ & $>$ & $>$ & $z$ & $>$ & $>$ & $>$ & $\underline{w}$ & $>$ & $z$ & $z$ \\
\hline 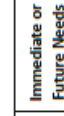 & 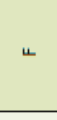 & 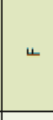 & 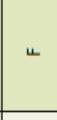 & $u$ & 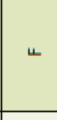 & $u$ & 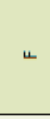 & $\stackrel{ }{L}$ & - & - & - & $L$ & - & - & - & - & - & - & - & - & - & - & 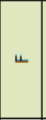 & 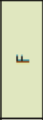 \\
\hline 落 & 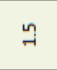 & $\eta$ & $\eta$ & $\eta$ & $\eta$ & $\eta$ & $\stackrel{\infty}{i}$ & $m$ & N & 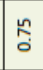 & 告 & 옹 & $\stackrel{\infty}{\circ}$ & 岁 & $\stackrel{\infty}{\circ}$ & $\stackrel{\infty}{\circ}$ & \begin{tabular}{|l}
$\infty$ \\
0 \\
0 \\
0 \\
0
\end{tabular} & $\begin{array}{l}\text { o } \\
\vdots \\
0 \\
0\end{array}$ & \begin{tabular}{|l|l} 
\\
0 \\
$\vdots$ \\
$\vdots$ \\
0
\end{tabular} & $\stackrel{\infty}{\circ}$ & $\underline{\mathrm{w}}$ & $\exists$ & $\stackrel{\infty}{0}$ & 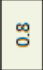 \\
\hline 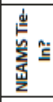 & $z$ & z & $z$ & z & $z$ & $z$ & $z$ & $z$ & z & $>$ & $z$ & $>$ & $z$ & $z$ & $>$ & $>$ & $\Rightarrow$ & $>$ & $>$ & z & $>$ & $z$ & $z$ & $z$ \\
\hline 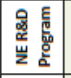 & & & & & & & & & & & & & & & u & $u$ & 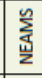 & & & & & $\ddot{\Psi}$ & & \\
\hline 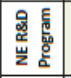 & $\mathscr{\Psi}$ & $\breve{\varkappa}$ & $\breve{\approx}$ & $\breve{\Psi}$ & $\breve{\approx}$ & $\breve{\approx}$ & $\breve{\approx}$ & 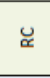 & u & $\ddot{\varkappa}$ & 阌 & 峞它 & $\breve{u}$ & $u$ & $\begin{array}{l}\sum_{\frac{1}{2}}^{n} \\
\end{array}$ & 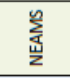 & u & $\begin{array}{l}\sum_{x}^{n} \\
\text { 妾 }\end{array}$ & \begin{tabular}{|l|} 
\\
\\
$\frac{n}{2}$
\end{tabular} & 岸E & 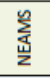 & 这 & $u$ & $\ddot{\Psi}$ \\
\hline 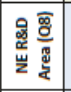 & & & 5 & & & & 美 & 奠 & & & & & $\sum$ & & & & & & $\frac{\vec{r}}{2}$ & 剪 & & 差 & 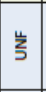 & $\cong$ \\
\hline 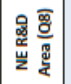 & ๕ & 운 & 嵌 & $\frac{\bar{r}}{2}$ & 咨 & $\frac{\vec{w}}{z}$ & 咨 & $\frac{i}{2}$ & $\overrightarrow{\frac{\vec{u}}{z}}$ & z & 党 & 盖 & $\overrightarrow{\frac{\vec{u}}{z}}$ & $\overrightarrow{\frac{\vec{z}}{2}}$ & $\frac{\vec{u}}{z}$ & $\frac{\vec{y}}{2}$ & $\%$ & $\frac{\vec{r}}{2}$ & 部 & $\overrightarrow{\vec{z}}$ & 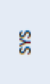 & $\underline{\underline{\underline{u}}}$ & $\frac{\vec{u}}{\mathbf{z}}$ & $\frac{5}{4}$ \\
\hline 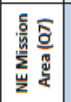 & & & & & & & & & & & & & & & + & $\sigma$ & & & & & & $\sim$ & & a \\
\hline 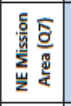 & N & $\sim$ & $\sim$ & $\sim$ & $\sim$ & $\sim$ & $\sim$ & $\sim$ & $m$ & 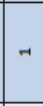 & 7 & 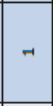 & $m$ & $m$ & $m$ & $m$ & $\rightarrow$ & $m$ & + & $m$ & + & 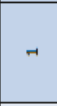 & $m$ & - \\
\hline 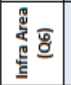 & & & & & & & $\underline{\underline{u}}$ & $\underline{\underline{\underline{u}}}$ & & & & & 嬛 & $\frac{.0}{2}$ & & & & & 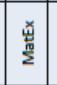 & & & & & $\dddot{\underline{g}}$ \\
\hline 离 & & & & & & $\frac{.0 .}{\frac{1}{2}}$ & $\frac{z}{4}$ & $\frac{z}{q}$ & $\underline{\underline{\underline{\omega}}}$ & z & & & 交 & $\vec{d}$ & $\frac{.0 .0}{2}$ & $\frac{.0 .0}{\frac{10}{2}}$ & 崇 & $\frac{.0 .0}{2}$ & × & & & $\frac{z}{4}$ & & $\sum$ \\
\hline 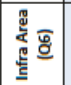 & 崖 & 崖 & $\overrightarrow{\widetilde{w}}$ & 岂 & 岂 & x & 岂 & 岸 & 曽 & 䒼 & & & 崌 & × & 岂 & 岂 & $\vec{\sigma}$ & 崌 & 중 & 䍘 & & $\underline{\underline{\underline{u}}}$ & 㟒 & $\frac{z}{\alpha}$ \\
\hline 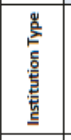 & 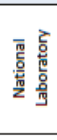 & 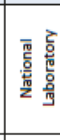 & 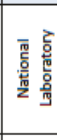 & 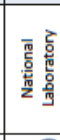 & 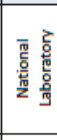 & 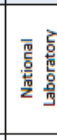 & 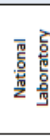 & 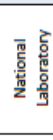 & 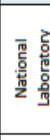 & 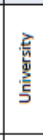 & 意 & 意 & 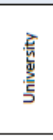 & 总 & 意 & 意 & 虽 & 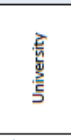 & 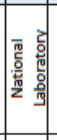 & 屡 & 意 & 喜 & 鹿 & 展 \\
\hline 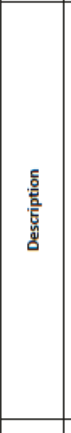 & 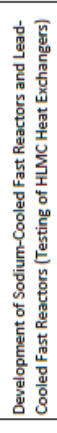 & 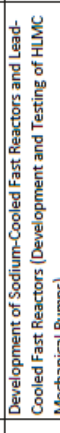 & 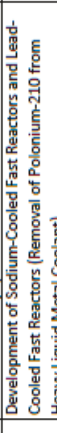 & 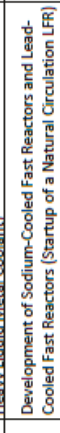 & 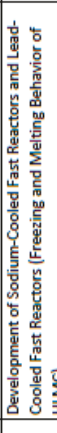 & 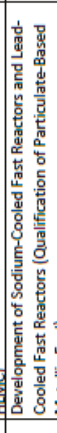 & 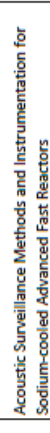 & 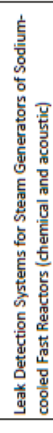 & 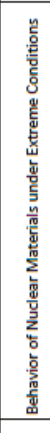 & 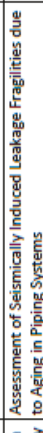 & 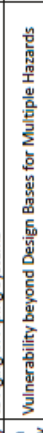 & 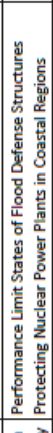 & 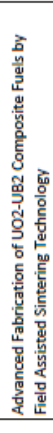 & 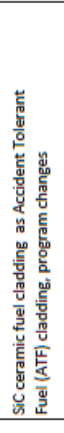 & 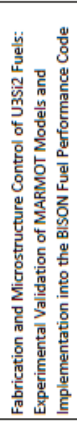 & 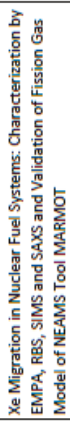 & 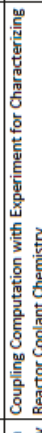 & 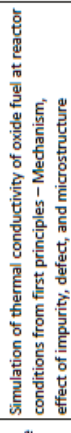 & 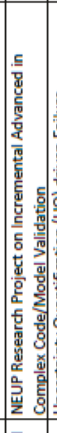 & 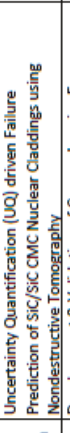 & & 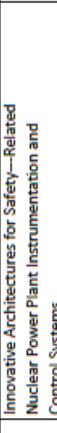 & 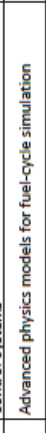 & 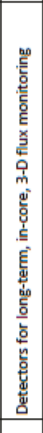 \\
\hline 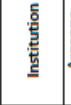 & & & & & & & & & & & & & & & 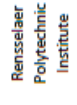 & 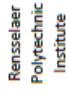 & & 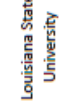 & & 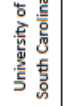 & 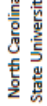 & & & 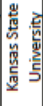 \\
\hline 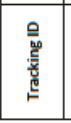 & 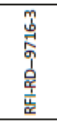 & 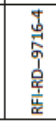 & 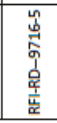 & 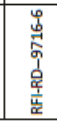 & 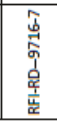 & 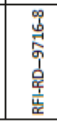 & $\begin{array}{l}\text { 疍 } \\
\text { 旁 }\end{array}$ & 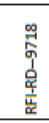 & 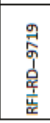 & 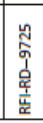 & 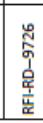 & 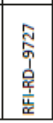 & 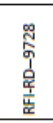 & 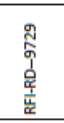 & 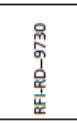 & 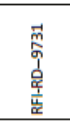 & 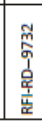 & 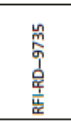 & 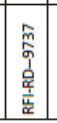 & 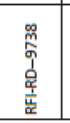 & 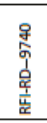 & 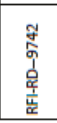 & 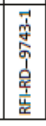 & 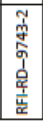 \\
\hline
\end{tabular}




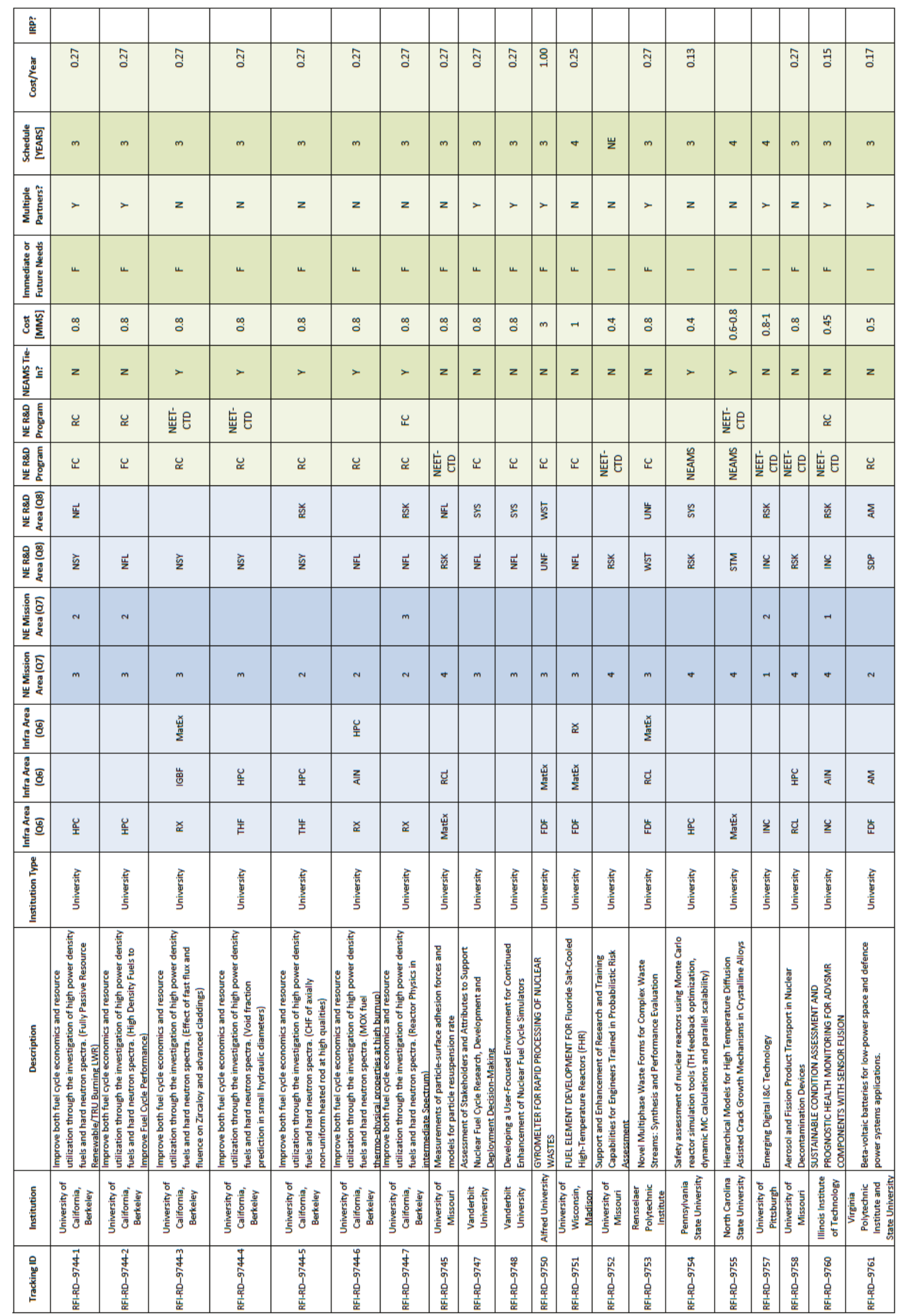




\begin{tabular}{|c|c|c|c|c|c|c|c|c|c|c|c|c|c|c|c|c|c|c|c|c|c|}
\hline 旁 & & & & & & & & & & & & & & & & & & & & & \\
\hline 產 & & 경 & Aิ & $\begin{array}{l}8 \\
+9\end{array}$ & $\underset{-1}{8}$ & ํㅗㅇ & ৪. & 8 & శ్ & 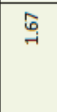 & : & f & ส & 8 & 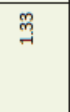 & 品 & & \& & 8 & & 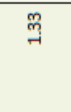 \\
\hline 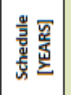 & $m$ & m & $m$ & $m$ & in & $m$ & $m$ & m & + & $m$ & + & $m$ & $\sim$ & & $m$ & $m$ & 崩 & $m$ & $m$ & 崩 & $m$ \\
\hline 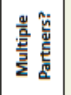 & $>$ & $>$ & $>$ & $>$ & $z$ & $>$ & $>$ & $>$ & $>$ & $>$ & $z$ & $z$ & $>$ & $>$ & $>$ & $>$ & $>$ & $>$ & $>$ & $>$ & $>$ \\
\hline 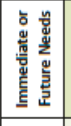 & - & - & - & - & - & - & - & - & - & - & - & ${ }^{4}$ & 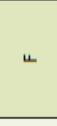 & 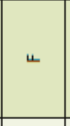 & 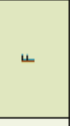 & - & - & - & - & 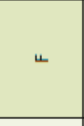 & - \\
\hline 裘产 & $\overrightarrow{\vec{\omega}}$ & in & $\stackrel{\infty}{\circ}$ & $\approx$ & in & $\eta$ & $\stackrel{\infty}{-}$ & $m$ & $\vec{n}$ & in & n & $\underset{\exists}{~}$ & ग & $m$ & + & I & 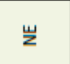 & $m$ & $m$ & $\underline{\underline{z}}$ & + \\
\hline 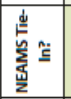 & $z$ & $z$ & $>$ & $z$ & $z$ & $z$ & $z$ & $z$ & $z$ & $>$ & $z$ & $z$ & $z$ & $z$ & $>$ & $>$ & $>$ & z & $z$ & $z$ & $z$ \\
\hline 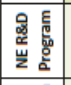 & & & & 立㦴 & $\ddot{\varkappa}$ & & & & & $\begin{array}{l}\text { 亗 } \\
\underline{\underline{w}}\end{array}$ & & & & & & & & & & & \\
\hline 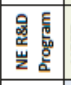 & 妾包 & $\breve{x}$ & 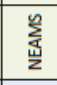 & u & 立e & 妾是 & 离导 & 度是 & $u$ & $u$ & u & 㐫e & $\breve{x}$ & 立妾 & $\begin{array}{l}\underline{n} \\
\frac{n}{2}\end{array}$ & $\begin{array}{l}\sum_{\mathrm{w}}^{n} \\
\mathrm{n}\end{array}$ & $\begin{array}{l}\frac{n}{2} \\
\frac{1}{2}\end{array}$ & u & u & u & 愛远 \\
\hline 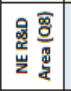 & 益 & $\frac{\pi}{2}$ & $\frac{\pi}{2}$ & 尊 & 曾 & & 율 & 兑 & 岂 & $\frac{\pi}{2}$ & 岂 & $\dddot{\underline{g}}$ & 욤 & 奠 & 尊 & 首 & 尊 & $\frac{n}{5}$ & g & 占 & 㒸 \\
\hline 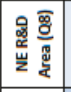 & $\cong$ & 奠 & 金 & $\frac{\vec{u}}{2}$ & $\frac{\bar{r}}{2}$ & $\underline{\underline{\underline{u}}}$ & $\underline{\underline{\underline{ }}}$ & $\stackrel{\underline{\underline{u}}}{ }$ & 5 & 萤 & 5 & 养 & $\frac{\bar{n}}{2}$ & z & 品 & 立 & $\frac{\bar{v}}{2}$ & 岁 & 岂 & 岂 & $\underline{\underline{\underline{u}}}$ \\
\hline 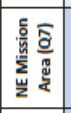 & a & & & + & & & & & & & & & & & & & & & & & $\sim$ \\
\hline 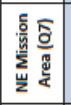 & - & $\Rightarrow$ & + & m & 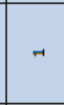 & a & N & $r$ & m & m & $m$ & + & N & + & + & + & + & $m$ & $m$ & m & + \\
\hline 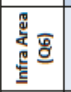 & & & & & & & & & & & & & & & & & & & & & \\
\hline 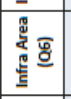 & & & & & & & $\frac{z}{4}$ & $\frac{z}{4}$ & 己ैజ & & ఫ్జ & $\frac{z}{4}$ & & 苔 & & & & & & 蔍 & \\
\hline 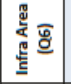 & $\dddot{\underline{g}}$ & 豆 & & & 垔 & $\frac{z}{4}$ & 喜 & $\underline{\underline{\underline{g}}}$ & 崌 & 釬 & 㟒 & $\underline{\underline{\underline{ }}}$ & & z & 喜 & & & 弟 & 弟 & క & $\underline{\underline{\underline{u}}}$ \\
\hline 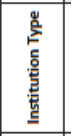 & 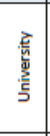 & 厝 & 展 & 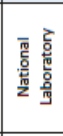 & 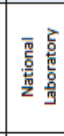 & 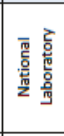 & 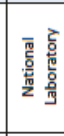 & 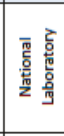 & 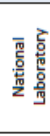 & 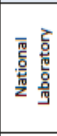 & 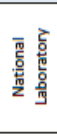 & 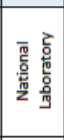 & 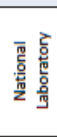 & 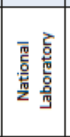 & 亳 & 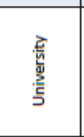 & 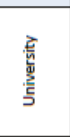 & 亳 & 居 & 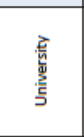 & 亳 \\
\hline 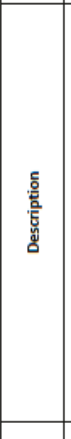 & 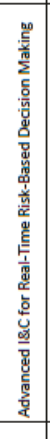 & & 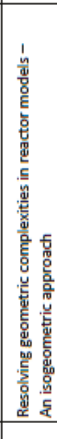 & 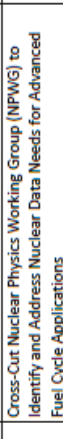 & 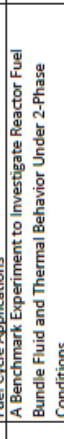 & 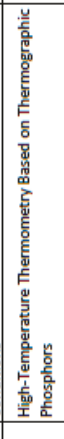 & 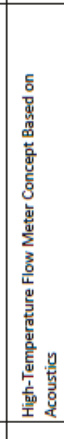 & 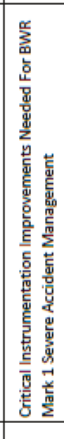 & 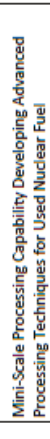 & 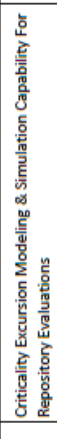 & 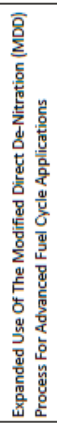 & 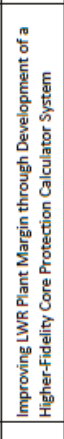 & 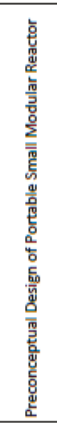 & 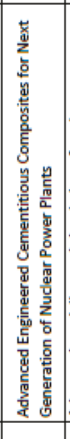 & 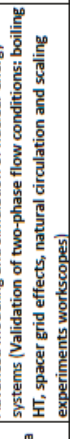 & 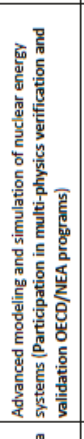 & 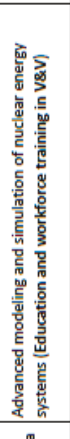 & 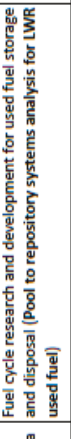 & 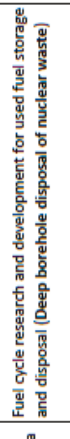 & 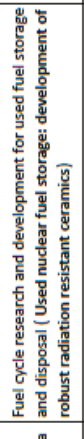 & 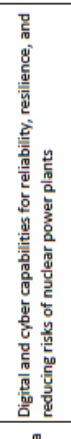 \\
\hline 喜 & & & 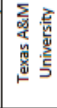 & & & & & & & & & & & & 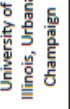 & 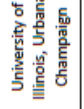 & 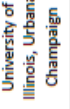 & 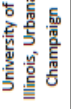 & 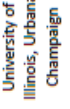 & 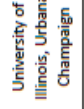 & 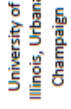 \\
\hline 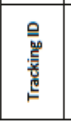 & 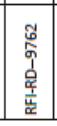 & 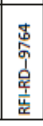 & 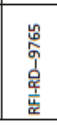 & 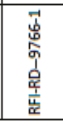 & 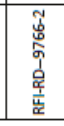 & 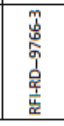 & 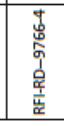 & 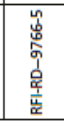 & 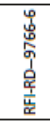 & 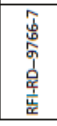 & 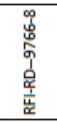 & 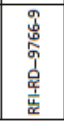 & $\begin{array}{l}\text { 总 } \\
\text { ô }\end{array}$ & 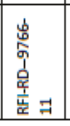 & 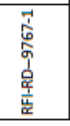 & 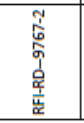 & 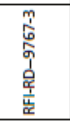 & 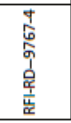 & 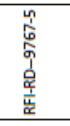 & 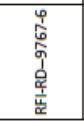 & 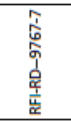 \\
\hline
\end{tabular}




\begin{tabular}{|c|c|c|c|c|c|c|c|c|c|c|c|c|c|c|c|c|c|c|c|c|}
\hline$\underline{\underline{\underline{\underline{a}}}}$ & & & & & & & & & & & & & & & & & & & & \\
\hline 悹 & & & & & 8 & 8 & \begin{tabular}{|l|} 
i \\
\end{tabular} & సิ & \$ & 范 & สి & 旁 & & శึ & 영 & 공 & 盟 & & & సิ \\
\hline 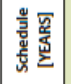 & $\underline{w}$ & $\underline{w}$ & 岁 & 崩 & $m$ & $m$ & in & $m$ & $m$ & $m$ & m & $m$ & $m$ & N & $m$ & $m$ & $m$ & $\frac{\mathrm{u}}{\mathrm{z}}$ & $m$ & $m$ \\
\hline 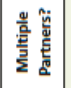 & z & z & z & $z$ & $>$ & $>$ & $>$ & $>$ & $>$ & $>$ & $>$ & z & $>$ & $z$ & $>$ & $>$ & $>$ & z & $>$ & $>$ \\
\hline 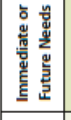 & 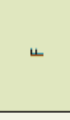 & แ & u & 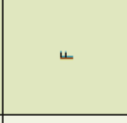 & - & - & - & u & - & ч & - & - & - & - & 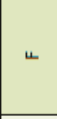 & 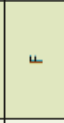 & แ & 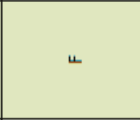 & - & \\
\hline 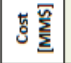 & $\underline{w}$ & $\underline{\underline{z}}$ & $\underline{\underline{z}}$ & $\underline{w}$ & $m$ & $m$ & $\eta$ & $\stackrel{\infty}{\circ}$ & $m$ & \% & $\stackrel{\circ}{\circ}$ & - & $\underline{\underline{z}}$ & 乌े & $\stackrel{\circ}{\circ}$ & $\stackrel{n}{\stackrel{n}{0}}$ & 옹 & $\underline{w}$ & $\underline{w}$ & $\stackrel{\infty}{\infty}$ \\
\hline 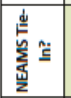 & z & $>$ & $>$ & $>$ & $>$ & $z$ & $>$ & z & $>$ & $z$ & $z$ & z & $z$ & z & $z$ & $z$ & z & $>$ & $>$ & $>$ \\
\hline 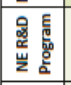 & & & & & \begin{tabular}{|l|} 
\\
$\sum_{3}^{0}$ \\
$\underline{z}$
\end{tabular} & & & & 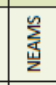 & & & & & & & & & & & \\
\hline 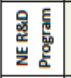 & 妾 & 这 & 离主 & $\breve{\varkappa}$ & $\frac{\dot{\mathrm{u}}}{2} \mathrm{E}$ & $\frac{\dot{s}}{z}$ & $\frac{\sum_{3}^{n}}{\underline{m}}$ & 妾 & 庹已 & 宸 & u & $u$ & 妾e & $u$ & u & u & u & $\sum_{\frac{1}{2}}^{n}$ & $\begin{array}{l}\sum_{x}^{n} \\
\frac{n}{2}\end{array}$ & 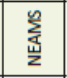 \\
\hline 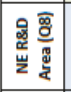 & & & & & $\dddot{\underline{ }}$ & & & & 奠 & E & 岁 & 前 & 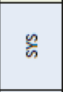 & 盖 & & & & & $\frac{5}{5}$ & \\
\hline 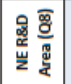 & $\Sigma$ & $\Sigma$ & 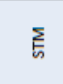 & E & $\frac{\vec{r}}{\underline{z}}$ & $\underline{\underline{\underline{\underline{ }}}}$ & 呟 & 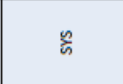 & $\underline{\underline{u}}$ & $\frac{\vec{z}}{\mathbf{z}}$ & $\frac{\vec{d}}{2}$ & 岂 & $\frac{\pi}{2}$ & $\frac{\vec{u}}{z}$ & $\frac{\vec{r}}{2}$ & $\frac{\vec{\mu}}{2}$ & $\frac{\vec{u}}{2}$ & $\overrightarrow{\frac{\vec{z}}{2}}$ & 变 & $\frac{\vec{d}}{2}$ \\
\hline 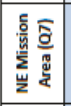 & & & & & & & & & & & & & & & & & & & & $m$ \\
\hline 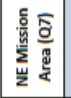 & $\sim$ & $\sim$ & N & $\sim$ & $m$ & + & + & $\sigma$ & + & + & $m$ & $m$ & + & $m$ & $m$ & $m$ & $m$ & + & + & + \\
\hline 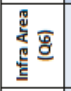 & & & & & 兽 & & 좀 & & & & & & & & & & & & & 兽 \\
\hline 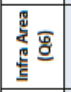 & & & $\vec{\varpi}$ & ఫ్జ & $\frac{z}{4}$ & & $\frac{z}{4}$ & & $\underline{\underline{g}}$ & & & $\frac{z}{4}$ & & 䓪 & 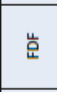 & 萨 & 岂 & & & $\underline{\underline{\underline{0}}}$ \\
\hline 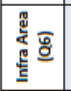 & $\frac{\mathrm{d}}{\mathrm{B}}$ & $\frac{x}{\frac{w_{0}}{2}}$ & $\frac{.0}{\frac{1}{2}}$ & $\frac{.0 .}{\frac{10}{2}}$ & 좀 & $\frac{z}{4}$ & 浐 & × & $\frac{z}{\alpha}$ & 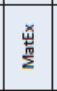 & & 弟 & $\stackrel{\ddot{\underline{x}}}{\underline{x}}$ & $\frac{x}{\frac{1}{2}}$ & 䠢 & $\frac{.0 .}{\frac{1}{2}}$ & 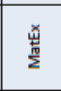 & & & 㟧 \\
\hline 旁 & 展 & 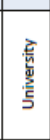 & 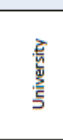 & 喜 & 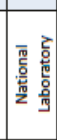 & , & & 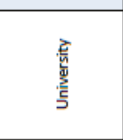 & 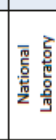 & 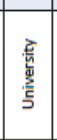 & 喜 & 喜 & 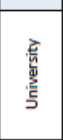 & 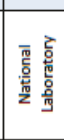 & 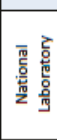 & 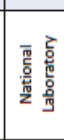 & 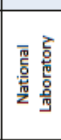 & 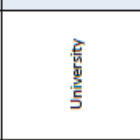 & $\begin{array}{l}\text { 意 } \\
\text { 竞 }\end{array}$ & 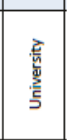 \\
\hline 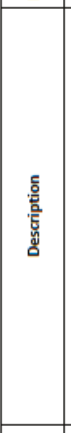 & 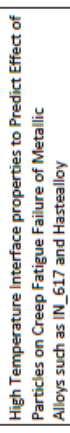 & 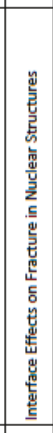 & 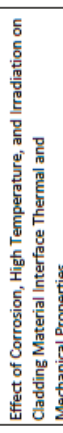 & 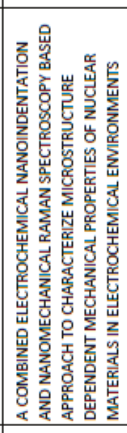 & 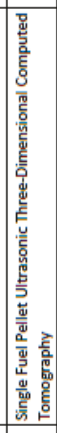 & 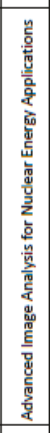 & & 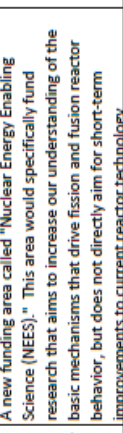 & 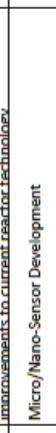 & 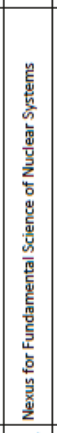 & 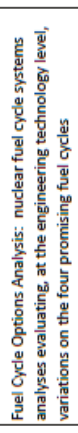 & 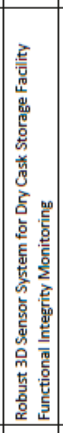 & 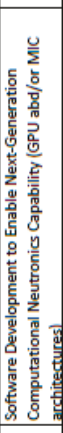 & 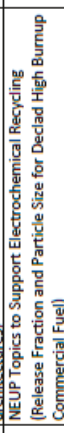 & 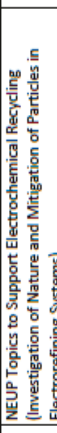 & 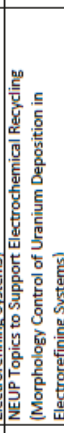 & 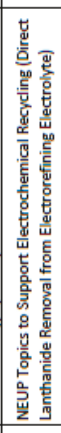 & 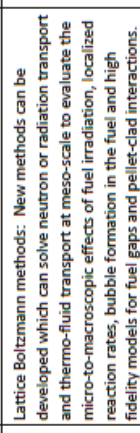 & 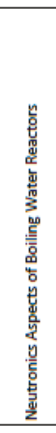 & 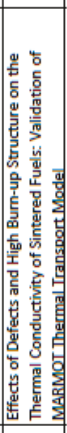 \\
\hline 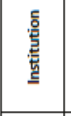 & 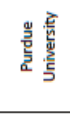 & 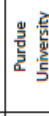 & 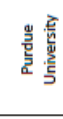 & 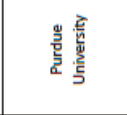 & 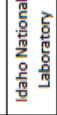 & & & 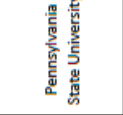 & & & 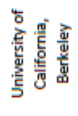 & 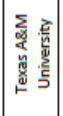 & & 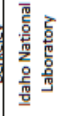 & 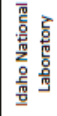 & 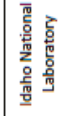 & 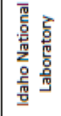 & 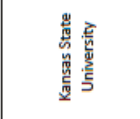 & & 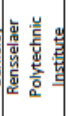 \\
\hline 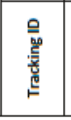 & 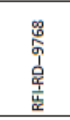 & 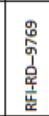 & 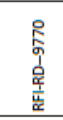 & 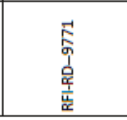 & 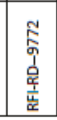 & 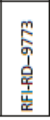 & \begin{tabular}{|l|l} 
\\
弆 \\
童
\end{tabular} & 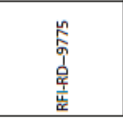 & 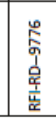 & 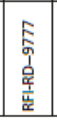 & 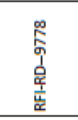 & 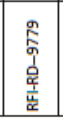 & 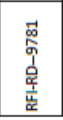 & 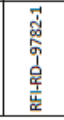 & 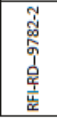 & 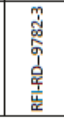 & 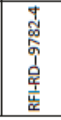 & 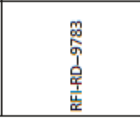 & 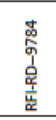 & 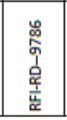 \\
\hline
\end{tabular}




\begin{tabular}{|c|c|c|c|c|c|c|c|c|c|c|c|c|c|c|c|c|c|c|c|c|c|c|c|}
\hline 蒙 & & & & & & & & & & & & & & & & & & & & & & $\underline{\underline{\underline{\alpha}}}$ & \\
\hline 总 & & & ลิ & \%̆ & $\stackrel{m}{0}$ & & & & & & & సิ & F & & 몽 & & & Aิ & & & Aิ & 복 & 吕 \\
\hline 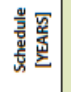 & एu & 荘 & $m$ & $m$ & $m$ & 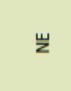 & $\underline{\underline{z}}$ & 岁 & $\underline{\underline{z}}$ & एu & 宸 & m & $m$ & $\sim$ & $m$ & $m$ & m & $m$ & $m$ & $m$ & $m$ & $m$ & $m$ \\
\hline 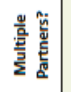 & $z$ & $z$ & $>$ & $>$ & $>$ & $>$ & $>$ & $>$ & $>$ & $>$ & $=$ & $>$ & $>$ & $>$ & $>$ & $>$ & $>$ & $z$ & $>$ & $>$ & $>$ & $>$ & $>$ \\
\hline 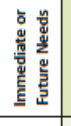 & - & - & - & - & - & - & - & - & - & - & - & - & $u$ & 4 & - & - & - & 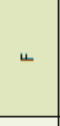 & 4 & ${ }^{u}$ & ${ }^{4}$ & 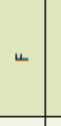 & - \\
\hline 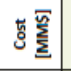 & एu & 㟧 & $\stackrel{\infty}{\circ}$ & $\vec{H}$ & ১̊ & 岁 & $\underline{z}$ & $\underline{z}$ & $\underline{z}$ & w & 宸 & : & 望 & $\sim$ & $\bumpeq$ & $\begin{array}{l}\infty \\
0 \\
\vdots \\
0 \\
0\end{array}$ & $\begin{array}{c}\infty \\
\vdots \\
0 \\
0\end{array}$ & $\stackrel{\infty}{\circ}$ & $\overrightarrow{\dot{\omega}}$ & 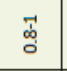 & $\stackrel{\infty}{\circ}$ & $y$ & $\eta$ \\
\hline 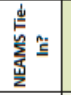 & $z$ & $>$ & $z$ & $z$ & $z$ & $>$ & $z$ & $z$ & $z$ & $z$ & $z$ & $z$ & $>$ & $z$ & $z$ & $z$ & $z$ & $z$ & $z$ & $z$ & $z$ & $z$ & $z$ \\
\hline 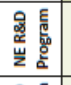 & & & & & & & & & & & & $\ddot{\propto}$ & 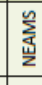 & & & & & $\ddot{x}$ & & & & & \\
\hline 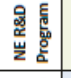 & 咅 & $u$ & $\breve{u}$ & 嵩 & $u$ & 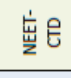 & u & $\breve{u}$ & 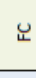 & $\breve{u}$ & 起 & u & $u$ & $u$ & 愛E & $u$ & $u$ & $u$ & 离导 & 宸 & 芘 & 妾 $\mathrm{E}$ & 立 \\
\hline 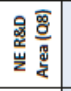 & 益 & & $\frac{\vec{j}}{2}$ & 㒸 & $\frac{\vec{\mu}}{2}$ & $\underline{\xi}$ & & & & & $\underline{\xi}$ & & & 差 & & 5 & $\underline{5}$ & 号 & $\stackrel{\underline{g}}{\underline{m}}$ & $\underline{\underline{g}}$ & & & \\
\hline 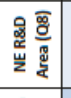 & $\underline{\underline{u}}$ & $\frac{\vec{u}}{2}$ & 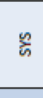 & $\dddot{\underline{g}}$ & 5 & $\frac{\vec{u}}{2}$ & $\overrightarrow{\vec{t}}$ & $\frac{\vec{u}}{z}$ & $\frac{\vec{y}}{2}$ & $\frac{\vec{y}}{2}$ & $\frac{\vec{u}}{z}$ & 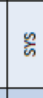 & $\overrightarrow{\frac{\pi}{z}}$ & 妾 & 品 & $\frac{\vec{r}}{2}$ & $\frac{\vec{u}}{2}$ & 品 & $\sum$ & $\frac{5}{4}$ & 立 & 㐫 & 立 \\
\hline 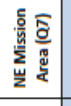 & & & & & & & & & & & & & + & & & & & N & & & & & \\
\hline 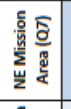 & + & $m$ & $m$ & + & $m$ & + & $m$ & $m$ & $m$ & $m$ & + & + & $m$ & $m$ & + & $m$ & m & $m$ & + & $\sigma$ & + & + & + \\
\hline 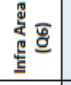 & & 䍜 & & & & & & & & & 曾 & & $\frac{0}{3}$ & & & 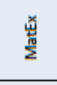 & & & & & & & \\
\hline 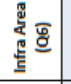 & $\stackrel{u}{\underline{\underline{u}}}$ & 조 & & & & & 噌 & 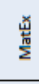 & $\frac{.0 .0}{2}$ & $\frac{\text { 噌 }}{2}$ & 岕 & & $\underline{\underline{\mathbf{B}}}$ & 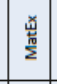 & & $\underline{\underline{\underline{0}}}$ & 宸 & & $\sum$ & $\sum$ & 容 & & \\
\hline 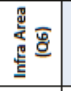 & $\frac{z}{\alpha}$ & 崌 & & $\dddot{\underline{\underline{ }}}$ & & $\frac{x}{\frac{x}{2}}$ & 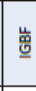 & ఫ્ય & $\vec{\Phi}$ & x & Х & & 总 & 崌 & 菫 & క & $\vec{\varpi}$ & & $\frac{z}{\alpha}$ & $\frac{z}{4}$ & × & & 崖 \\
\hline 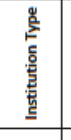 & 居 & 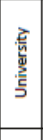 & 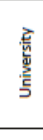 & 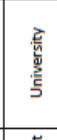 & 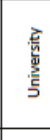 & $\begin{array}{l}\text { 意 } \\
\frac{u_{5}}{5}\end{array}$ & 居 & 总 & 总 & 聪 & 喜 & 意 & 毫 & $\mid \begin{array}{l}\text { 縍 } \\
\underline{\underline{n}}\end{array}$ & $\begin{array}{l}\text { 意 } \\
\text { 鄫 }\end{array}$ & $\begin{array}{l}\text { 离 } \\
\text { 鄫 }\end{array}$ & $\begin{array}{l}\text { 离 } \\
\text { 鄫 }\end{array}$ & $\begin{array}{l}\text { 意 } \\
\text { 旁 }\end{array}$ & 喜 & $\begin{array}{l}\text { 意 } \\
\text { 蚝 }\end{array}$ & 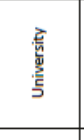 & $\begin{array}{l}\text { 意 } \\
\text { 㟯 }\end{array}$ & $\begin{array}{l}\text { 咅 } \\
\frac{2}{5}\end{array}$ \\
\hline 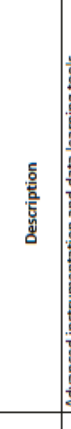 & 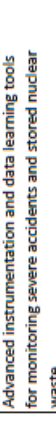 & & & 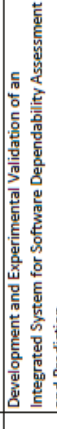 & 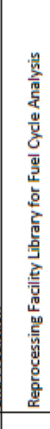 & 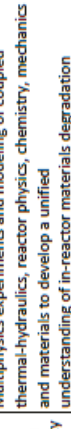 & & 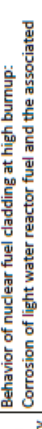 & 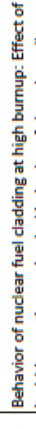 & & & 总 & 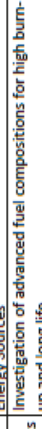 & 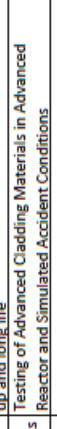 & & 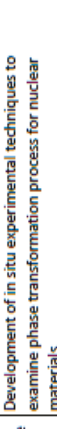 & 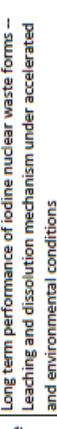 & 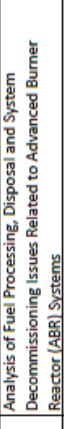 & 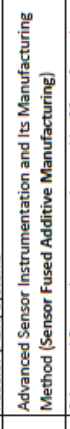 & 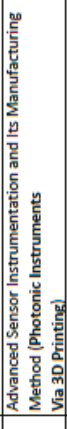 & 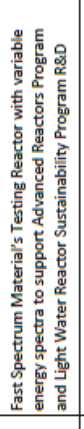 & 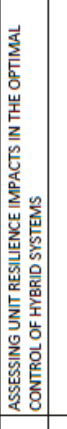 & 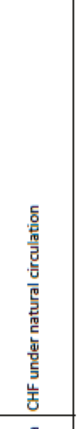 \\
\hline 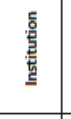 & 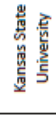 & & & & & 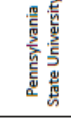 & & & & & & & & & & 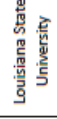 & 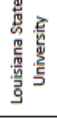 & 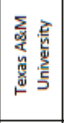 & 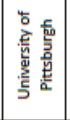 & 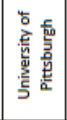 & 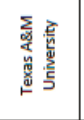 & 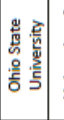 & 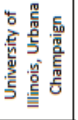 \\
\hline 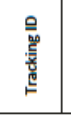 & 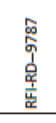 & 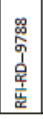 & s. & 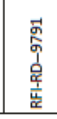 & 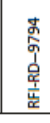 & 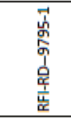 & 鄫 & 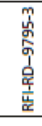 & $\begin{array}{l}\text { 品 } \\
\text { के }\end{array}$ & 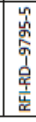 & 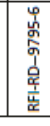 & 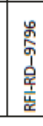 & 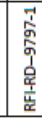 & 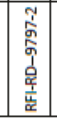 & 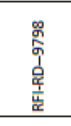 & 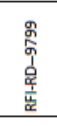 & 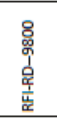 & 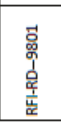 & 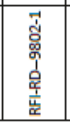 & 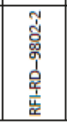 & 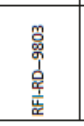 & 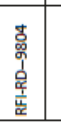 & 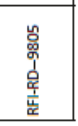 \\
\hline
\end{tabular}


Appendix 2: Request for Information DE-SOL-0008246

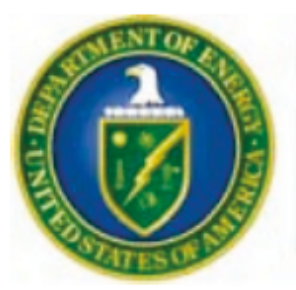

U.S. DEPARTMENT OF

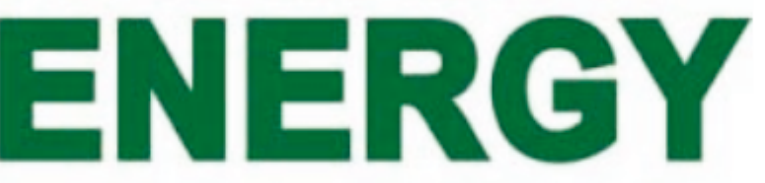

\section{REQUEST FOR INFORMATION \\ DE-SOL-0008246}

University, National Laboratory, Industry, and International Entities Input to the Office of Nuclear Energy's Competitive Research and Development Work Scope Development April 13, 2015

Office of Nuclear Energy

Office of Innovative Nuclear Research 


\section{Table of Contents}

1 Introduction

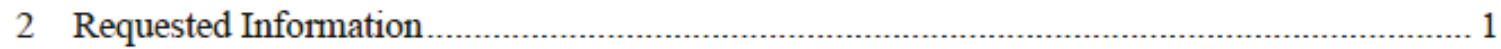

2.1 Cover Page

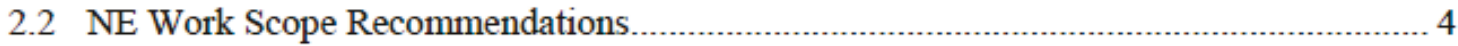

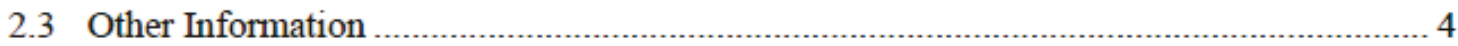

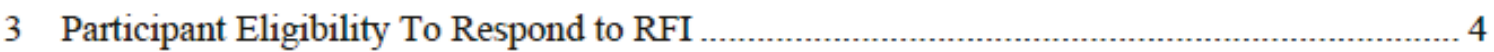

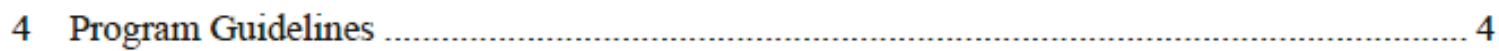

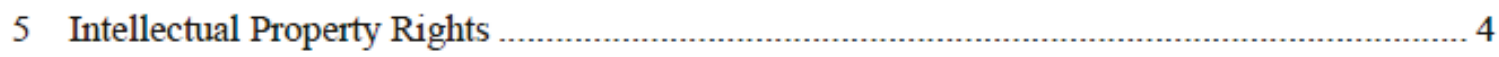

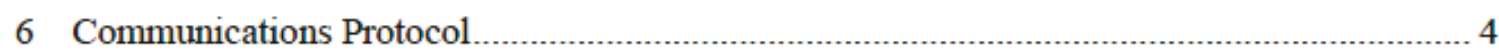

7 Schedule

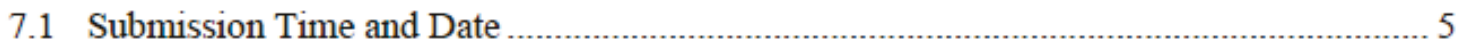

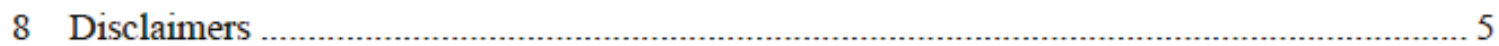




\section{Introduction}

The primary mission of the Office of Nuclear Energy is to advance nuclear power as a resource capable of meeting the Nation's energy, environmental, and national security needs by resolving technical, cost, safety, proliferation resistance, and security barriers through research, development, and demonstration as appropriate.

NE's program is guided by the four research objectives detailed in its Nuclear Energy Research and Development Roadmap:

- Develop technologies and other solutions that can improve the reliability, sustain the safety, and extend the life of current reactors.

- Develop improvements in the affordability of new reactors to enable nuclear energy to help meet the Administration's energy security and climate change goals.

- Develop sustainable fuel cycles.

- Understand and minimize the risks of nuclear proliferation and terrorism.

NE strives to promote integrated and collaborative research conducted by national laboratory, university, industry, and international partners under the direction of NE's programs. NE funds research activities through both competitive and direct mechanisms, as required to best meet the needs of NE. This approach ensures a balanced R\&D portfolio and encourages new nuclear power deployment with creative solutions to the universe of nuclear energy challenges. The competitive portion of NE's R\&D portfolio is executed through the Nuclear Energy University Program (NEUP) and Nuclear Energy Enabling Technologies Crosscutting Technology Development (NEET CTD). NEUP utilizes up to 20 percent of funds appropriated to NE's R\&D program for university-based infrastructure support and R\&D in key NE program-related areas: Fuel Cycle Research and Development (FCR\&D), Reactor Concepts Research, Development and Demonstration (RCRD\&D), and Nuclear Energy Advanced Modeling and Simulation (NEAMS). NEET CTD supports national laboratory, university and industry led crosscutting research.

\section{Requested Information}

1) DOE is seeking ideas in the areas of research, information, comments, feedback, and recommendations from interested parties for future work scopes for the major NE-funded research programs. This input may lead to a more robust $R \& D$ program that reflects the communities' ideas and could identify new work scopes. Although the focus of this Request For Information (RFI) is to obtain input to and identify potential new work scopes for future Funding Opportunity Announcements in the areas listed below, DOE also seeks input on creative, innovative and transformative work that aligns with NE's mission.

Fuel Cycle Research and Development (FC R\&D) Program. The mission of the FC R\&D program is to develop used nuclear fuel management strategies and technologies to support 
meeting the federal government responsibility to manage and dispose of the Nation's commercial used nuclear fuel and high-level waste and to develop sustainable fuel cycle technologies and options that improve resource utilization and energy generation, reduce waste generation, enhance safety, and limit proliferation risk.

The program vision is that by mid-century, strategies and technologies for the safe, long-term management and eventual disposal of U.S. commercial used nuclear fuel and any associated nuclear wastes have been fully implemented. Additionally, it is desired that advanced nuclear fuel and fuel cycle technologies that enhance the accident tolerance of light-water reactors and enable sustainable fuel cycles are demonstrated and deployed. Together, these technologies and solutions support the enhanced availability, affordability, safety, and security of nucleargenerated electricity in the United States.

Current challenges include the development of high burnup fuel and cladding materials to withstand irradiation for longer periods of time with improved accident tolerance; development of simplified materials recovery technologies, waste management (including storage, transportation, and disposal), and proliferation risk reduction methods; and development of processes and tools to evaluate sustainable fuel cycle system options and to effectively communicate the results of the evaluation to stakeholders.

Reactor Concepts Research, Development and Demonstration (RC RD\&D) Program. The mission of the RC RD\&D program is to develop new and advanced reactor designs and technologies that broaden the applicability, improve the competitiveness, and ensure the lasting contribution toward meeting our Nation's energy and environmental challenges. Research activities are designed to address the technical, cost, safety, and security issues associated with various reactor concepts. The four technical areas are Light Water Reactor Sustainability (LWRS), Small Modular Reactors (SMR), Advanced (Non-Light Water) Reactor Concepts (ARC) and Advanced Small Modular Reactors (Adv SMRs). In addition, R\&D for the manufacturing of radioisotope power systems for national security and space exploration missions is supported through the Space and Defense Infrastructure Program.

Nuclear Energy Advanced Modeling and Simulation (NEAMS) Program. The mission of the NEAMS program is to create modern computer simulation codes and methods that give the user state-of-the-art physics models that can take advantage of powerful multi-processing computers in order to better understand the behavior of nuclear reactor and fuel systems during normal operations and/or transient events. In particular, NEAMS is aimed at creating an advanced mechanistic toolkit that is applicable to a wide range of reactor designs for use by industry, academia, and the national laboratories. The NEAMS Toolkit will help engineers and scientists form new insights into the safety and economics of current and next generation reactor and fuel systems. It will provide much higher fidelity than current methods and incorporate well-defined and validated prediction capabilities.

This will be achieved by employing advanced software environments and modern highperformance computers to create a set of engineering-level codes in which fuels and materials continuum properties are informed by first-principles modeling of materials at the atomistic and meso-scale. A set of simulation tools will be developed that promote interoperability of codes with respect to spatial meshing, materials and fuels models, and achieve a common 
"look and feel" for setting up problems and displaying results. The toolset to be developed aims to achieve scalability in terms of computing power and the types and couplings of the physics that dominates the system behavior.

The Department of Energy's (DOE) Office of Nuclear Energy (NE) conducts crosscutting nuclear energy research and development $(R \& D)$ and associated infrastructure support activities to develop innovative technologies that offer the promise of dramatically improved performance for advanced reactors and fuel cycle concepts while maximizing the impact of DOE resources.

Nuclear Energy Enabling Technologies (NEET) Crosscutting Technology Development (CTD). NEET CTD competitively awards high-priority R\&D to universities, national laboratories, and industry, leading to the development of innovative solutions to unique and crosscutting nuclear energy challenges. The subprogram works in close coordination with NE's other R\&D programs to ensure that developed technologies and capabilities address critical technology gaps as part of an integrated solution offering the potential of revolutionary improvement in safety, performance, reliability, economics, and proliferation risk reduction. Additionally, NEET CTD strategically invests in competitive, nuclear energy-related infrastructure enhancement at national laboratories; ensuring researchers have access to stateof-the-art R\&D capabilities. The capabilities developed through NEET CTD advance the state of nuclear technology, improving its competitiveness, and promoting continued contribution to meeting our Nation's energy and environmental challenges.

Replies to this request should follow the general organization of Section 2 of this RFI and information should be as succinct as possible. Respondents are encouraged to provide input on any areas of interest of this RFI.

\subsection{Cover Page}

Responses shall include a cover page containing the following information:

- RFI title

- Names, phone numbers, and e-mail addresses for the principal points of contact

- Company or affiliate name and address

- Date of submittal 


\subsection{NE Work Scope Recommendations}

Clearly define your proposed work scope, and how it relates to any part of NE's mission described in this RFI. Describe any defined goals in achieving the desired outcomes, along with appropriate metrics to assess how well those goals have been achieved.

2) What would be the estimated cost of the work scope?

3) Would the work scope be more focused on immediate NE program needs, or more creative, innovative and transformative?

4) Would the work scope require multiple partners?

5) What would be a reasonable schedule duration and key milestones?

\subsection{Other Information}

Provide any other relevant information you feel is important not otherwise already covered including comments or suggestions.

\section{Participant Eligibility to Respond to RFI}

Information is being sought from educational institutions, National Laboratories, utilities, private industry, international entities, and any other interested party.

\section{Program Guidelines}

This market research request is done under the Federal Acquisition Regulation (FAR), Parts $10-$ Market Research and FAR subpart 15.201(e) - Requests for Information.

\section{Intellectual Property Rights}

Participants are advised that their RFI response package should be submitted without any restrictive markings. However, if restrictions are required in order to fully explain a response, the participant is responsible to mark the cover page and any and all submittal documents appropriately. Respondents are strongly discouraged from placing any restrictive markings on submissions as they may limit DOE's ability to use the submitted information.

\section{Communications Protocol}

Responses must be submitted through www.NEUP.gov to be considered. You must create an account to access the submission site. Submit electronic submissions through the "Applications" function at www.NEUP.gov. If you have problems completing the registration process or submitting your response, call 208-526-1507 or send an email to NEUP@inl.gov. 
Participants are advised that any indication of interest, in the affirmative, is not meant to imply nor in any way impart an obligation on the part of the Government that an award will be forthcoming for the offered work or project.

\section{Schedule}

\subsection{Submission Time and Date}

The DOE will continually accept packages in response to this RFI No. DE-SOL-0008246. However to be considered for the 2016 grant opportunities a response will be required no later than 8:00 p.m. ET, June 19, 2015.

This announcement does not impose any obligation on the Government nor does it signify any intent for a contract or other form of award.

\section{Disclaimers}

a. DOE does not plan to send individual acknowledgements or replies to respondents to the RFI. However, DOE may conduct one-on-one meetings with entities that respond to this request if clarification or additional information is required to improve the DOE's understanding of the comments provided. If DOE decides to hold one-on-one meetings, applicable interested parties will be contacted. The decision to meet with a company one-on-one has no bearing on the worthiness of its RFI submittal or on any future offerings.

b. This is a request for information only. It has no direct relation to other DOE Funding Opportunity Announcements or solicitations. DOE does not presently intend to solicit or award any kind of contract or financial assistance award; this RFI is issued only with the intent of obtaining information.

c. Any response to this RFI is voluntary and does not commit to Government to any expense or obligation. This request does not impose any obligation on the Government or signify a firm intention to enter into a contract. No costs associated with responding to this RFI or participating in any subsequent meetings will be borne by the Government.

d. DOE does not intend to publish the results of the responses to this RFI. 